\title{
Finitely Correlated States on Quantum Spin Chains
}

\author{
M. Fannes ${ }^{1,2}$, B. Nachtergaele ${ }^{3,4}$, and R. F. Werner ${ }^{5}$ \\ ${ }^{1}$ Inst. Theor. Fysica, Universiteit Leuven, Leuven, Belgium \\ 2 Bevoegdverklaard Navorser, N.F.W.O. Belgium \\ 3 Depto de Física, Universidad de Chile, Casilla 487-3, Santiago de Chile \\ ${ }^{4}$ Onderzoeker I.I.K.W. Belgium, on leave from Universiteit Leuven, Belgium \\ 5 Dublin Institute for Advanced Studies, 10 Burlington Road, Dublin 4, Ireland, On leave from \\ Universität Osnabrück, FRG
}

Received July 12, 1990; in revised form June 3, 1991

\begin{abstract}
We study a construction that yields a class of translation invariant states on quantum spin chains, characterized by the property that the correlations across any bond can be modeled on a finite-dimensional vector space. These states can be considered as generalized valence bond states, and they are dense in the set of all translation invariant states. We develop a complete theory of the ergodic decomposition of such states, including the decomposition into periodic "Néel ordered" states. The ergodic components have exponential decay of correlations. All states considered can be obtained as "local functions" of states of a special kind, so-called "purely generated states," which are shown to be ground states for suitably chosen finite range VBS interactions. We show that all these generalized VBS models have a spectral gap. Our theory does not require symmetry of the state with respect to a local gauge group. In particular we illustrate our results with a one-parameter family of examples which are not isotropic except for one special case. This isotropic model coincides with the one-dimensional antiferromagnet, recently studied by Affleck, Kennedy, Lieb, and Tasaki.
\end{abstract}

\section{Introduction}

Determining ground state properties of quantum spin systems on a lattice is often a hard problem, and is certainly much more complex than the corresponding problem in classical statistical mechanics. One reason for this difference is that in a classical theory the energy of a state can be minimized locally, by fixing the state on the boundary $\partial \Lambda$ of a finite region $\Lambda$, and finding the local state in $\Lambda$ of minimal energy with the prescribed marginals on the sites in $\partial \Lambda$. This procedure breaks down in a quantum system, because the local state obtained in this way, and the state outside $\Lambda$ may fail to have a common extension [65]. A closely related point 
is the following. Ground states can be expected to be pure in both cases, and in the classical case this implies the purity of the local restrictions. In particular, translation invariant pure states in a classical lattice system have a completely trivial structure. For an Ising system, for example, there are just two such states, either all spins are up, or all are down. In quantum mechanics the restriction of a pure state is usually not pure, and consequently a translation invariant pure state may have a rich structure of long range correlations. Thus in a quantum spin system it is not trivial to establish rigorously properties such as the uniqueness or degeneracy of the ground state (spontaneous symmetry breaking), the decay law of correlation functions, and the spectrum of low-lying excitations in the ground state (occurrence of a spectral gap above the ground level or not). The aim of this paper is to present and study a class of states, and related Hamiltonians, for which such questions can be answered explicitly.

The above mentioned extension problem for quantum states is trivial when one is dealing with product states, and these are the states arising as the ground states of purely ferromagnetic models. Models of antiferromagnetism gained new interest in recent years mostly because of their relation to high- $T_{c}$ superconductivity (see e.g. [7]), and it is no surprise that a lot of the complexity of quantum ground states has turned up in the study of such models. The uniqueness of the finite volume ground state for a large class of models, including the standard nearest neighbor isotropic Heisenberg antiferromagnets on any finite bipartite lattice, was decided by a beautiful theorem of Lieb and Mattis [52]. In [51] it was shown that the Heisenberg model on an infinite chain does not exhibit a spectral gap above the ground state. An interesting form of nonuniqueness of ground states is the occurrence of Néel order in translation and rotation invariant models. Clearly, one has to consider systems in the thermodynamic limit in order to get relevant examples of symmetry breaking. This phenomenon was demonstrated in certain cases by Dyson, Lieb and Simon in [25], and in more recent extensions of this work (e.g. [47]). A very stimulating conjecture was made by Haldane [38]. He predicted that the behavior of the ground states of one-dimensional antiferromagnetic nearest neighbor interactions would depend qualitatively on the fact whether the value of the spin $s$ is integer or half-integer. For a discussion and a proof of part of the conjecture see [4]. In the case of one-dimensional models several Hamiltonians are known, which can be solved exactly by the Bethe Ansatz $[14,41,63,11]$ or with the use of Temperly-Lieb algebras or Yang-Baxter type methods $[12,13,49]$, and which have been inspiring examples in many branches of theoretical physics. For these models the ground state energy, and the absence or existence of a gap usually can be obtained. However, in all models mentioned so far the determination of the correlation functions presents considerable problems.

Correlation functions are relatively simple to obtain for another class of models, for which the ground states can be constructed exactly $[48,5,9,22,43]$. They are called VBS models, because of the Valence Bond structure of their ground states. After suitable generalization one finds that the much older Majumdar-Ghosh model $[53,54,5]$, has the same structure, although the ground states are especially simple there. The states, which we investigate are generalizations of valence bond states, and before we sketch the main results of our paper, it may be in order to recall the paradigm of such a state, namely a state on the spin 1 chain studied in 
detail in [5]. In a certain sense it is the simplest nontrivial state of the class we study.

Affleck, Kennedy, Lieb, and Tasaki [5] consider the Hamiltonian

$$
H=\sum_{i}\left\{\frac{1}{2} \mathbf{S}_{i} \cdot \mathbf{S}_{i+1}+\frac{1}{6}\left(\mathbf{S}_{i} \cdot \mathbf{S}_{i+1}\right)^{2}+\frac{1}{3}\right\},
$$

where $\mathbf{S}_{i}$ denotes the generators of the irreducible spin 1 representation of $S U(2)$, which lives in the one-site algebra at site $i$. The expression in braces is nothing but the projection onto the spin 2 subspace in the decomposition of the tensor product of the two representations at sites $i$ and $(i+1)$. The basic results of [5] concerning this Hamiltonian are that it has a unique ground state, which can be given by a fairly explicit "valence bond" construction. It has exponentially decaying correlation functions, which can be computed explicitly. Moreover, there is a spectral gap above the ground state. It is remarkable that this ground state does minimize the energy locally, i.e. each term in the above sum is positive semidefinite, and has zero expectation in the ground state. The construction of the state involves a contraction scheme with respect to indices of certain representations of $S U(2)$, which can also be generalized to some other groups [3]. The $S U(2)$ valence bond states can also be expressed rather effectively in terms of homogeneous polynomials in two variables $[9,46,50]$. In all these studies the presence of a gauge symmetry group for the state under consideration plays a decisive rôle. It is therefore not clear a priori whether models with the properties proven by [5] are singular occurrences, or simply the gauge invariant examples in a larger class. We will show in this paper that the latter is the case. To this end we use an abstract definition of (generalized) valence bond states, which does not involve any symmetry group. The ground state of the above model is thereby embedded into a 19-dimensional manifold of valence bond states, each of which is the unique ground state of a certain class of finite range Hamiltonians. Of each such state we will prove essentially all the results obtained in [5] for the special example. All these results will be worked out in detail for the following one-parameter deformation of the AKLT model:

$$
\begin{aligned}
H= & \sum_{i}\left\{\frac{1}{2} \mathbf{S}_{i} \cdot \mathbf{S}_{i+1}+\left(\frac{1}{6}+\frac{1}{3} \eta(t)\right)\left(\mathbf{S}_{i} \cdot \mathbf{S}_{i+1}\right)^{2}+\left(\frac{1}{3}-\frac{4}{3} \eta(t)\right)+\eta(t)\left(S_{i}^{z}\right)^{2}\right. \\
& \left.-\frac{1-t}{1+t} \eta(t)\left(S_{i}^{z} S_{i+1}^{z}\right)^{2}+\frac{1-t}{1+t} \frac{\eta(t)}{2}\left\{S_{i}^{z} S_{i+1}^{z}, \mathbf{S}_{i} \cdot \mathbf{S}_{i+1}\right\}\right\},
\end{aligned}
$$

where $t \in[0,1]$ and $\eta(t)=\left(3 t^{2}+2 t-1\right) /\left(6 t^{2}-4 t+2\right)$. The AKLT model corresponds to $t=1 / 3(\eta=0)$.

In a quite different context the construction we use was suggested in $[1,2]$. It emphasizes the rôle of a family of operators, which are reminiscent of transfer matrices. Although the notion of a transfer matrix is usually limited to the context of classical systems a generalization to quantum spin chains has been introduced by [8] in order to prove uniqueness and analyticity properties of Gibbs states for finite range interactions. It should be noted that in contrast with the case of classical spin systems, such a transfer matrix essentially lives on an infinite-dimensional space. Unlike the approach of [8], the fundamental difference between the quantum 
and the classical situation in our approach lies in the positivity properties of the transfer matrix, rather than in the structure of the space it lives on. In specific examples of VBS models the utility of transfer matrix-like objects was also realized by other authors $[35,9,43,44]$.

As the essential feature characterizing the states obtainable by our construction we single out the property that the correlations across any bond of the chain can be modeled on a finite-dimensional vector space. A subclass of states with this property, called $C^{*}$-finitely correlated states is then shown to be identical with the class of valence bond states according to our abstract definition (Proposition 2.7). Our aim is to give a general theory of this class of translation invariant states on spin chains. Whatever the merits of the valence bond picture on lattices of higher dimension, we found the transfer matrix point of view the more helpful representation on one-dimensional lattices, and therefore made it the starting point of our investigation. A major advantage, both for practical computations and for general considerations, is that the computation of correlation functions in our approach reduces to obtaining the spectral properties of a finite dimensional matrix. In particular, all these states have exponential decay of correlations. For example, in the case of the state on the spin 1 chain as studied by [5] the valence bond picture suggested a fairly involved diagrammatic technique to obtain the correlation functions [5], whereas in our approach the computation reduces to evaluating one matrix element of a diagonal $4 \times 4$-matrix.

We now give a more detailed overview of the results presented in the different sections of this paper, without, however, entering into the technicalities. In order to illustrate the different stages of our analysis we will carry through the paper the one-parameter family of examples (1.2). Occasionally we will also consider the Majumdar-Ghosh model $[53,54]$ and the Heisenberg ferromagnet.

Section 2. Finitely Correlated States. Throughout the paper we are concerned with translation invariant states on the chain algebra $\mathscr{A}_{\mathbb{Z}} \equiv \bigotimes_{i \in \mathbb{Z}} \mathscr{A}_{i}$, where $\mathscr{A}_{i}$ denotes a copy of a fixed $C^{*}$-algebra $\mathscr{A}^{\text {"at }}$ site $i$." Finitely correlated states on $\mathscr{A}_{\mathbb{Z}}$ are defined by the property that the correlations across any bond can be modeled on a finite-dimensional vector space $\mathscr{B}$. We show that the state can then be reconstructed from a map $\mathbb{E}: \mathscr{A} \otimes \mathscr{B} \rightarrow \mathscr{B}$, and two elements $e \in \mathscr{B}, \rho \in \mathscr{B} *$. For most of the paper we specialize to the case of " $C^{*}$-finitely correlated states," for which $\mathscr{B}$ is a finite-dimensional $C^{*}$-algebra, and $\mathbb{E}, e, \rho$ are (completely) positive. The class of $C^{*}$-finitely correlated states is shown to be a ${ }^{*}$ weakly dense convex subset of the set of translation invariant states, which is important for the possibility of using these states as trial states in variational computations. We define generalized valence bond states, and show that on spin chains they coincide with the $C^{*}$-finitely correlated states.

Section 3. Ergodic Decompositions. Correlation functions of a $C^{*}$-finitely correlated state are expressed in terms of the powers of the operator $\hat{\mathbb{E}}(B)=\mathbb{E}\left(\mathbb{1}_{\mathscr{A}} \otimes B\right)$ on $\mathscr{B}$. If $e \in \mathscr{B}$ is the only fixed point of $\hat{\mathbb{E}}$ then the state is exponentially clustering, and hence ergodic (i.e. extremal translation invariant). We show that every $C^{*}$-finitely correlated state has a unique convex decomposition into finitely many ergodic $C^{*}$-finitely correlated states. Using a quantum version of the classical 
Perron-Frobenius theory, the breaking of translation invariance, i.e. the decomposition of the given state into periodic components, can be diagnosed from the set of eigenvalues of $\hat{\mathbb{E}}$ with modulus one. All these eigenvalues are necessarily roots of unity, i.e. quasi-periodic behavior is excluded.

Section 4. Dilation Theory and Purely Generated States. We continue the reduction of general $C^{*}$-finitely correlated states to simpler building blocks. In classical probability theory finitely correlated states can be seen as functions of Markov Processes (see Sect. 7.1). In this section we identify a subclass, the "purely generated states," which generate all $C^{*}$-finitely correlated states by 'taking functions.' What is meant by 'taking functions' in the noncommutative context is explained there. The purely generated states are those for which the map $\mathbb{E}$ is "pure," i.e. it cannot be written as the sum of other completely positive maps. Equivalently, $\mathbb{E}(X)=V^{*} X V$ for an isometry $V$ between appropriate Hilbert spaces. The set of pure completely positive maps on a quantum system has a much richer structure than its counterpart in classical probability. This structure is essential in Sects. 4, 5, and 6. In particular, it allows the construction of an abundance of nontrivial pure states. Sections 3 and 4 together amount to the identification of the basic building blocks for all $C^{*}$-finitely correlated states: these are the purely generated states which have no proper decomposition into periodic components, or equivalently, which are exponentially clustering.

Section 5. Ground State Property of Purely Generated States. Here a crucial step for the applications is made. It is shown that each of the basic building blocks identified above, i.e. every purely generated exponentially clustering $C^{*}$-finitely correlated state, is the unique ground state of some translation invariant finite range interaction. The interaction is chosen such that the energy density is equal to the lowest eigenvalue of the interaction operator, i.e. the state minimizes the energy locally. As a by-product, we prove that every purely generated exponentially clustering state is pure, i.e. it cannot be decomposed even into non-translation invariant components, and we also obtain a formula for the (finite) limiting absolute entropy of these states (the entropy density vanishes).

Section 6. The Ground State Energy Gap. Continuing the study of the Hamiltonians introduced in the previous section, it is shown that all these models have a spectral gap immediately above the ground state. The methods presented here are tailored to get a simple proof of the existence of the gap. Although they also allow explicit estimates, these estimates are not optimal. We do not know whether one could hope to derive exact expressions also for the gap, as is possible in the integrable models [12]. A short overview of our technique, stated in valence bond language, was given in [30].

Section 7. Applications. We chose only a few examples to highlight the general structure developed in the main body of the paper. Further applications concerned with entropy properties and finitely correlated states on a tree, will be treated elsewhere $[31,32,33]$.

7.1. Classical Systems. In order to put our results for quantum spin chains into perspective, we briefly review earlier results [28] for the case that all the $C^{*}$-algebras 
appearing in the general construction are abelian. In this case $C^{*}$-finitely correlated states are precisely the functions of Markov processes. A formula for the dynamical entropy (or entropy density) for such a probability measure is given.

7.2. Integrable Systems. In the classical case any Gibbs state for a finite range interaction is $C^{*}$-finitely correlated, and conversely any faithful Markovian measure is a Gibbs state for a well-defined nearest neighbor Hamiltonian [58, 34]. Unfortunately, in spite of the fact that $C^{*}$-finitely correlated states are dense in the translation invariant states (as noted above), this connection fails in the quantum case, even for ground states. As an example we show that the ground states of some integrable half-integer spin chains, treated by Takhtajan [63], are not $C^{*}$-finitely correlated. Although this can undoubtedly also be demonstrated by other methods, we show that it suffices to note that the known exact ground state energy of these models is transcendental, i.e. not algebraic in the coupling constant.

7.3. Gauge Invariant States. As states and models with a given group invariance (acting on each site) certainly are of special importance, we study this situation in more detail. A straightforward construction for states with given symmetry is given. We apply this construction to obtain the well-known integer spin models $[5,9,29]$. By the results of Sect. 6 all these models have a spectral gap. It is also shown how the representation theory of $S U(2)$ can be used to carry out explicit calculations.

Appendix: Matrix order and complete positivity. Here we prove a characterization result for finitely correlated, but not necessarily $C^{*}$-finitely correlated states, and collect the definitions and results about matrix ordered vector spaces needed for this purpose.

\section{Finitely Correlated States}

In this paper we study a class of states on quantum "spin" chains. The observable algebra for a single "spin" is some fixed $C^{*}$-algebra $\mathscr{A}$ with identity $\mathbb{1}_{\mathscr{A}}$. Often this algebra will be finite-dimensional, or more specifically, the algebra $\mathscr{M}_{d}$ of $d \times d$ matrices. For each $n \in \mathbb{Z}$ we consider an isomorphic copy $\mathscr{A}_{\{n\}}$ of $\mathscr{A}$, and define for each finite subset $\Lambda \subset \mathbb{Z}$ the algebra $\mathscr{A}_{\Lambda}=\bigotimes_{x \in \Lambda} \mathscr{A}_{\{x\}}$. Here and below the symbol " $\otimes$ " will always refer to the minimal $C^{*}$-tensor product [62]. For $\mathscr{A}_{\{1, \ldots, n\}}$ we also write $\mathscr{A}^{\otimes n}$. For infinite subsets $\Lambda \subset \mathbb{Z}, \mathscr{A}_{\Lambda}$ is defined as the $C^{*}$-inductive limit of the algebras $\mathscr{A}_{\Lambda^{\prime}}$ with $\Lambda^{\prime} \subset \Lambda$ finite. The identification $\mathscr{A}_{\Lambda^{\prime \prime}} \subseteq \mathscr{A}_{\Lambda^{\prime}}$ for $\Lambda^{\prime \prime} \subset \Lambda$ underlying this limit is by tensoring $A \in \mathscr{A}_{\Lambda^{\prime \prime}}$ with $\bigotimes_{x \in \Lambda^{\prime} \backslash \Lambda^{\prime \prime}} \mathbb{1}_{\mathscr{A}_{\{x\}}}$. The most important example of this is the chain algebra $\mathscr{A}_{\mathbb{Z}}$ itself. The group $\mathbb{Z}$ acts on $\mathscr{A}_{\mathbb{Z}}$ by the translation automorphisms $\alpha_{r}$, taking $\mathscr{A}_{\Lambda}$ into $\mathscr{A}_{\Lambda+r}$. The set of translation invariant states on $\mathscr{A}_{\mathbb{Z}}$ will be denoted by $\mathscr{T}$, or $\mathscr{T}(\mathscr{A})$. By grouping segments of $p$ sites together, we obtain an isomorphism of $\mathscr{A}_{\mathbb{Z}}$ with $\left(\mathscr{A}^{\otimes p}\right)_{\mathbb{Z}}$, identifying $\mathscr{A}_{\{k p, \ldots, k p+p-1\}}$ with $\left(\mathscr{A}^{\otimes p}\right)_{\{k\}}$.

The characteristic property of the class of translation invariant states on $\mathscr{A}_{\mathbb{Z}}$ studied in this paper is described in (1) of the following proposition. 
2.1 Proposition. Let $\mathscr{A}$ be a $C^{*}$-algebra with unit, and let $\omega$ be a translation invariant state on the chain algebra $\mathscr{A}_{\mathbb{Z}}$. Then the following are equivalent:

(1) The set of functionals $\Phi: \mathscr{A}_{\mathbb{N}} \rightarrow \mathbb{C}$ of the form

$$
\Phi\left(A_{1} \otimes \cdots A_{n}\right)=\omega\left(X \otimes A_{1} \otimes \cdots A_{n}\right),
$$

with $X \in \mathscr{A}_{\mathbb{Z} \backslash \mathbf{N}}$ generates a finite-dimensional linear subspace in the dual of $\mathscr{A}_{\mathbf{N}}$.

(2) There are a finite-dimensional vector space $\mathscr{B}$, a linear map $\mathbb{E}: A \in \mathscr{A} \mapsto$ $\mathbb{E}_{A} \in \mathscr{L}(\mathscr{B}, \mathscr{B})$, an element $e \in \mathscr{B}$, and a linear functional $\rho \in \mathscr{B} *$, such that $\rho \circ \mathbb{E}_{\mathbb{1}}=$ $\rho, \mathbb{E}_{\mathbb{1}}(e)=e$, and for $n \in \mathbb{Z}, m \in \mathbb{N}$ and $A_{i} \in \mathscr{A}_{\{i\}} \cong \mathscr{A}$ :

$$
\omega\left(A_{n} \otimes \cdots A_{n+m}\right)=\rho(e)^{-1} \rho \circ \mathbb{E}_{A_{n}} \circ \cdots \circ \mathbb{E}_{A_{n+m}}(e),
$$

where the symbol "०" means composition of maps.

If in (2) $\mathscr{B}$ is chosen as minimal in the sense that

$$
\operatorname{lin}\left\{\mathbb{E}_{A_{1}} \circ \ldots \circ \mathbb{E}_{A_{n}}(e) \mid n \in \mathbb{N}, A_{1}, \ldots, A_{n} \in \mathscr{A}\right\}=\mathscr{B},
$$

and

$$
\operatorname{lin}\left\{\rho \circ \mathbb{E}_{\boldsymbol{A}_{1}} \circ \ldots \circ \mathbb{E}_{A_{n}} \mid n \in \mathbb{N}, A_{1}, \ldots A_{n} \in \mathscr{A}\right\}=\mathscr{B} *,
$$

then $\mathscr{B}, \mathbb{E}, \rho$, and e are determined by $\omega$ up to linear isomorphism.

2.2 Definition. If the equivalent conditions of Proposition 2.1 are satisfied, $\omega$ will be called the finitely correlated state generated $b y(\mathbb{E}, \rho, e)$.

\section{Proof of 2.1}

(1) $\Rightarrow(2)$ : We abbreviate $\mathscr{A}_{\#} \equiv \mathscr{A}_{\{n \mid n \geqq 1\}}$ and $\mathscr{A}_{b} \equiv \mathscr{A}_{\{n \mid n \leqq 0\}}$. On $\mathscr{A}_{\#}$ we consider the equivalence relation $X \sim Y \Leftrightarrow \omega\left(X_{b} \otimes(X-Y)\right)=0$ for all $X_{b} \in \mathscr{A}_{b}$, and an analogous relation on $\mathscr{A}_{b}$. Denote by $\mathscr{B}_{\natural}$ the quotients of $\mathscr{A}_{\natural}$ by these relations and by $\left[X_{\natural}\right] \in \mathscr{B}_{\natural}$ the equivalence class of $X_{\natural} \in \mathscr{A}_{\natural}$, where $\natural$ stands for \# or $b$. Obviously, there is a well defined, nondegenerate bilinear form $\eta: \mathscr{B}_{b} \times \mathscr{B}_{\#} \rightarrow \mathbb{C}$ such that $\eta\left(\left[X_{b}\right],\left[X_{\#}\right]\right)=\omega\left(X_{b} \otimes X_{\#}\right)$. Clearly, $X_{b} \sim X_{b}^{\prime}$ iff $X_{b}^{\prime}$ generate the same functional on $\mathscr{A}_{\#}$, hence (1) implies that $\mathscr{B}_{b}$ is finite-dimensional. Since $\eta$ is nondegenerate, we can identify $\mathscr{B}_{\#}$ with the dual of $\mathscr{B}_{b}$, and we shall take $\mathscr{B}=\mathscr{B}_{\#}$, $e=[\mathbb{1}] \in \mathscr{B}$, and $\rho=[\mathbb{1}] \in \mathscr{B}_{b} \equiv\left(\mathscr{B}_{\#}\right)^{*}$ in $(2)$. Let $\mathbb{E}_{A}\left(\left[X_{\#}\right]\right)=\left[A \otimes X_{\#}\right]$. We have to show that this is well defined, i.e. that $\left[A \otimes X_{\#}\right]=0$, whenever $\left[X_{\#}\right]=0$. But $\left[X_{\#}\right]=0$ implies in particular that $\omega\left(\left(X_{b} \otimes A\right) \otimes X_{\#}\right)=0$ for all $X_{b} \in \mathscr{A}_{\{n \mid n \leqq-1\}}$, and by translation invariance of $\omega$ we also have $\omega\left(X_{b} \otimes\left(A \otimes X_{\#}\right)\right)=0$ for all $X_{b} \in \mathscr{A}_{b}$. The verification of (2.1) is straightforward.

$(2) \Rightarrow(1)$ : Given $\mathscr{B}, e, \rho, \mathbb{E}$ satisfying (2), we define the maps $\mathscr{T}_{\#}: \mathscr{A}_{\#} \rightarrow \mathscr{B}, \mathscr{T}_{b}: \mathscr{A}_{b} \rightarrow \mathscr{B}^{*}$ by

$$
\begin{aligned}
\mathscr{T}_{\#}\left(A_{1} \otimes \cdots A_{n}\right) & =\mathbb{E}_{A_{1}} \circ \cdots \circ \mathbb{E}_{A_{n}}(e), \\
\mathscr{T}_{b}\left(A_{-n} \otimes \cdots A_{0}\right) & =\rho(e)^{-1} \rho \circ \mathbb{E}_{A_{-n}} \circ \cdots \circ \mathbb{E}_{A_{0}} .
\end{aligned}
$$

Then for $X_{b} \in \mathscr{A}_{b}$ we have $\omega\left(X_{b} \otimes X_{\#}\right)=\mathscr{T}_{b}\left(X_{b}\right)\left(\mathscr{T}_{\#}\left(X_{\#}\right)\right)$. Since the range of $\mathscr{T}_{b}$ is in the finite-dimensional space $\mathscr{B}^{*},(1)$ holds. If $\mathscr{B}$ is chosen to be minimal in the sense described, $\mathscr{T}_{b}$ is surjective. Therefore, $\mathscr{T}_{\#}\left(X_{\#}\right)=0$ is equivalent to 
$\omega\left(X_{b} \otimes X_{\#}\right)=0$ for all $X_{b}$, i.e. $X_{\#} \sim 0$. Since $\mathscr{T}_{\#}$ is also surjective, $\left[X_{\#}\right] \mapsto \mathscr{T}_{\#}\left(X_{\#}\right)$ defines a linear isomorphism from $\mathscr{B}_{\#}$ to $\mathscr{B}$.

The proposition gives an explicit formula (2.1) for $\omega$ in terms of the usually much simpler objects $\mathscr{B}, \mathbb{E}, \rho$, and $e$. We would therefore like to turn this formula into a definition of the state $\omega$. It is clear from the structure of this formula, and from the invariance assumptions for $e$ and $\rho$ that the family of functionals on $\mathscr{A}_{\{n, \ldots n+m\}}$ defined by (2.1) is consistent with the injections $\mathscr{A}_{\Lambda^{\prime \prime}} \hookrightarrow \mathscr{A}_{\Lambda^{\prime}}$, so (2.1) defines a linear functional on $\bigcup_{A \text { finite }} \mathscr{A}_{\Lambda}$. This functional is also obviously translation invariant and normalized to $\omega(\mathbb{1})=1$. But without further assumptions $\omega$ will rarely be positive. For this reason we had to assume positivity from the outset, by applying the proposition only to states. In order to turn formula 2.1 into a useful tool for constructing states we need conditions, which will ensure the positivity of $\omega$.

Necessary and sufficient conditions are given in the next proposition, using the concept of matrix order. A matrix order for a vector space $\mathscr{B}$ is an ordering of each of the spaces $\mathscr{M}_{n} \otimes \mathscr{B}$ of $n \times n$-matrices with entries in $\mathscr{B}$, such that these orderings satisfy a certain consistency condition. Since a finite-dimensional $C^{*}$-algebra $\mathscr{A}$ is a direct sum of matrix algebras, $\mathscr{A} \otimes \mathscr{B}$ is matrix ordered in a canonical way, for any matrix ordered $\mathscr{B}$. A completely positive map $T: \mathscr{B}_{1} \rightarrow \mathscr{B}_{2}$

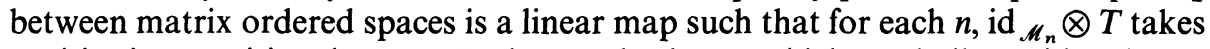
positive into positive elements. In the standard case, which we shall consider almost exclusively, $\mathscr{B}$ is a $C^{*}$-algebra and $\mathscr{M}_{n} \otimes \mathscr{B}$ is equipped with its ordering as a $C^{*}$-algebra. Completely positive maps between operator algebras are well studied [60]. Since many of our results make use of the detailed structure theory of completely positive maps on $C^{*}$-algebras, notably the Stinespring dilation theorem [59], we could not extend our theory to states generated by completely positive maps on a general matrix ordered space. Therefore we collected the basic definitions and results concerning matrix order in Appendix 1, where we also prove the non-trivial direction of Proposition 2.3.

2.3 Proposition. Let $\mathscr{A}$ be a finite-dimensional $C^{*}$-algebra, and $\mathscr{B}$ a finite-dimensional matrix ordered space with $e \in \mathscr{B}$ positive, and $\rho \in \mathscr{B}^{*}$ a positive linear functional. Let $\mathbb{E}: \mathscr{A} \otimes \mathscr{B} \rightarrow \mathscr{B}$ be a completely positive map such that

$$
\mathbb{E}\left(\mathbb{1}_{\mathscr{A}} \otimes e\right)=e, \quad \text { and } \quad \rho\left(\mathbb{E}\left(\mathbb{1}_{\mathscr{A}} \otimes B\right)\right)=\rho(B), \quad B \in \mathscr{B} .
$$

Then with $\mathbb{E}_{A}(B)=\mathbb{E}(A \otimes B)$, these objects generate a finitely correlated state $\omega$, and every finitely correlated state is of this form.

It is easy to see that complete positivity of $\mathbb{E}$ ensures positivity of $\omega$, by introducing the "iterates" $\mathbb{E}^{(n)}: \mathscr{A}{ }^{\otimes n} \otimes \mathscr{B} \rightarrow \mathscr{B}$ with $\mathbb{E}^{(1)}=\mathbb{E}$, and

$$
\mathbb{E}^{(n+1)}=\mathbb{E}\left(\mathrm{id}_{\mathscr{A}} \otimes \mathbb{E}^{(n)}\right): \mathscr{A} \otimes \mathscr{A}^{\otimes n} \otimes \mathscr{B} \rightarrow \mathscr{A} \otimes \mathscr{B} \rightarrow \mathscr{B} .
$$

Then $\mathbb{E}^{(n)}$ is completely positive, since this property is conserved under composition and tensoring with identity maps. Hence by (2.1) $A_{1} \otimes \cdots A_{n} \mapsto \omega\left(A_{1} \otimes \cdots A_{n}\right)=$ $\rho(e)^{-1} \rho\left(\mathbb{E}^{(n)}\left(A_{1} \otimes \cdots A_{n} \otimes \mathbb{1}_{\mathscr{B}}\right)\right)$ is positive.

2.4 Definition. Let $\mathscr{A}$ be a (not necessarily finite-dimensional) $C^{*}$-algebra with unit. Then if the positivity conditions of Proposition 2.3 are satisfied, and $\mathscr{B}$ is a finite-dimensional $C^{*}$-algebra with its canonical matrix order, $\omega$ will be called the 
$C^{*}$-finitely correlated state generated by $(\mathbb{E}, \rho, e)$. The set of $C^{*}$-finitely correlated states on $\mathscr{A}_{\mathbb{Z}}$ will be denoted by $\mathscr{F}$, or $\mathscr{F}(\mathscr{A})$.

Example 1. We now introduce a one-parameter family of $C^{*}$-finitely correlated states which will turn out to contain the ground state of the spin 1 antiferromagnet introduced in [5], (we will call the latter model the AKLT model). As we are working with a spin 1 chain the single site observable algebra $\mathscr{A}$ consists of the $3 \times 3$ complex matrices $\mathscr{M}_{3}$. For the auxiliary algebra $\mathscr{B}$ we take the smallest non-trivial matrix algebra $\mathscr{B}=\mathscr{M}_{2}$. We will label our states with a parameter $\theta \in[0, \pi)$. In order to describe the three defining objects $\left(\mathbb{E}_{\theta}, \rho_{\theta}, e_{\theta}\right)$ we first introduce a linear map $V_{\theta}: \mathbb{C}^{2} \rightarrow \mathbb{C}^{3} \otimes \mathbb{C}^{2}$. Let $\left| \pm \frac{1}{2}\right\rangle$ and $|-1\rangle,|0\rangle,|1\rangle$ denote orthonormal bases of $\mathbb{C}^{2}$ and $\mathbb{C}^{3}$ respectively. Later on we will identify these basis vectors with the eigenvectors of the $z$-component of spin. $V_{\theta}$ is now explicitly given as:

$$
V_{\theta}\left|\frac{1}{2}\right\rangle=\cos \theta\left|1,-\frac{1}{2}\right\rangle-\sin \theta\left|0, \frac{1}{2}\right\rangle, \quad V_{\theta}\left|-\frac{1}{2}\right\rangle=\sin \theta\left|0,-\frac{1}{2}\right\rangle-\cos \theta\left|-1, \frac{1}{2}\right\rangle .
$$

Considering $V_{\theta}$ as a $6 \times 2$ matrix we now define:

(1) $\mathbb{E}_{\theta}: \mathscr{M}_{3} \otimes \mathscr{M}_{2} \rightarrow \mathscr{M}_{2}: A \otimes B \mapsto V_{\theta}^{*} A \otimes B V_{\theta}$,

(2) $\rho_{\theta}: \mathscr{M}_{2} \rightarrow \mathbb{C}: B \mapsto \frac{1}{2} \operatorname{Tr} B$,

(3) $e_{\theta}=\mathbb{1} \in \mathscr{M}_{2}$.

It is well-known that a map of the form $X \mapsto \sum_{i} V_{i}^{*} X V_{i}$ is completely positive [62].

It remains to be checked that the relations (2.2) are satisfied. As $V_{\theta}$ is an isometry, $\mathbb{E}_{\theta}\left(\mathbb{1}_{\mathscr{M}_{3}} \otimes \mathbb{1}_{\mathscr{M}_{2}}\right)=V_{\theta}^{*} V_{\theta}=\mathbb{1}_{\mathscr{M}_{2}}$ and $\rho_{\theta}\left(\mathbb{E}_{\theta}(\mathbb{1} \otimes B)\right)=\rho_{\theta}(B)$ follows from $\operatorname{Tr}_{\mathbb{C}^{3}}$ $V_{\theta} V_{\theta}^{*}=\mathbb{1}_{\mathscr{M}_{2}} . \omega_{\theta}$ is then the state on $\left(\mathscr{M}_{3}\right)_{\mathbb{Z}}$ constructed as in formula (2.1). We will see later on that for $\cos \theta=\sqrt{2 / 3}, \omega_{\theta}$ coincides with the ground state of the AKLT model.

For $C^{*}$-algebras the above argument that complete positivity of $\mathbb{E}$ implies positivity of $\omega$ is independent of $\mathscr{A}$ or $\mathscr{B}$ being finite-dimensional. If we drop the restrictions on $\mathscr{B},(2.1)$ yields every translation invariant state $\omega$ on $\mathscr{A}_{\mathbb{Z}}$. To see this it suffices to take $\mathscr{B}:=\mathscr{A}_{\mathbb{N}}$, and $\mathbb{E}\left(A \otimes\left(A_{1} \otimes \cdots A_{n}\right)\right)=A \otimes A_{1} \otimes \cdots A_{n}$, and to extend this map by linearity and continuity to all of $\mathscr{A} \otimes \mathscr{B}$. The state $\rho$ is then taken as the restriction of the given translation invariant state $\omega$ on $\mathscr{A}_{\mathbb{Z}}$ to the subalgebra $\mathscr{A}_{\mathbb{N}}$. It is evident that with these definitions the original state $\omega$ satisfies (2.1). Hence it is mainly the finite dimension of $\mathscr{B}$, which gives a non-trivial content to Definition 2.4 .

There is also a version of our construction for $W^{*}$-algebras $\mathscr{A}$ : the tensor product in the definition of the $n$-step algebra $\mathscr{A}_{\{i+1, \ldots l+n\}}$ is then taken as the $W^{*}$-tensor product, and the algebra $\mathscr{A}_{\mathbb{Z}}$ is the $C^{*}$-inductive limit of these algebras. Since the category of normal completely positive maps between $W^{*}$-algebras is closed under composition and tensor products, the above argument also shows that provided $\mathbb{E}$ is normal and completely positive (and if $\mathscr{B}$ is also allowed to be an infinite-dimensional $W^{*}$-algebra, provided also $\rho$ is a normal state), then formula 2.1 defines a locally normal state on $\mathscr{A}_{\mathbb{Z}}$.

It is useful to note that the objects generating a $C^{*}$-finitely correlated state $\omega$ can be chosen in the standard form described in the following lemma. We shall use this form whenever convenient. 
2.5 Lemma. Any $C^{*}$-finitely correlated state $\omega$ is also generated by some $\mathbb{E}, \rho, e$ such that $e=\mathbb{1}$ is the identity of the algebra $\mathscr{B}$, and $\rho$ is a faithful state on $\mathscr{B}$. Moreover, $\mathscr{B}$ may be taken either to be minimal in the sense that no proper subalgebra contains $\mathbb{1}$ and is invariant under all $\mathbb{E}_{A}$, or may be taken as a full matrix algebra $\mathscr{B}=\mathscr{M}_{k}$.

Proof. If $0 \leqq B \leqq \lambda e$ for some $B \in \mathscr{B}$, and $0 \leqq A \in \mathscr{A}$, then $0 \leqq \mathbb{E}(A \otimes B) \leqq$ $\|A\| \mathbb{E}(\mathbb{1} \otimes B) \leqq \lambda\|A\| \mathbb{E}(\mathbb{1} \otimes e)=\lambda\|A\| e$. Hence the subalgebra $\tilde{\mathscr{B}}=e \mathscr{B} e$ generated by elements dominated by $e$ is a common invariant subspace of all operators $\mathbb{E}_{A}$. Hence the restriction of $\mathbb{E}$ to $\mathscr{A} \otimes \widetilde{\mathscr{B}}$ also generates $\omega$, and we may suppose that $e$ is invertible in the algebra $\tilde{\mathscr{B}}$ generating $\omega$. Clearly, $\omega$ is also generated from $\tilde{\mathbb{E}}, \tilde{\rho}, \mathbb{1}_{\tilde{B}}$ with $\tilde{\mathbb{E}}(A \otimes B)=e^{-1 / 2} \mathbb{E}\left(A \otimes e^{1 / 2} B e^{1 / 2}\right) e^{-1 / 2}$, and $\tilde{\rho}(B)=\rho\left(e^{1 / 2} B e^{1 / 2}\right)$. Hence we may take $e=\mathbb{1}$.

Suppose that $\rho$ is not strictly positive, i.e. $s:=\operatorname{supp}(\rho)<\mathbb{1}$. Consider the operator $P: B \mapsto s B s$ on $\mathscr{B}$. Then since the functionals $\rho^{\prime}=\rho \circ \mathbb{E}_{A_{1}} \circ \cdots \mathbb{E}_{A_{n}} \in \mathscr{B}^{*}$ are all dominated by $\rho$, we have $\rho^{\prime} \circ P=\rho^{\prime}$. Hence $\omega$ is also represented by $\widetilde{B}=s B s \subset \mathscr{B}$ with $\mathbb{1}_{\mathscr{B}}=P \mathbb{1}_{\mathscr{B}}=s, \tilde{\rho}=\rho\left(\mathbb{1}_{\mathscr{B}}\right)^{-1} \cdot \rho \mid \tilde{\mathscr{B}}$, and $\tilde{\mathbb{E}}_{A}=P \circ \mathbb{E}_{a} \mid \tilde{\mathscr{B}}$. The statement about minimality is obvious. Since $\mathscr{B}=\bigoplus_{\alpha} \mathscr{M}_{k_{\alpha}}$ is a finite direct sum of matrix algebras, we may pick a representation on $\stackrel{\alpha}{ }^{k}=\bigoplus \mathbb{C}^{k_{\alpha}}$. Let $P_{\alpha}$ be the projection onto the $\alpha^{\text {th }}$ summand and $\mathbb{P}: \mathscr{M}_{k} \rightarrow \mathscr{B}: B \mapsto \sum_{\alpha} P_{\alpha} B P_{\alpha}$. Then $\tilde{\mathbb{E}}:=\mathbb{P} \circ \mathbb{E} \circ($ id $\mathscr{A} \otimes \mathbb{P}): \mathscr{A} \otimes \mathscr{M}_{k} \rightarrow$ $\mathscr{M}_{k}$ generates the same state.

Example 2. We now compute the minimal representation of the states $\omega_{\theta}$ defined in Example 1.

(1) The case $\cos \theta \sin \theta \neq 0$. One can check that already $\mathbb{E}_{\theta}\left(\mathscr{M}_{3} \otimes \mathbb{1}_{\mathscr{M}_{2}}\right)=\mathscr{M}_{2}$ and therefore $\mathscr{M}_{2}$ is a minimal $\mathscr{B}$ for the state $\omega_{\theta}$. And furthermore, as $\rho_{\theta}(B)=\frac{1}{2} \operatorname{Tr} B$, $\rho_{\theta}$ is a faithful state on $\mathscr{M}_{2}$.

(2) The case $\theta=0$. We have

$$
\begin{aligned}
\mathbb{E}_{0}(A \otimes B)\left|\frac{1}{2}\right\rangle & =\langle 1|A| 1\rangle\left\langle-\frac{1}{2}|B|-\frac{1}{2}\right\rangle\left|\frac{1}{2}\right\rangle-\langle-1|A| 1\rangle\left\langle\frac{1}{2}|B|-\frac{1}{2}\right\rangle\left|-\frac{1}{2}\right\rangle, \\
\mathbb{E}_{0}(A \otimes B)\left|-\frac{1}{2}\right\rangle & =-\langle 1|A|-1\rangle\left\langle-\frac{1}{2}|B| \frac{1}{2}\right\rangle\left|\frac{1}{2}\right\rangle+\langle-1|A|-1\rangle\left\langle\frac{1}{2}|B| \frac{1}{2}\right\rangle\left|-\frac{1}{2}\right\rangle .
\end{aligned}
$$

Hence $\mathbb{E}_{0}\left(\mathscr{M}_{3} \otimes \mathbb{1}_{\mathscr{M}_{2}}\right)$ consists now of the diagonal matrices of $\mathscr{M}_{2}$ and it easy to see that this is already the minimal algebra. Again $\rho_{0}=\frac{1}{2} \operatorname{Tr}$ is faithful on this algebra.

(3) The case $\theta=\pi / 2$. $\mathbb{E}_{\pi / 2}(A \otimes B)=\langle 0|A| 0\rangle \sigma^{z} B \sigma^{z}$, where $\sigma^{z}$ is the usual Pauli matrix. Obviously the minimal algebra is $\mathbb{C}$. Therefore $\mathbb{E}_{\pi / 2}$ restricted to the minimal algebra is now the state $A \in \mathscr{M}_{3} \mapsto\langle 0|A| 0\rangle$. It follows that $\omega_{\pi / 2}$ is a product state on the chain.

The following proposition lists some basic properties of the class of $C^{*}$-finitely correlated states. For (3) and (4) of Proposition 2.6 we use the identification of $\left(\mathscr{A}^{\otimes p}\right)_{\mathbb{Z}}$ and $\mathscr{A}_{\mathbb{Z}}$ mentioned in the beginning of this section. 


\subsection{Proposition}

(1) Symmetric product states are in $\mathscr{F}$.

(2) $\mathscr{F}$ is convex.

(3) For $p \in \mathbb{N}, \omega \in \mathscr{F}$ is also $C^{*}$-finitely correlated as a state on $\left(\mathscr{A}^{\otimes p}\right)_{\mathbb{Z}}$.

(4) Conversely, let $\omega$ be a p-periodic state on $\mathscr{A}_{\mathbb{Z}}$, which is $C^{*}$-finitely correlated as a state on $\left(\mathscr{A}^{\otimes p}\right)_{\mathbb{Z}}$. Let $\bar{\omega}=\frac{1}{p} \sum_{r=0}^{p-1} \omega^{\circ} \alpha_{r}$ be the average of $p$ consecutive translates of $\omega$. Then $\bar{\omega} \in \mathscr{F}$.

(5) $\mathscr{F}$ is *weakly dense in the set $\mathscr{T}$ of translation invariant states on $\mathscr{A}_{\mathbb{Z}}$.

Proof. (1) Let $\omega\left(A_{n} \otimes \cdots A_{n+m}\right)=\prod_{i} \eta\left(A_{i}\right)$. Then $\omega$ is generated by $\mathscr{B}=\mathbb{C}, \rho(\lambda)=\lambda$, $e=1$, and $\mathbb{E}(A \otimes \lambda)=\lambda \eta(A)$.

(2) Let $\omega=\sum_{i} \lambda_{i} \omega_{i}$ with $\lambda_{i}>0$ and $\omega_{i}$ generated by $\left(\mathscr{B}_{i}, \rho_{i}, \mathbb{E}_{i}, e_{i}\right)$. Set $\mathscr{B}=\bigoplus_{i} \mathscr{B}_{i}$, $\rho=\bigoplus_{i} \lambda_{i} \rho_{i}, e=\bigoplus_{i} e_{i}$, and $\mathbb{E}=\bigoplus_{i} \mathbb{E}_{i}$. Since $\mathbb{E}$ maps each direct summand of $\mathscr{B}$ into itself, we also have $\mathbb{E}_{A_{1}} \circ \ldots \circ \mathbb{E}_{A_{n}}=\bigoplus_{i} \mathbb{E}_{i, A_{1}} \circ \ldots \circ \mathbb{E}_{i, A_{n}}$. Evaluating this at $e$ and applying $\rho$ we conclude that $\omega$ is generated by $(\mathbb{E}, \rho, e)$.

(3) If $\omega$ as a state on $\mathscr{A}_{\mathbb{Z}}$ is generated by $(\mathbb{E}, \rho, e)$, then as a state on $\left(\mathscr{A}^{\otimes p}\right)_{\mathbb{Z}}$ it is generated by $\left(\mathbb{E}^{(p)}, \rho, e\right)$, where $\mathbb{E}^{(p)}$ is the $p^{\text {th }}$ iterate of $\mathbb{E}$.

(4) Suppose now that the $p$-periodic state $\omega$ is generated by $\rho \in \mathscr{B}^{*}, e=\mathbb{1} \in \mathscr{B}$, and $\mathbb{E}: \mathscr{A}^{\otimes p} \otimes \mathscr{B} \rightarrow \mathscr{B}$. We set $\tilde{\mathscr{B}}=\bigoplus_{r=0} \mathscr{A}^{\otimes r} \otimes \mathscr{B}$, with the convention $\mathscr{A}^{\otimes 0} \otimes \mathscr{B}=\mathscr{B}$. We denote the $r^{\text {th }}$ component of $\tilde{B} \in \tilde{\mathscr{B}}$ by $\tilde{B}_{r}$. For $A \in \mathscr{A}$ let

$$
\tilde{\mathbb{E}}(A \otimes \widetilde{B})_{r}= \begin{cases}\mathbb{E}\left(A \otimes \widetilde{B}_{p-1}\right), & \text { if } r=0 \\ A \otimes \widetilde{B}_{r-1}, & \text { if } 1 \leqq r \leqq p-1 .\end{cases}
$$

The state $\tilde{\rho} \in \tilde{\mathscr{B}}^{*}$ is defined by

$$
\tilde{\rho}(\tilde{B})=\frac{1}{p} \sum_{r=0}^{p-1} \rho\left(\mathbb{E}\left(\mathbb{1}_{\mathscr{A}}^{\otimes(p-r)} \otimes \tilde{B}_{r}\right)\right) .
$$

Note that by the invariance property of $\mathbb{E}$ the summand with $r=0$ is just $\rho\left(\widetilde{B}_{0}\right)$. One checks that indeed $\tilde{\rho} \circ \widetilde{\mathbb{E}}_{\mathbb{1}}=\tilde{\rho}$, and $\tilde{\mathbb{E}}(\mathbb{1})=\mathbb{1}$, so $\tilde{\rho}$ and $\tilde{\mathbb{E}}$ define a translation invariant state on $\mathscr{A}_{\mathbb{Z}}$. It is clear that the $p^{\text {th }}$ iterate of $\tilde{\mathbb{E}}$ maps each of the summands of $\tilde{\mathscr{B}}$ into itself. In fact:

$$
\left(\tilde{\mathbb{E}}_{A_{1}} \circ \cdots \circ \tilde{\mathbb{E}}_{A_{p}}(\tilde{B})\right)_{r}=A_{1} \otimes \cdots A_{r} \otimes \mathbb{E}\left(A_{r+1} \otimes \cdots A_{p} \otimes \tilde{B}_{r}\right),
$$

and

$$
\begin{aligned}
\left(\tilde{\mathbb{E}}_{A_{1}} \circ \cdots \circ \tilde{\mathbb{E}}_{A_{n p}}\left(\mathbb{1}_{\mathscr{B}}\right)\right)_{r}= & A_{1} \otimes \cdots A_{r} \otimes \mathbb{E}_{\left(A_{r+1} \otimes \cdots A_{p+r+1}\right)} \\
& \circ \cdots \circ \mathbb{E}\left(A_{(n-1) p+r+1} \otimes \cdots A_{n p} \otimes \mathbb{1}_{\mathscr{A}}^{\otimes r} \otimes \mathbb{1}_{\mathscr{B}}\right) .
\end{aligned}
$$

Evaluating this on the state $\tilde{B}_{r} \mapsto \rho\left(\mathbb{E}\left(\mathbb{1}_{\mathscr{A}}^{\otimes(p-r)} \otimes \tilde{B}_{r}\right)\right)$ gives the $\omega$-expectation of $\mathbb{1}_{\mathscr{A}}^{\otimes(p-r)} \otimes A_{1} \otimes \cdots A_{n p} \otimes \mathbb{1}_{\mathscr{A}}^{\otimes r}$, i.e. the expectation of $A_{1} \otimes \cdots A_{n p}$ in $\omega \circ \alpha_{r}$. The result follows by summing over $r$. 
(5) Let $\omega$ be a translation invariant state. Consider the product state $\omega^{\prime}$ on $\left(\mathscr{A}^{\otimes p}\right)_{\mathbb{Z}}$ formed from the $p$-site restriction of $\omega$. Let $\omega^{p}=\bar{\omega}^{\prime}$. Then by (1) and (4) $\omega^{p} \in \mathscr{F}$. The states $\omega$ and $\omega^{\prime} \circ \alpha_{r}$ coincide on observables $A=A_{i+1} \otimes \cdots A_{i+n}$ for $n<p$, unless the interval $i+1, \ldots i+n$ contains one of the "breakpoints" $n p+r$. Thus $\left|\omega(A)-\omega^{p}(A)\right| \leqq\left(1-\frac{2(n-1)}{p}\right)\|A\|$, and $w^{*}-\lim _{p} \omega^{p}=\omega$.

We close this section with an alternative construction for $C^{*}$-finitely correlated states. It is a generalization of the "valence bond solid" states of [17-21]. For constructing a state on the chain $\mathscr{A}_{\mathbb{Z}}$ according to this scheme, we need two auxiliary finite-dimensional $C^{*}$-algebras $\mathscr{B}$ and $\mathscr{B}$. The state is determined by a completely positive map $\mathbb{F}: \mathscr{A} \rightarrow \mathscr{B} \otimes \overline{\mathscr{B}}$, and a state $\Phi: \overline{\mathscr{B}} \otimes \mathscr{B} \rightarrow \mathbb{C}$, which have to satisfy the compatibility conditions

$$
\left(\mathrm{id}_{\mathscr{B}} \otimes \Phi\right)\left(\mathbb{F}\left(\mathbb{1}_{\mathscr{A}}\right) \otimes \mathbb{1}_{\mathscr{B}}\right)=\mathbb{1}_{\mathscr{B}} \quad \text { and } \quad\left(\Phi \otimes \mathrm{id}_{\overline{\mathscr{B}}}\right)\left(\mathbb{1}_{\overline{\mathscr{B}}} \otimes \mathbb{F}\left(\mathbb{1}_{\mathscr{A}}\right)\right)=\mathbb{1}_{\overline{\mathscr{B}}} .
$$

On any $n$ consecutive sites a state $\omega$ is then defined by

$$
\omega\left(A_{1} \otimes \cdots A_{n}\right)=\underbrace{\Phi \otimes \cdots \Phi}_{n+1}\left(\mathbb{1}_{\overline{\mathscr{B}}} \otimes \mathbb{F}\left(A_{1}\right) \otimes \cdots \mathbb{F}\left(A_{n}\right) \otimes \mathbb{1}_{\mathscr{B}}\right) .
$$

Again, the compatibility conditions ensure that the hierarchy of functionals thus defined for different $n$ determines a translation invariant state on $\mathscr{A}_{\mathbf{Z}}$. Any state, which can be obtained in this way will be called a valence bond state. The construction can be visualized as follows:

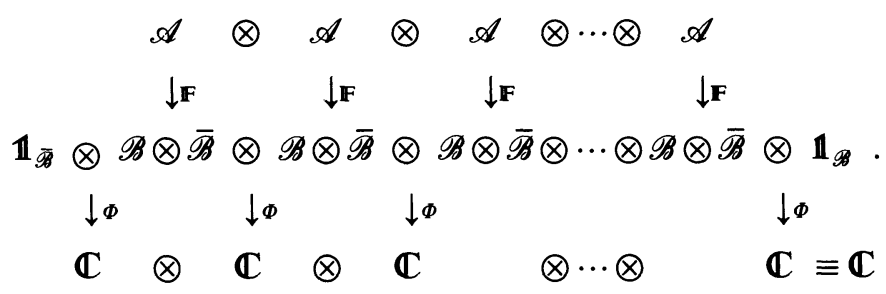

Fig. 1. The VBS construction

The connection to the class of $C^{*}$-finitely correlated states is made in the following proposition.

2.7 Proposition. Every $C^{*}$-finitely correlated state is a valence bond state and conversely. Moreover, in the representation of a valence bond state we may take $\mathscr{B} \cong \overline{\mathscr{B}} \cong \mathscr{M}_{k}$, and $\Phi$ to be a pure state with faithful restriction to either factor.

Proof. Given a valence bond state, we define

$$
\mathbb{E}(A \otimes B)=\left(\mathrm{id}_{\mathscr{B}} \otimes \Phi\right)(\mathbb{F}(A) \otimes B) \quad \text { and } \quad \rho(B)=\Phi\left(\mathbb{1}_{\overline{\mathscr{B}}} \otimes B\right) .
$$

Then the compatibility conditions for $\mathbb{F}$ and $\Phi$ become those for $\mathbb{E}$ and $\rho$, and one checks by induction on $n$, that $\omega$ is generated by $\mathbb{E}, \rho$. The converse and remaining statements will be shown using dilation theory in Sect. 4. 
Example 3. We will now give the valence bond description of $\omega_{\theta}$, i.e. we will specify the maps $\mathbb{F}_{\theta}$ and $\Phi_{\theta}$ appearing in Fig. 1 . We take $\mathscr{B}=\overline{\mathscr{B}}=\mathscr{M}_{2}$. In order to define $\mathbb{F}_{\theta}$ we introduce a linear map $W_{\theta}: \mathbb{C}^{3} \rightarrow \mathbb{C}^{2} \otimes \mathbb{C}^{2}$. With the same notation for the basis vectors:

$$
\begin{aligned}
W_{\theta}|1\rangle & =\sqrt{2} \cos \theta\left|\frac{1}{2}, \frac{1}{2}\right\rangle, \\
W_{\theta}|0\rangle & =\sin \theta\left(\left|\frac{1}{2},-\frac{1}{2}\right\rangle+\left|-\frac{1}{2}, \frac{1}{2}\right\rangle\right), \\
W_{\theta}|-1\rangle & =\sqrt{2} \cos \theta\left|-\frac{1}{2},-\frac{1}{2}\right\rangle .
\end{aligned}
$$

The maps $\mathbb{F}_{\theta}$ and $\Phi_{\theta}$ are given by:

$$
\mathbb{F}_{\theta}(A)=W_{\theta} A W_{\theta}^{*} \quad \Phi_{\theta}\left(B_{1} \otimes B_{2}\right)=\left\langle\varphi \mid B_{1} \otimes B_{2} \varphi\right\rangle,
$$

where $\varphi$ is the singlet vector $\sqrt{1 / 2}\left|\frac{1}{2},-\frac{1}{2}\right\rangle-\sqrt{1 / 2}\left|-\frac{1}{2}, \frac{1}{2}\right\rangle$. We can now verify the compatibility conditions (2.3) and thus obtain a valence bond state. We claim that this state coincides with $\omega_{\theta}$. For this to be true the formulae (2.4) should reproduce the $\mathbb{E}_{\theta}$ and $\rho_{\theta}$ that were used to define $\omega_{\theta}$ as a $C^{*}$-finitely correlated state. And indeed, this follows from

$$
V_{\theta}=\left(W_{\theta}^{*} \otimes \mathbb{1}_{\mathscr{M}_{2}}\right)\left(\mathbb{1}_{\mathscr{M}_{2}} \otimes \varphi\right), \quad \frac{1}{2} \operatorname{Tr} B=\Phi_{\theta}\left(\mathbb{1}_{\mathscr{M}_{2}} \otimes B\right), \quad B \in \mathscr{M}_{2}
$$

The reader can check that we have reproduced here the construction that appears in the proof of Proposition 2.7 at the end of Sect. 4. The anti-unitary operator $\chi \mapsto \bar{\chi}$ in that proof has to be taken in our case: $\left|\frac{1}{2}\right\rangle \mapsto-\left|-\frac{1}{2}\right\rangle,\left|-\frac{1}{2}\right\rangle \mapsto\left|\frac{1}{2}\right\rangle$. In the case of the AKLT model (where $\cos \theta=\sqrt{2 / 3}$ ), $\mathbb{F}$ equals $\frac{4}{3} \times$ the embedding of $\mathbb{C}^{3}$ as state space of a spin 1 into the state space $\mathbb{C}^{2} \otimes \mathbb{C}^{2}$ of two spin $1 / 2$ 's.

In spite of the equivalence obtained in Proposition 2.7 both the $C^{*}$-finitely correlated and valence bond representation have their own merits. For discussing states on the chain we found the formalism involving the map $\mathbb{E}$ far more useful. For example, the computation of correlation functions and their cluster properties, for which the valence bond picture suggests a rather involved diagrammatic technique [5], is reduced to determining the spectrum of a finite matrix. On the other hand, the main virtue of the valence bond structure is that it generalizes immediately to graphs other than the one-dimensional chain: an algebra $\mathscr{A}_{i}$ is then associated to any vertex " $i$ " of the graph, and an algebra $\mathscr{B}_{i j}$ to each directed edge. The two basic kinds of completely positive maps are then a map $\mathbb{F}_{i}$ taking each $\mathscr{A}_{i}$ into the tensor product of the outgoing edge algebras, and "contractions" $\mathscr{B}_{i j} \otimes \mathscr{B}_{j i} \rightarrow \mathbb{C}$. With each set $\Lambda$ of vertices one associates the "observable algebra" $\mathscr{A}_{\Lambda}=\bigotimes_{i \in \Lambda} \mathscr{A}_{i}$ and the "outgoing edge algebra" $\mathscr{B}_{\partial \Lambda}=\bigotimes_{i \in \Lambda, j \neq \Lambda} \mathscr{B}_{i j}$. Clearly, every state on $\mathscr{B}_{\partial \Lambda}$ is transformed via the $\mathbb{F}_{i}$ and the contractions into a state on $\mathscr{A}_{\Lambda}$. In order to get an explicit definition of a state on the infinite system out of this scheme one either has to show the existence of a unique limit state, independent of the choice of states for $\mathscr{B}_{\partial \Lambda}$ (which serve as "boundary conditions"), or one has to find and verify appropriate compatibility conditions for these states. Both problems appear to be highly nontrivial. Some results about a two-dimensional example have been obtained in [46]. Some general answers can be given in the case of the Bethe lattice (Cayley tree) [32]. 


\section{Ergodic Decompositions}

It is known that the extreme points of the set $\mathscr{T}$ of translation invariant states, called "ergodic" states, are characterized by the decay of their correlation functions. For a $C^{*}$-finitely correlated state the correlation functions can be given explicitly, and we shall now utilize this to obtain the ergodic decomposition of any $C^{*}$-finitely correlated state. The behavior of correlation functions of any finitely correlated state $\omega$ is determined by the map $\hat{\mathbb{E}}:=\mathbb{E}_{\mathbb{1}}: \mathscr{B} \rightarrow \mathscr{B}$ through the equation

$$
\omega(A_{n} \otimes \underbrace{\mathbb{1} \cdots \otimes \mathbb{1}}_{m-1} \otimes A_{n+m})=\left(\rho \circ \mathbb{E}_{A_{n}}\right) \circ(\hat{\mathbb{E}})^{m-1}\left(\mathbb{E}_{A_{n+m}}\left(\mathbb{1}_{\mathscr{B}}\right)\right) .
$$

Thus determining the $m$-dependence of all these functions reduces to a standard task from linear algebra, namely computing all powers of the matrix $\hat{\mathbb{E}}$, e.g. by diagonalization.

3.1 Proposition. Let $\omega$ be a $C^{*}$-finitely correlated state on $\mathscr{A}_{\mathbb{Z}}$. Then the following are equivalent:

(1) $\omega$ is extremal in the convex set $\mathscr{F}$ of $C^{*}$-finitely correlated states.

(2) $\omega$ is ergodic, i.e. extremal in the convex set $\mathscr{T}$ of translation invariant states.

(3) $\omega$ is the $C^{*}$-finitely correlated state generated by some $(\mathbb{E}, \rho, e)$ such that $e=\mathbb{1}$ is the only eigenvector of $\hat{\mathbb{E}}$ with eigenvalue one.

Proof. (2) $\Rightarrow(1)$ is trivial.

$(3) \Rightarrow(2)$ : Consider the Jordan decomposition of $\hat{\mathbb{E}}$, i.e. $\hat{\mathbb{E}}=\sum_{\lambda}\left(\lambda P_{\lambda}+R_{\lambda}\right)$, where the sum runs over all eigenvalues, $P_{\lambda} P_{\lambda^{\prime}}=\delta_{\lambda \lambda^{\prime}} P_{\lambda}$, and $R_{\lambda}^{\lambda}$ is nilpotent with $P_{\lambda} R_{\lambda^{\prime}}=R_{\lambda^{\prime}} P_{\lambda}=\delta_{\lambda \lambda^{\prime}} R_{\lambda}$. Since $\|\hat{\mathbb{E}}\| \leqq 1$ we have $R_{\lambda}=0$ for $\lambda$ with $|\lambda|=1$. (Otherwise there would be a vector $B \in \mathscr{B}$ such that $R_{\lambda} B \neq 0$ and $R_{\lambda}^{2} B=0$, making the sequence $\hat{\mathbb{E}}^{n}(B)=\lambda^{n} B+n \lambda^{n-1} R_{\lambda} B$ unbounded.) Therefore we may find for every $\varepsilon>0$ convex combination coefficients $\mu_{n}, n \in \mathbb{N}$, such that $\left\|P_{1}-\sum_{n} \mu_{n} \hat{\mathbb{E}}^{n}\right\| \leqq \varepsilon$. Since by assumption $P_{1}$ is one-dimensional, this implies the clustering condition $[16,4.3 .10,4.3 .11]$ uniformly for all correlation functions. Hence $\omega$ is ergodic.

$(1) \Rightarrow(3)$ : According to Lemma 2.5 we can choose a representation of $\omega$ with $e=\mathbb{1}$. Consider the cone $\Gamma=\{e \in \mathscr{B} \mid e \geqq 0, \hat{\mathbb{E}} e=e\}$. Then for each $e \in \Gamma$ let $\omega_{e}$ be the $C^{*}$-finitely correlated state generated by $\rho, \mathbb{E}, e$. We claim that $e$ is extremal in $\Gamma$, iff there is no $e^{\prime} \in \Gamma$ such that $\operatorname{supp} e^{\prime}<\operatorname{supp} e$. In fact, if $\mu e \geqq e^{\prime} \neq 0$ and $e^{\prime}$ not proportional to $e$, then also $e>e^{\prime \prime}=e-\alpha e^{\prime}$ for all $\alpha \geqq 0$, and by choosing the largest $\alpha$ consistent with $e^{\prime \prime} \geqq 0$, we obtain a non-zero $e^{\prime \prime} \in \bar{\Gamma}$, which is also dominated by $e$, and satisfies $\operatorname{supp} e^{\prime \prime}<\operatorname{supp} e$. Conversely, $\operatorname{supp} e^{\prime}<\operatorname{supp} e$ implies $e^{\prime} \leqq \mu e$ for some $\mu$ and $e^{\prime}$ not proportional to $e$.

If $\omega=\omega_{\mathbb{1}}$ is extremal, all states $\omega_{e}$ are equal as convex components of $\omega$. Hence by taking $e \in \Gamma$ extremal, we may choose a representation of $\omega$ for which the cone $\Gamma$ reduces to the single ray $\mathbb{R}^{+} \mathbb{1}$, i.e. $\mathbb{1}$ is the unique eigenvector with eigenvalue one. Since the adjoint of $\hat{\mathbb{E}}$ has the same spectrum, this also implies that $\rho$ is the unique left eigenvector of $\hat{\mathbb{E}}$.

If 1 is a simple eigenvalue of $\hat{\mathbb{E}}$, then the same is true for the adjoint of $\hat{\mathbb{E}}$. Hence in (3) we could have demanded alternatively that up to a scalar $\rho$ is the 
only element of $\mathscr{B}^{*}$ with $\rho \circ \hat{\mathbb{E}}=\rho$. Therefore, in the ergodic case $\rho$ is determined by $\hat{\mathbb{E}}$, i.e. we need fewer independent data to characterize $\omega$.

3.2 Corollary. $\mathscr{F}$ is a face in $\mathscr{T}$, i.e. in any convex combination $\omega=\sum_{i} \lambda_{i} \omega_{i}$ with $\lambda_{i}>0, \omega_{i} \in \mathscr{T}$, and $\omega \in \mathscr{F}$, we must have $\omega_{i} \in \mathscr{F}$ for all $i$. Moreover, all $\omega_{i}$ can be generated from the same $\mathbb{E}$ with different $\rho$, e. Every $C^{*}$-finitely correlated state has a unique decomposition of this kind, such that each $\omega_{i}$ is also ergodic.

Proof. It is clear from the proof of Proposition 3.1 that we may decompose $\omega$ into states $\omega_{e}$ generated by the same $\mathscr{B}, \mathbb{E}$, which are extremal in $\mathscr{F}$, and hence also extremal in $\mathscr{T}$. Since $\mathscr{T}$ is a simplex $[16,4,3,11]$, such a decomposition of $\omega$ is unique. Thus the $\omega_{e}$ span the face in $\mathscr{T}$ generated by $\omega$.

The condition that the eigenvalue 1 of $\mathbb{E}$ is non-degenerate, does not exclude oscillatory behavior of the correlation functions, which would result from further eigenvalues of modulus 1 . In the Perron-Frobenius theory of Classical Markov chains the set of such eigenvalues, called the "peripheral spectrum" of $\hat{\mathbb{E}}$, is shown to be a group under multiplication. For finite-dimensional $\mathscr{B}$, this implies that all such eigenvalues are roots of unity, so that almost periodic behavior of correlation functions is excluded. The proposition below carries this result over to the quantum case. Examples of $C^{*}$-finitely correlated states, for which $\hat{\mathbb{E}}$ has roots of unity as eigenvalues, are provided by the construction in the proof of Proposition 2.6.(4): in that case, the spectra of $\hat{\tilde{\mathbb{E}}}: \tilde{\mathscr{B}} \rightarrow \mathscr{\mathscr { B }}$ and $\hat{\mathbb{E}}: \mathscr{B} \rightarrow \mathscr{B}$ for the completely positive maps $\tilde{\mathbb{E}}$ and $\mathbb{E}$ generating $\bar{\omega}$ and $\omega$, respectively, are related by

$$
\operatorname{spec}(\hat{\mathbb{E}})=\left\{\lambda \in \mathbb{C} \mid \lambda^{p} \in \operatorname{spec}(\hat{\mathbb{E}})\right\} \text {. }
$$

In particular, the $p^{\text {th }}$ roots of unity are in the spectrum of $\hat{\tilde{E}}$. The converse of this construction can be described as the breaking of translational symmetry, or the detection of Néel order in the state $\omega$. We are then given a $C^{*}$-finitely correlated state and ask whether this state can be represented as a convex combination of $p$-periodic states. The following proposition shows how this symmetry breaking can be detected from the $C^{*}$-finitely correlated representation of a state. We shall call a $p$-periodic state $C^{*}$-finitely correlated, if it is $C^{*}$-finitely correlated as a state on $\left(\mathscr{A}^{\otimes p}\right)_{\mathbb{Z}}$.

3.3 Proposition. Let $\omega$ be an ergodic $C^{*}$-finitely correlated state, and choose $\omega$ to be generated by $(\mathbb{E}, \rho, e)$ such that $e=\mathbb{1}_{\mathscr{B}}$ is the only fixed point of $\hat{\mathbb{E}}$, and $\mathscr{B}$ is generated as a $C^{*}$-algebra by $\left\{\mathbb{E}_{A_{1}} \circ \cdots \circ \mathbb{E}_{A_{n}}(\mathbb{1})\right\}$. Then $\rho$ is faithful, and there is $a$ $p \in \mathbb{N}$ such that

$$
\{\lambda \in \operatorname{spec}(\hat{\mathbb{E}})|| \lambda \mid=1\}=\left\{\exp \left(\frac{2 \pi i}{p} n\right) \mid n=0, \ldots, p-1\right\} .
$$

Each of these eigenvalues is simple, and the corresponding eigenvector can be taken to be a unitary in $\mathscr{B}$. Moreover, $\mathscr{B}$ is a direct sum $\mathscr{B}=\bigoplus_{r=1}^{p} \mathscr{B}_{r}$, and $\mathbb{E}\left(\mathscr{A} \otimes \mathscr{B}_{r}\right) \subset \mathscr{B}_{r-1}$ with $\mathscr{B}_{0} \equiv \mathscr{B}_{p}$. $\omega$ has a unique representation as the average of $p$ p-periodic states, which are translates of each other. These components are again $C^{*}$-finitely correlated.

Proof. It is clear from 3.1 (3) that we can choose a representation as described. 
Since 1 is a non-degenerate eigenvalue of $\mathbb{E}$, there is a unique state $\rho$ with $\rho \circ \hat{\mathbb{E}}=\rho$. Let $s$ be the support projection of $\rho$. Then $\{x \in \mathscr{B} \mid x s=0\}$ is an invariant subspace of $\hat{\mathbb{E}}$, since $x s=0 \Rightarrow \rho\left(\hat{\mathbb{E}}\left(x^{*} x\right)\right)=\rho\left(x^{*} x\right)=0 \Rightarrow s \hat{\mathbb{E}}(x)^{*} \hat{\mathbb{E}}(x) s \leqq s \hat{\mathbb{E}}\left(x^{*} x\right) s=0$. Hence $\hat{\mathbb{E}}$ must have an invariant vector $\tilde{e}$ with $\tilde{e} s=0$, which contradicts the uniqueness of $\mathbb{1}$, unless $s=\mathbb{1}$ and $\rho$ is faithful.

Now consider the positive semidefinite sesquilinear map

$$
\beta: \mathscr{B} \times \mathscr{B} \rightarrow \mathscr{B}:(x, y) \mapsto \hat{\mathbb{E}}\left(x^{*} y\right)-\hat{\mathbb{E}}(x)^{*} \hat{\mathbb{E}}(y) .
$$

It is easy to see $[36,23]$ that $\beta(x, x)=0$ implies $\beta(y, x)=0$ for all $y \in \mathscr{B}$. Now let $u \in \mathscr{B}$ be an eigenvector of $\hat{\mathbb{E}}$, with $\hat{\mathbb{E}} u=e^{i \alpha} u$. Then $\rho(\beta(u, u))=\rho\left(\hat{\mathbb{E}}\left(u^{*} u\right)-u^{*} u\right)=0$ by invariance of $\rho$. Since $\rho$ is faithful $\beta(u, u)=0$, and hence

$$
\hat{\mathbb{E}}(x u)=e^{i \alpha} \hat{\mathbb{E}}(x) u \text { for all } x \in \mathscr{B} .
$$

Also, $u^{*} u$ is invariant under $\hat{\mathbb{E}}$, and hence a multiple of the identity, so that we can take $u$ to be unitary. If $\hat{\mathbb{E}} v=e^{i \gamma} v$, then the above equation gives $\hat{\mathbb{E}}(u v)=e^{i(\alpha+\gamma)} u v$. Hence $u v$ is again an eigenvector of $\hat{\mathbb{E}}$. Since $\hat{\mathbb{E}} u^{*}=e^{-i \alpha} u^{*}$ the peripheral spectrum is a (necessarily finite) group under multiplication, i.e. it consists of the $p^{\text {th }}$ roots of unity for some $p \in \mathbb{N}$. It was already argued in the proof of Proposition 3.1. that peripheral eigenvalues have diagonal Jordan blocks. Moreover, if $u_{1}, u_{2}$ are eigenvectors for the same $e^{i \alpha}, u_{1}^{*} u_{2}$ is invariant under $\hat{\mathbb{E}}$, hence it is a multiple of $\mathbb{1}$, and $u_{1}$ and $u_{2}$ are proportional. This proves that each peripheral eigenvalue is simple.

Let $u$ be the eigenvector with eigenvalue $\lambda=\exp \left(\frac{2 \pi i}{p}\right)$. Then since $u^{p}=\mathbb{1}$ the spectral resolution of $u$ is of the form $u=\sum_{r=1}^{p} \lambda^{r} P_{r}$ with $P_{r}^{*} P_{r^{\prime}}=\delta_{r r^{\prime}} P_{r}$ and $\sum_{r} P_{r}=\mathbb{1}$. The relation $\hat{\mathbb{E}}\left(x u^{r}\right)=\lambda^{r} \hat{\mathbb{E}}(x) u^{r}$ then becomes $\hat{\mathbb{E}}\left(x P_{r}\right)=\hat{\mathbb{E}}(x) P_{r-1}$. Now let $\mathscr{B}_{r}=P_{r} \mathscr{B} P_{r}$, and let $0 \leqq B_{r} \in \mathscr{B}_{r}$, and $0 \leqq A \in \mathscr{A}$. Then $\mathbb{E}\left(A \otimes B_{r}\right) \leqq\|A\| \hat{\mathbb{E}}\left(B_{r}\right)=$ $\|A\| \hat{\mathbb{E}}\left(P_{r} B_{r} P_{r}\right)=\|A\| P_{r-1} \mathbb{E}\left(B_{r}\right) P_{r-1} \leqq\|A\| \cdot\left\|B_{r}\right\| P_{r-1}$. Thus $\mathbb{E}\left(A \otimes B_{r}\right) \in \mathscr{B}_{r-1}$, and this result extends by linearity and continuity to all of $\mathscr{A} \otimes \mathscr{B}_{r}$. It follows that the algebra $\widetilde{\mathscr{B}}=\bigoplus_{r} \mathscr{B}_{r}$ is invariant under all operators $\mathbb{E}_{A}$, and contains $\mathbb{1}$, so that by our minimality assumption $\tilde{\mathscr{B}}=\mathscr{B}$. Clearly, the $p^{\text {th }}$ iterate of $\mathbb{E}^{(p)}: \mathscr{A}^{p} \otimes \mathscr{B} \rightarrow \mathscr{B}$ takes each of the subalgebras $\mathscr{B}_{r}$ into itself. The restriction of $\mathbb{E}^{p}$ to $\mathscr{B}^{r}$ therefore defines a finitely correlated state on $\left(\mathscr{A}^{\otimes p}\right)_{\mathbb{Z}}$. It is easy to check that the resulting $p$ states are translates of each other, and that their average is $\omega$. The uniqueness of this decomposition follows from the uniqueness of ergodic decompositions, applied to the chain $\left(\mathscr{A}^{\otimes p}\right)_{\mathbb{Z}}$.

Combining Corollary 3.2 and Proposition 3.3 we can summarize the results of this section as follows:

3.4 Corollary. Every $C^{*}$-finitely correlated state has a unique decomposition as a finite convex combination of extremal periodic states. These periodic components are again $C^{*}$-finitely correlated.

It should be noted that unlike $\mathscr{T}$, or the set of $p$-periodic states with fixed $p$, the set of all periodic states is not *weakly compact (it is dense in the whole state space), so it is not a priori clear that it has an abundance of extreme points. It is Proposition 3.3, which provides a criterion for the impossibility of decomposing a state into other states of larger period. Together with Proposition 2.6(5) we have 
therefore shown that the *weakly closed convex hull of the extremal periodic states is dense in $\mathscr{T}$. We shall later study a set of $C^{*}$-finitely correlated states, which are even pure as states on $\mathscr{A}_{\mathbb{Z}}$.

Example 4. We close this section by examining the ergodic properties of the states $\omega_{\theta}$. Again we discern three cases, and we immediately use the minimal representations obtained at the end of Sect. 2 in Example 2.

(1) $\cos \theta \sin \theta \neq 0$. The Pauli matrices are a convenient basis to diagonalize $\hat{\mathbb{E}}_{\theta}$. It turns out that the eigenvalues and eigenvectors are given by:

$$
\begin{aligned}
\hat{\mathbb{E}}_{\theta}(\mathbb{1}) & =\mathbb{1}, \\
\hat{\mathbb{E}}_{\theta}\left(\sigma^{z}\right) & =\left(\sin ^{2} \theta-\cos ^{2} \theta\right) \sigma^{z}, \\
\hat{\mathbb{E}}_{\theta}\left(\sigma^{x} \pm \sigma^{y}\right) & =-\sin ^{2} \theta\left(\sigma^{x} \pm \sigma^{y}\right) .
\end{aligned}
$$

Clearly there is only one eigenvalue of modulus one and so the state $\omega_{\theta}$ is ergodic. Remark that for the AKLT model the three non-trivial eigenvalues $\hat{\mathbb{E}}$ coincide and are equal to $-\frac{1}{3}$. In view of (3.1) the correlations in AKLT state behave as $\left(-\frac{1}{3}\right)^{n}$.

(2) $\theta=0$. The minimal $\mathscr{B}$ is now two-dimensional. There are two eigenvalues of modulus 1:1 corresponding to the eigenvector $\mathbb{1}$ and -1 corresponding to the eigenvector $\sigma^{z}$. The state is ergodic but decomposes into two 2-periodic product states. These states are the ground states of the Ising antiferromagnet.

(3) $\theta=\pi / 2$. Because here the minimal $\mathscr{B}$ is one-dimensional, the state is a translation invariant product state and hence ergodic. It is even a pure state, indeed it is a product of pure states $A \in \mathscr{M}_{3} \mapsto\langle 0|A| 0\rangle$.

\section{Dilation Theory and Purely Generated States}

The aim of this section is to reduce general $C^{*}$-finitely correlated states to a particularly simple form, which will then be studied in more detail in the following sections. As a motivation, consider a $C^{*}$-finitely correlated state generated by $\left(\mathbb{E}, \rho, \mathbb{1}_{\mathscr{B}}\right)$, and suppose that $\mathbb{E}$ can be decomposed into a finite sum $\mathbb{E}=\sum_{x \in X} \mathbb{E}_{x}$ such that each $\mathbb{E}_{x}: \mathscr{A} \otimes \mathscr{B} \rightarrow \mathscr{B}$ is completely positive. Then with $\mathbb{E}_{x, A}(B) \equiv$ $\mathbb{E}_{x}(A \otimes B)$ we can define for all $i<j \in \mathbb{Z}$, and $x_{i}, \ldots, x_{j} \in X$ a positive linear functional $\omega_{i, j}\left[x_{i}, \ldots, x_{j}\right]$ on $\mathscr{A}_{\mathbb{Z}}$, such that for $n, m>0$,

$$
\begin{aligned}
& \omega_{i, j}\left[x_{i}, \ldots, x_{j}\right]\left(\left(A_{i-n} \otimes \cdots A_{i-1}\right) \otimes\left(A_{i} \otimes \cdots A_{j}\right) \otimes\left(A_{j+1} \otimes \cdots A_{j+m}\right)\right) \\
& \quad=\rho \circ\left(\mathbb{E}_{A_{i-n}} \circ \cdots \circ \mathbb{E}_{A_{i-1}}\right) \circ\left(\mathbb{E}_{x_{i}, A_{i}} \circ \cdots \circ \mathbb{E}_{x_{j}, A_{j}}\right) \circ\left(\mathbb{E}_{A_{j+1}} \circ \cdots \circ \mathbb{E}_{A_{j+m}}\right)(\mathbb{1}) .
\end{aligned}
$$

Clearly, the sum of these functionals over all choices of $x_{i}, \ldots, x_{j}$ is $\omega$. The normalization factors of these functionals define a cylinder measure $\mathbb{P}$ on the set $X^{\mathbb{Z}}$ of "paths" of a process over discrete "time" $\mathbb{Z}$ with state space $X$, i.e. with

$$
Z\left(x_{i}, \ldots, x_{j}\right)=\left\{\xi \in X^{\mathbb{Z}} \mid \xi_{t}=x_{t} \text { for } t=i, \ldots, j\right\}
$$

we have

$$
\omega_{i, j}\left[x_{i}, \ldots, x_{j}\right](\mathbb{1})=\mathbb{P}\left(Z\left(x_{i}, \ldots, x_{j}\right)\right)
$$


By increasing the interval $\{i, \ldots, j\}$ we obtain finer and finer decompositions of the state $\omega$. Using the theory of liftings [42] one can show that one can assign to each path $\xi \in X^{\mathbb{Z}}$ a state $\Omega[\xi]$ on $\mathscr{A}_{\mathbb{Z}}$, such that $\xi \mapsto \Omega[\xi](A)$ is cylinder measurable for each $A \in \mathscr{A}_{\mathbb{Z}}$, and

$$
\omega_{i, j}\left[x_{i}, \ldots, x_{j}\right](A)=\int_{\xi \in Z\left(x_{i}, \ldots, x_{j}\right)} \mathbb{P}(d \xi) \Omega[\xi](A) .
$$

In particular, $\omega=\int \mathbb{P}(d \xi) \Omega[\xi]$.

We can view the above construction as the introduction of a new set of "observables" to the system: in the refined description we can compute probabilities for the variables of the stochastic process $\left(\xi_{i}\right)_{i \in \mathbb{Z}}$ in addition to those of the original chain. A more straightforward way to introduce this refinement is to simply enlarge the one-site algebra $\mathscr{A}$, i.e. to use instead of $\mathscr{A}$ the algebra $\tilde{A}:=\mathscr{A} \otimes \mathscr{C}(X)$. A $C^{*}$-finitely correlated state $\tilde{\omega}$ on the chain $\tilde{A}_{\mathbb{Z}}$ is then generated by the completely positive map

$$
\tilde{\mathbb{E}}: \tilde{\mathscr{A}} \otimes \mathscr{B} \rightarrow \mathscr{B}:((A \otimes f) \otimes B) \mapsto \sum_{x \in X} f(x) \mathbb{E}_{x}(A \otimes B),
$$

and the same state $\rho$. Since $\tilde{\mathscr{A}}_{\mathbb{Z}}=(\mathscr{A} \otimes \mathscr{C}(X))_{\mathbb{Z}} \equiv \mathscr{A}_{\mathbb{Z}} \otimes \mathscr{C}(X)_{\mathbb{Z}} \equiv \mathscr{A}_{\mathbb{Z}} \otimes \mathscr{C}\left(X^{\mathbb{Z}}\right)$, the restriction of $\tilde{\omega}$ to $\mathscr{C}\left(X^{\mathbb{Z}}\right)$ defines a probability measure on $X^{\mathbb{Z}}$, which is just the $\mathbb{P}$ defined above. The integral decomposition of $\omega$ now simply becomes the direct integral decomposition of a state on a $C^{*}$-algebra of the form $\hat{\mathscr{A}} \otimes \mathscr{C}(\hat{X})$ for a compact space $\hat{X}$.

We shall now study the relation between decompositions of $\mathbb{E}$ and possible enlargements of the one-site algebra more systematically. We begin by defining those $\mathbb{E}$ for which no decomposition $\mathbb{E}=\sum_{x \in X} \mathbb{E}_{x}$ is possible.

4.1 Definition. A completely positive map is called pure, if it cannot be written as the sum of two completely positive maps, which are not proportional to itself. $A$ $C^{*}$-finitely correlated state $\omega$ on $\mathscr{A}_{\mathbb{Z}}$ is called purely generated, if it is generated by a pure map $\mathbb{E}: \mathscr{A} \otimes \mathscr{B} \rightarrow \mathscr{B}$.

Pure states in the usual sense are pure maps from an algebra into the one-dimensional algebra $\mathbb{C}$ in the sense of this definition. We note that, unlike for states, the pure unit preserving completely positive maps are in general only a small subclass of the extremal unit preserving completely positive maps.

4.2 Proposition. Let $\mathscr{A}$ be a finite-dimensional $C^{*}$-algebra, let $\omega$ be the state generated by $\mathbb{E}: \mathscr{A} \otimes \mathscr{B} \rightarrow \mathscr{B}$, and $\rho$, and assume that $\rho$ and the one-site restriction of $\omega$ are faithful on $\mathscr{A}$.

(1) Then $\omega$ is purely generated if and only if there are $d, k \in \mathbb{N}$ such that up to isomorphisms $\mathscr{A}=\mathscr{M}_{d}, \mathscr{B}=\mathscr{M}_{k}$, and $\mathbb{E}(A \otimes B)=V^{*}(A \otimes B) V$ for some isometry $V: \mathbb{C}^{k} \rightarrow \mathbb{C}^{d} \otimes \mathbb{C}^{k}$

(2) If $\mathscr{B}=\mathscr{M}_{k}$, then there is a faithful representation $\pi: \mathscr{A} \rightarrow \mathscr{B}(\mathscr{H})$ on a finite-dimensional Hilbert space $\mathscr{H}$, and a state $\tilde{\omega}$ on the chain $\mathscr{B}(\mathscr{H})_{\mathbb{Z}}$ generated by a pure map $\tilde{\mathbb{E}}: \mathscr{B}(\mathscr{H}) \otimes \mathscr{M}_{k} \rightarrow \mathscr{M}_{k}$ such that $\tilde{\mathbb{E}}(\mathbb{1} \otimes B)=\mathbb{E}(\mathbb{1} \otimes B)$ for all $B \in \mathscr{M}_{k}$, and

$$
\omega\left(A_{i} \otimes \cdots A_{i+n}\right)=\tilde{\omega}\left(\pi\left(A_{i}\right) \otimes \cdots \pi\left(A_{i+n}\right)\right) .
$$


Proof. Each finite-dimensional $C^{*}$-algebra is the direct sum of matrix algebras. A completely positive map between direct sums $T: \bigoplus_{i} \mathscr{A}_{i} \rightarrow \bigoplus_{j} \mathscr{B}_{j}$ has a natural decomposition into completely positive summands $T_{i j}$. Hence such a map can only be pure if it is supported by a single summand $\mathscr{A}_{i}$, and maps into a single summand $\mathscr{B}_{j}$. By the non-degeneracy condition we conclude that both $\mathscr{A}$ and $\mathscr{B}$ must be irreducible matrix algebras if $\mathbb{E}$ is pure. In particular, we may suppose in both parts of the proposition that $\mathscr{B}=\mathscr{M}_{k}$.

Consider now the Stinespring dilation [59] of $\mathbb{E}$. This yields a representation $\tilde{\pi}: \mathscr{A} \otimes \mathscr{B} \rightarrow \mathscr{B}(\tilde{\mathscr{H}})$ on a Hilbert space $\tilde{\mathscr{H}}$, and an isometry $V: \mathbb{C}^{k} \rightarrow \tilde{\mathscr{H}}$ such that $\mathbb{E}(X)=V^{*} \tilde{\pi}(X) V$ for all $X \in \mathscr{A} \otimes \mathscr{B}$. Since $\tilde{\pi}\left(\mathbb{1}_{\mathscr{A}} \otimes \mathscr{B}\right)$ is a copy of the $k \times k$-matrices

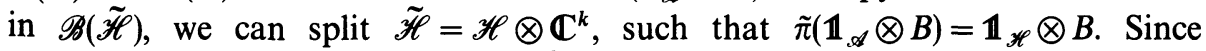
$\tilde{\pi}\left(\mathscr{A} \otimes \mathbb{1}_{\mathscr{B}}\right)$ commutes with $\mathbb{1}_{\mathscr{H}} \otimes \mathscr{B}\left(\mathbb{C}^{k}\right)$, it is clear that we also have a representation $\pi: \mathscr{A} \rightarrow \mathscr{B}(\mathscr{H})$ with $\tilde{\pi}\left(A \otimes \mathbb{1}_{\mathscr{B}}\right)=\pi(A) \otimes \mathbb{1}$.

It is a basic property of the Stinespring dilation that completely positive decompositions $\mathbb{E}=\mathbb{E}_{1}+\mathbb{E}_{2}$ are in one-to-one correspondence to the positive elements in the commutant of $\tilde{\pi}$, i.e. to elements of the form $E_{1} \otimes \mathbb{1}$ with $[E, \pi(\mathscr{A})]=\{0\}$. The correspondence is given by $\mathbb{E}_{1}(A \otimes B)=V^{*}(E \otimes \mathbb{1})(\pi(A) \otimes B) V$. Hence $\mathbb{E}$ is pure iff the representation $\pi$ is irreducible. This is the case iff we can identify $\mathscr{H}$ with $\mathbb{C}^{d}$ and $\pi$ with the identity representation. This proves (1). The claim (2) follows by straightforward computation with the objects obtained from the dilation and the $C^{*}$-finitely correlated state $\tilde{\omega}$ they generate on the chain $\mathscr{B}(\mathscr{H})_{\mathbf{Z}}$.

Note that by Lemma 2.5 the condition $\mathscr{B}=\mathscr{M}_{k}$ is not a restriction on the state $\omega$. The "additional variables" $x \in X$ discussed in the beginning of this section now correspond to the commutant of $\pi(\mathscr{A})$ in $\mathscr{B}(\mathscr{H})$. This algebra is non-abelian, and it describes all possible extensions by abelian algebras $\mathscr{C}(X)$ simultaneously. This is a typical feature of all applications of the Stinespring dilation. It would therefore be appropriate to call the purely generated state $\tilde{\omega}$ the dilation of $\omega$. Note, however, that we do not assert the uniqueness of the representation of a $C^{*}$-finitely correlated state in terms of $(\mathbb{E}, \rho, e)$, and that the state $\tilde{\omega}$ depends on these objects as well. Since $\pi$ in Proposition 4.2(2) is a faithful homomorphism, we may consider $\mathscr{A}$ as a subalgbra of $\mathscr{B}(\mathscr{H})$, so that every $C^{*}$-finitely correlated state arises from a purely generated state by restricting to a subalgebra of the one-site algebra. In classical probability a faithful homomorphism $\pi: \mathscr{C}(Y) \rightarrow \mathscr{C}(X)$ implies the existence of a surjective map $\pi_{*}: X \rightarrow Y$ with $\pi f(x)=f\left(\pi_{*} x\right)$. Thus the variables $y \in Y$ are functions on $X$. Extending this analogy to the non-commutative setting we can interpret the above proposition by saying that every $C^{*}$-finitely correlated state is a "local function" of a purely generated $C^{*}$-finitely correlated state.

Since Proposition 3.1(3) gives a criterion for the ergodicity of $\omega$ which depends only on $\hat{\mathbb{E}}$, it is clear that the purely generated state $\tilde{\omega}$ associated with $\omega$ by this proposition will be ergodic whenever $\omega$ is. Similarly, if translation symmetry is not broken in $\omega$, i.e. if the peripheral spectrum of $\hat{\mathbb{E}}$ consists only of the simple eigenvalue 1 , the same will be the case for $\tilde{\omega}$. We therefore arrive to the following procedure for studying a general $C^{*}$-finitely correlated state: by applying Corollary 3.4 we first decompose the state into its unique extremal periodic components, which are $C^{*}$-finitely correlated states with the additional property 
that $\hat{\mathbb{E}}^{n}$ converges to $\hat{\mathbb{E}}^{\infty}(B)=\rho(B) \mathbb{1}_{\mathscr{B}}$ exponentially fast. Then, by applying Proposition 4.2(1) we associate with each component a purely generated state with the same property. Thus purely generated states with strictly contracting $\hat{\mathbb{E}}$ are the basic building blocks for all $C^{*}$-finitely correlated states, and will be studied in detail in the following two sections.

Using Proposition 4.2 we can now give a simple proof of the remainder of Proposition 2.7.

Proof of 2.7. It remains to be shown that every $C^{*}$-finitely correlated state admits a valence bond representation with the special properties listed in the proposition. It is evident that it suffices to construct a valence bond representation for the dilation $\tilde{\omega}$ of the given state. Thus we may assume by Proposition 4.2(1) that $\mathscr{A}=\mathscr{M}_{d} \equiv \mathscr{B}(\mathscr{H}), \mathscr{B}=\mathscr{M}_{k} \equiv \mathscr{B}(\mathscr{K})$, and $\mathbb{E}(A \otimes B)=V^{*}(A \otimes B) V$ for some isometry $V: \mathscr{K} \rightarrow \mathscr{H} \otimes \mathscr{K}$, and that $\rho$ is faithful. We can therefore write $\rho(B)=\sum_{\alpha} \rho_{\alpha}\left\langle\chi_{\alpha}, B \chi_{\alpha}\right\rangle$ for some orthonormal basis $\left\{\chi_{\alpha}\right\}_{\alpha=1}^{k} \subset \mathscr{K}$. We shall then define the objects in the valence bond construction as follows: $\overline{\mathscr{B}}=\mathscr{B}(\overline{\mathscr{K}})$ will be the algebra of operators on the conjugate Hilbert space $\overline{\mathscr{K}}$, i.e. on a space of the same dimension $k$ as $\mathscr{K}$, which is connected with $\mathscr{K}$ via some anti-unitary operator $\chi \mapsto \bar{\chi}$. The state $\Phi: \overline{\mathscr{B}} \otimes \mathscr{B} \rightarrow \mathbb{C}$ will be pure, and its restriction to $\mathscr{B}$ will be just the faithful state $\rho$. We set

$$
\Phi(X)=\langle\varphi, X \varphi\rangle \quad \text { with } \quad \varphi=\sum_{\alpha} \sqrt{\rho_{\alpha} \chi_{\alpha}} \otimes \chi_{\alpha} \in \overline{\mathscr{K}} \otimes \mathscr{K} .
$$

(Thus $B \mapsto \mathbb{1}_{\overline{\mathscr{B}}} \otimes B$ is just the GNS-representation of $(\mathscr{B}, \rho)$ with cyclic vector $\varphi$ ). The map $\mathbb{F}$ is defined in terms of its Stinespring dilation $\hat{V}: \overline{\mathscr{K}} \otimes \mathscr{K} \rightarrow \mathscr{H}$ by

$$
\mathbb{F}(A)=\hat{V}^{*} A \hat{V} \quad \text { with }\left\langle\psi, \hat{V} \chi \otimes \overline{\chi^{\prime}}\right\rangle=\left\langle\psi \otimes\left(\rho^{-1 / 2} \chi^{\prime}\right), V \chi\right\rangle .
$$

In order to complete the proof we have to check the compatibility conditions for $\mathbb{F}$ and $\Phi$, which ensure that $\mathbb{F}, \Phi$ define a valence bond state, as well as the two equations used in the proof of the trivial direction to show that this valence bond state coincides with $\omega$. One of the latter equations, namely $\rho(B)=\Phi\left(\mathbb{1}_{\bar{B}} \otimes B\right)$ has already been noted above. We check equation $\mathbb{E}(A \otimes B)=\left(\operatorname{id}_{\mathscr{B}} \otimes \Phi\right)(\mathbb{F}(A) \otimes B)$ by taking matrix elements, using a basis $\left\{\psi_{\mu}\right\}_{\mu=1}^{d} \subset \mathscr{H}$ :

$$
\begin{aligned}
\langle\chi, & \left.\left(\operatorname{id}_{\mathscr{B}} \otimes \Phi\right)(\mathbb{F}(A) \otimes B) \chi^{\prime}\right\rangle \\
= & \sum_{\alpha, \beta} \sqrt{\rho_{\alpha} \rho_{\beta}}\left\langle\chi \otimes \bar{\chi}_{\alpha} \otimes \chi_{\alpha},(\mathbb{F}(A) \otimes B) \chi^{\prime} \otimes \bar{\chi}_{\beta} \otimes \chi_{\beta}\right\rangle \\
= & \sum_{\alpha, \beta} \sqrt{\rho_{\alpha} \rho_{\beta}}\left\langle\hat{V} \chi \otimes \bar{\chi}_{\alpha}, A \hat{V} \chi^{\prime} \otimes \bar{\chi}_{\beta}\right\rangle\left\langle\chi_{\alpha}, B \chi_{\beta}\right\rangle \\
= & \sum_{\alpha, \beta, \mu, v} \sqrt{\rho_{\alpha} \rho_{\beta}}\left\langle\hat{V} \chi \otimes \bar{\chi}_{\alpha}, \psi_{\mu}\right\rangle\left\langle\psi_{\mu}, A \psi_{v}\right\rangle\left\langle\psi_{v}, \hat{V} \chi^{\prime} \otimes \bar{\chi}_{\beta}\right\rangle\left\langle\chi_{\alpha}, B \chi_{\beta}\right\rangle \\
= & \sum_{\alpha, \beta, \mu, v}\left\langle V \chi, \psi_{\mu} \otimes \chi_{\alpha}\right\rangle\left\langle\psi_{\mu} \otimes \chi_{\alpha},(A \otimes B) \psi_{v} \otimes \chi_{\beta}\right\rangle\left\langle\psi_{v} \otimes \chi_{\beta}, V \chi^{\prime}\right\rangle \\
= & \left\langle V \chi,(A \otimes B) V \chi^{\prime}\right\rangle=\left\langle\chi, \mathbb{E}(A \otimes B) \chi^{\prime}\right\rangle .
\end{aligned}
$$

This immediately implies the compatibility condition for $\mathbb{1}_{\mathscr{B}}$. To demonstrate the 
other condition we proceed similarly:

$$
\begin{aligned}
& \left\langle\chi,\left(\Phi \otimes \operatorname{id}_{\overline{\mathscr{B}}}\right)\left(\mathbb{1}_{\overline{\mathscr{B}}} \otimes \mathbb{F}(\mathbb{1})\right) \chi^{\prime}\right\rangle \\
& =\sum_{\alpha, \beta} \sqrt{\rho_{\alpha} \rho_{\beta}}\left\langle\overline{\chi_{\alpha}} \otimes \chi_{\alpha} \otimes \chi,\left(\mathbb{1}_{\overline{\mathscr{B}}} \otimes \mathbb{F}(\mathbb{1})\right) \overline{\chi_{\beta}} \otimes \chi_{\beta} \otimes \chi^{\prime}\right\rangle \\
& =\sum_{\alpha} \rho_{\alpha}\left\langle\chi_{\alpha} \otimes \chi, \mathbb{F}(\mathbb{1}) \chi_{\alpha} \otimes \chi^{\prime}\right\rangle \\
& =\sum_{\alpha, \mu, \nu} \rho_{\alpha}\left\langle\hat{V} \chi_{\alpha} \otimes \chi, \psi_{\mu}\right\rangle\left\langle\psi_{\mu}, \mathbb{1}_{\overline{\mathscr{B}}} \psi_{v}\right\rangle\left\langle\psi_{\mu}, \hat{V} \chi_{\alpha} \otimes \chi^{\prime}\right\rangle \\
& =\sum_{\alpha, \mu} \rho_{\alpha}\left\langle V \chi_{\alpha}, \psi_{\mu} \otimes \rho^{-1 / 2} \bar{\chi}\right\rangle\left\langle\psi_{\mu} \otimes \rho^{-1 / 2} \overline{\chi^{\prime}}, V \chi_{\alpha}\right\rangle \\
& =\operatorname{Tr}\left(\rho \mathbb{E}\left(\mathbb{1} \otimes\left(\rho^{-1 / 2}|\bar{\chi}\rangle\left\langle\overline{\chi^{\prime}}\right| \rho^{-1 / 2}\right)\right)\right. \\
& =\operatorname{Tr}\left(\rho \rho^{-1 / 2}|\bar{\chi}\rangle\left\langle\overline{\chi^{\prime}}\right| \rho^{-1 / 2}\right)=\left\langle\overline{\chi^{\prime}}, \bar{\chi}\right\rangle=\left\langle\chi, \chi^{\prime}\right\rangle
\end{aligned}
$$

by the compatibility condition for $\mathbb{E}$ and $\rho$.

It is clear from this proof that for purely generated states the completely positive map $\mathbb{F}$ will also be pure. The scheme for defining valence bond states can then be transformed into a scheme for maps between Hilbert spaces, with $\mathbb{F}$ replaced by $\hat{V}, \Phi$ replaced by the map $\lambda \in \mathbb{C} \mapsto \lambda \cdot \varphi \in \overline{\mathscr{K}} \otimes \mathscr{K}$, and all arrows are reversed.

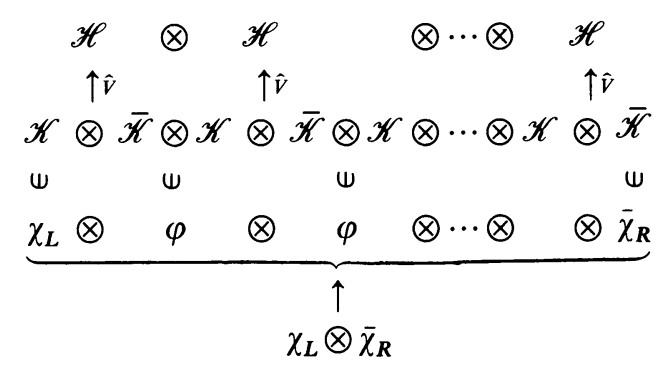

Fig. 2. Definition of the map $\Gamma_{n}: \mathscr{K} \otimes \overline{\mathscr{K}} \rightarrow \mathscr{H}^{\otimes n}$

The map $\Gamma_{n}: \mathscr{K} \otimes \overline{\mathscr{K}} \rightarrow \mathscr{H}^{\otimes n}$ depicted in this diagram will play an important part in the next two sections. (Compare the algebraic definition of $\Gamma_{n}$ in Eq. (5.5)). In the literature $[7,18,17,19-21]$ valence bond states have usually been discussed in terms of the vectors $\Gamma_{n}\left(\chi_{L} \otimes \bar{\chi}_{R}\right)$. This approach has the disadvantage that it yields a state on the infinite chain only in the limit $n \rightarrow \infty$. This limit need not exist, i.e. there may be different accumulation points of the sequence of $n$-particle states, depending on the choice of $\chi_{L, n}$, and $\chi_{R, n}$. In contrast, we can work with an explicit expression for (the $n$-site restriction) of the state $\omega$ from the beginning, and even in the non-ergodic situation, we have an explicit parametrization of the translation invariant limit points by the 1-eigenspace of $\hat{\mathbb{E}}$.

\section{Ground State Property of Purely Generated States}

In this section we shall begin a more detailed study of the states, which were identified as the basic building blocks for all $C^{*}$-finitely correlated states in Sect. 4 , 
namely the purely generated states which cannot be further decomposed into periodic states. By Proposition 4.2(1) we can therefore take $\mathscr{A}=\mathscr{M}_{d}$ and $\mathscr{B}=\mathscr{M}_{k}$ as the algebras of $d \times d$ - and $k \times k$-matrices, and take them to be represented on Hilbert spaces $\mathscr{H}, \mathscr{K}$ of dimensions $d, k$, respectively. Moreover, the pure map $\mathbb{E}: \mathscr{A} \otimes \mathscr{B} \rightarrow \mathscr{B}$ is of the form $\mathbb{E}(A \otimes B)=V^{*}(A \otimes B) V$ for some isometry $V: \mathscr{K} \rightarrow \mathscr{H} \otimes \mathscr{K}$. The property that translation symmetry is not broken, or, equivalently, that $\hat{\mathbb{E}}$ has trivial peripheral spectrum, can be expressed as

$$
\lim _{n \rightarrow \infty} \hat{\mathbb{E}}^{n}(B) \equiv \hat{\mathbb{E}}^{\infty}(B)=\operatorname{Tr}(\rho B) \cdot \mathbb{1}_{\mathscr{B}}
$$

for all $B \in \mathscr{B}$, where $\rho$ is the non-singular density matrix invariant under $\hat{\mathbb{E}}$. (Here we abuse notation, writing $\rho(B)=\operatorname{Tr}(\rho B)$.) Note that $\mathbb{E}$, and hence $\rho$ are both determined by $V$, so the state $\omega$ is completely specified by this isometry.

Before entering into the study of this manifold of states, it may be useful to give a rough estimate of its dimension. For fixed $d, k$ we have to study the set of isometries $V: \mathbb{C}^{k} \rightarrow \mathbb{C}^{d} \otimes \mathbb{C}^{k}$. Starting from the given isometry $V_{0}$ we get all others in the form $V=U V_{0} U^{\prime}$ with unitaries $U \in \mathscr{B}\left(\mathbb{C}^{d} \otimes \mathbb{C}^{k}\right)$, and $U^{\prime} \in \mathscr{M}_{k}$. The transformation $V=\left(\mathbb{1} \otimes U^{\prime *}\right) V_{0} U^{\prime}$ corresponds to a change of basis in $\mathbb{C}^{k}$, hence does not change $\omega$. Thus we only have to consider $V=U V_{0}$. Then $U_{1} V_{0}=U_{2} V_{0}$ iff $U_{1}^{*} U_{2} V_{0}=V_{0}$, i.e. if the projection $V_{0} V_{0}^{*}$ reduces $U_{1}^{*} U_{2}$, and $U_{1}^{*} U_{2}$ is determined by an arbitrary unitary operator in the complement of $V_{0} \mathbb{C}^{k}$. Since the unitary group in $\mathbb{C}^{d} \otimes \mathbb{C}^{k}$ is a manifold of dimension $d^{2} k^{2}$, and the unitaries $U$ yielding the same isometry are parametrized by the unitary group on a $(d k-k)$-dimensional space, we find a manifold of isometries of dimension $k^{2}(2 d-1)$. From this we have to subtract one, since isometries differing by a phase yield the same $\mathbb{E}$. For example, the state of [5], which is also studied in Sect. 7.4 below $(d=3, k=2)$, is therefore embedded into a 4.5-1=19-dimensional manifold.

It will be convenient to choose bases $\left\{\psi_{\mu}\right\}_{\mu=1}^{d} \subset \mathscr{H}$ and $\left\{\chi_{\alpha}\right\}_{\alpha=1}^{k} \subset \mathscr{K}$. This determines matrices $v(\mu) \in \mathscr{B}$ such that

$$
V \chi=\sum_{\mu} \psi_{\mu} \otimes\left(v(\mu)^{*} \chi\right)
$$

In a more basis-free spirit we could also define a linear map $\bar{v}: \mathscr{H} \rightarrow \mathscr{B}$ by $\left\langle\chi, \bar{v}(\psi) \chi^{\prime}\right\rangle=\left\langle V \chi, \psi \otimes \chi^{\prime}\right\rangle$, so that $v(\mu)=\bar{v}\left(\psi_{\mu}\right)$. However, some of the equations become more transparent in a fixed basis. The following are obtained by considering the general matrix element $\left\langle\chi, \mathbb{E}(A \otimes B) \chi^{\prime}\right\rangle$,

$$
\begin{aligned}
\mathbb{E}_{A}(B) \equiv \mathbb{E}(A \otimes B) & =\sum_{\mu, v}\left\langle\psi_{\mu}, A \psi_{v}\right\rangle v(\mu) B v(v)^{*}, \\
\sum_{\mu} v(\mu) v(\mu)^{*} & =\mathbb{E}(\mathbb{1} \otimes \mathbb{1})=\mathbb{1}, \\
\sum_{\mu} v(\mu)^{*} \rho v(\mu) & =\rho, \\
\hat{\mathbb{E}}(B) & =\sum_{\mu} v(\mu) B v(\mu)^{*} .
\end{aligned}
$$


The advantage of writing $V$ and $\mathbb{E}$ in this form is that these formulas are easily generalized to longer segments of the chain. We merely have to iterate (5.3a). This gives

$$
\begin{aligned}
\mathbb{E}_{A_{1} \otimes \cdots A_{n}}^{(n)}= & \mathbb{E}_{A_{1}} \circ \cdots \circ \mathbb{E}_{A_{n}}(B) \\
= & \sum_{\substack{\mu_{1}, \ldots, \mu_{n} \\
v_{1}, \cdots, v_{n}}}\left\langle\psi_{\mu_{1}} \otimes \cdots \psi_{\mu_{n}}, A_{1} \otimes \cdots A_{n} \psi_{v_{1}} \otimes \cdots \psi_{v_{n}}\right\rangle \\
& \cdot v\left(\mu_{1}\right) \cdots v\left(\mu_{n}\right) B v\left(v_{n}\right)^{*} \cdots v\left(v_{1}\right)^{*} .
\end{aligned}
$$

This formula has exactly the same structure as (5.3a), with $\left\{\psi_{\mu}\right\}_{\mu=1}^{d}$ replaced by the corresponding product basis $\left\{\psi_{\mu_{1}, \ldots, \mu_{n}}=\psi_{\mu_{1}} \otimes \cdots \psi_{\mu_{n}}\right\} \subset \mathscr{H}^{\otimes n}$, and $v\left(\mu_{1}, \ldots, \mu_{n}\right)=$ $v\left(\mu_{1}\right) \cdots v\left(\mu_{n}\right) \in \mathscr{A}$.

Using this notation, we can give a more useful expression for the map $\Gamma_{n}: \mathscr{K} \otimes \overline{\mathscr{K}} \rightarrow \mathscr{H}^{\otimes n}$ described at the end of Sect. 4. For the purposes of this section it will be better to use the natural identification of $\mathscr{K} \otimes \mathscr{K}$ with $\mathscr{M}_{k} \equiv \mathscr{B}$. Then $\Gamma_{n}$ becomes a map $\Gamma_{n}: \mathscr{B} \rightarrow \mathscr{H}^{\otimes n}$, with

$$
\Gamma_{n}(B)=\sum_{\mu_{1}, \ldots, \mu_{n}} \psi_{\mu_{1}} \otimes \cdots \psi_{\mu_{n}} \operatorname{Tr}\left(B v\left(\mu_{n}\right)^{*} \cdots v\left(\mu_{1}\right)^{*}\right) .
$$

We shall only use this definition in the sequel and leave it to the reader to check that this indeed coincides with the map introduced in Sect. 4. Note that Eq. (5.5) can be written simply as the corresponding expression for $n=1$, when $\mu_{1}$ is replaced by the tuple $\left(\mu_{1}, \ldots, \mu_{n}\right)$. Therefore it suffices in the proof of some algebraic relations involving $\Gamma_{n}$ to take $n=1$. In Sect. 4 the range of $\Gamma_{n}$ was described as the set of valence bond vectors associated with the state $\omega$. We shall denote this range by $\mathscr{G}_{n}=\Gamma_{n}(\mathscr{B}) \subset \mathscr{H}^{\otimes n}$, and the corresponding orthogonal projection by $G_{n}$.

This suggests that the $n$-step restriction of $\omega$ will be supported by $\mathscr{G}_{n}$. Here we prove a more detailed result, giving an alternative formula for $\omega$ in terms of $\Gamma_{n}$, and a fixed density matrix $W_{\infty}$ on $\mathscr{B}$, where $\mathscr{B}$ is considered as Hilbert space with the inner product

$$
\langle A, B\rangle_{\rho}:=\operatorname{Tr}\left(\rho A^{*} B\right) .
$$

Since $W_{\infty}$ is given by an invertible linear transformation, the formula below also shows that the support of the $n$-step restriction is, in fact, equal to $G_{n}$. The Lemma also gives a formula for matrix elements between valence bond states, which will be useful below.

\subsection{Lemma}

(1) For all $A \in \mathscr{A}^{\otimes n}$,

$$
\omega(A)=\operatorname{Tr}\left(\Gamma_{n} W_{\infty} \Gamma_{n}^{*} \cdot A\right),
$$

where $W_{\infty}: \mathscr{B} \rightarrow \mathscr{B}$ is the density matrix on $\left(\mathscr{B},\langle\cdot, \cdot\rangle_{\rho}\right)$ with $W_{\infty}(B)=\rho B p$.

(2) For all $A \in \mathscr{A}{ }^{\otimes n}$, and $B, C \in \mathscr{B}$,

$$
\left\langle\Gamma_{n}(B), A \Gamma_{n}(C)\right\rangle=\sum_{\alpha, \beta}\left\langle\chi_{\alpha}, \mathbb{E}_{A}^{(n)}\left(B^{*}\left|\chi_{\alpha}\right\rangle\left\langle\chi_{\beta}\right| C\right) \chi_{\beta}\right\rangle .
$$


Proof. We need to prove only the case $n=1$, from which the general case follows by substituting $n$-tuples for $\mu, v$. From (5.3) we get

$$
\begin{aligned}
\omega(A) & =\sum_{\mu, v}\left\langle\psi_{\mu}, A \psi_{v}\right\rangle \operatorname{Tr}\left(\rho v(\mu) v(v)^{*}\right) \\
& =\sum_{\mu, v, \alpha, \beta}\left\langle\psi_{\mu}, A \psi_{v}\right\rangle\left\langle\chi_{\alpha}, \sqrt{\rho} v(\mu) \chi_{\beta}\right\rangle\left\langle\chi_{\beta}, v(v)^{*} \sqrt{\rho} \chi_{\alpha}\right\rangle \\
& =\sum_{\alpha, \beta}\left\langle\Gamma_{1}\left(B_{\alpha \beta}\right), A \Gamma_{1}\left(B_{\alpha \beta}\right)\right\rangle
\end{aligned}
$$

where $B_{\alpha \beta}=\left|\sqrt{\rho} \chi_{\alpha}\right\rangle\left\langle\chi_{\beta}\right|$. Hence (5.7) holds with $W_{\infty}$ determined from

$$
\begin{aligned}
\left\langle C, W_{\infty} C^{\prime}\right\rangle_{\rho} & =\sum_{\alpha, \beta}\left\langle B_{\alpha \beta}, C^{\prime}\right\rangle_{\rho}\left\langle C, B_{\alpha \beta}\right\rangle_{\rho} \\
& =\sum_{\alpha, \beta} \operatorname{Tr}\left(\rho\left|\chi_{\beta}\right\rangle\left\langle\chi_{\alpha}\right| \sqrt{\rho} C^{\prime}\right) \operatorname{Tr}\left(\rho C^{*} \sqrt{\rho}\left|\chi_{\alpha}\right\rangle\left\langle\chi_{\beta}\right|\right) \\
& =\operatorname{Tr}\left(\rho C^{*} \sqrt{\rho} \sqrt{\rho} C^{\prime} \rho\right)=\left\langle C, \rho C^{\prime} \rho\right\rangle_{\rho} .
\end{aligned}
$$

This proves (1). For part (2) we write out the traces in the definition of $\Gamma$ with respect to the basis $\left\{\chi_{\alpha}\right\}$ :

$$
\begin{aligned}
\left\langle\Gamma_{1}(B), A \Gamma_{1}(C)\right\rangle & =\sum_{\alpha \beta, \mu \nu}\left\langle\psi_{\mu}, A \psi_{v}\right\rangle\left\langle\chi_{\alpha}, v(\mu) B^{*} \chi_{\alpha}\right\rangle\left\langle\chi_{\beta}, C v(v)^{*} \chi_{\beta}\right\rangle \\
& =\sum_{\alpha \beta}\left\langle\chi_{\alpha}, \mathbb{E}_{A}\left(B^{*}\left|\chi_{\alpha}\right\rangle\left\langle\chi_{\beta}\right| C\right) \chi_{\beta}\right\rangle .
\end{aligned}
$$

For large $n$ scalar products involving valence bond vectors can be evaluated by using the strict contraction property (5.1). The following lemma gives two basic estimates of this kind.

5.2 Lemma. Let $\lambda$ be such that $\left|\lambda_{i}\right|<\lambda<1$ for all eigenvalues $\lambda_{i}$ of $\hat{\mathbb{E}}$ different from 1. Then there is a constant $c$ such that for all $n$ :

$$
a(n):=\operatorname{Tr}\left(\rho^{-1}\right)\left\|\hat{\mathbb{E}}^{n}-\hat{\mathbb{E}}^{\infty}\right\| \leqq c \lambda^{n} .
$$

Moreover, we have the following estimates:

(1) For all $B, C \in \mathscr{B}$ :

$$
\left|\left\langle\Gamma_{n}(B), \Gamma_{n}(C)\right\rangle-\langle B, C\rangle_{\rho}\right| \leqq a(n)\|B\|_{\rho} \cdot\|C\|_{\rho} .
$$

(2) For $A \in \mathscr{A}^{\otimes m}, \ell, r \in \mathbb{N}$, and $B, C \in \mathscr{B}$ :

$$
\begin{aligned}
& \left|\left\langle\Gamma_{\ell+m+r}(B),\left(\mathbb{1}_{\ell} \otimes A \otimes \mathbb{1}_{r}\right) \Gamma_{\ell+m+r}(C)\right\rangle-\omega(A)\langle B, C\rangle_{\rho}\right| \\
& \quad \leqq(a(\ell)+a(r))\|A\| \cdot\|B\|_{\rho} \cdot\|C\|_{\rho} .
\end{aligned}
$$

Proof. (1) Applying (5.8) with $A=\mathbb{1}$ we get

$$
\left\langle\Gamma_{n}(B), \Gamma_{n}(C)\right\rangle=\sum_{\alpha, \beta}\left\langle\chi_{\alpha}, \hat{\mathbb{E}}^{n}\left(B^{*}\left|\chi_{\alpha}\right\rangle\left\langle\chi_{\beta}\right| C\right) \chi_{\beta}\right\rangle .
$$

Replacing $\hat{\mathbb{E}}^{n}$ by $\hat{\mathbb{E}}^{\infty}$ in the last expression we obtain

$$
\sum_{\alpha, \beta}\left\langle\chi_{\alpha}, \chi_{\beta}\right\rangle \operatorname{Tr}\left(\rho B^{*}\left|\chi_{\alpha}\right\rangle\left\langle\chi_{\beta}\right| C\right)=\operatorname{Tr}\left(\rho B^{*} C\right)=\langle B, C\rangle_{\rho} .
$$


The difference is less than

$$
\begin{aligned}
& \sum_{\alpha, \beta}\left\|\chi_{\alpha}\right\| \cdot\left\|\hat{\mathbb{E}}^{n}-\hat{\mathbb{E}}^{\infty}\right\| \cdot\left\|B^{*} \chi_{\alpha}\right\| \cdot\left\|C^{*} \chi_{\beta}\right\| \cdot\left\|\chi_{\beta}\right\| \\
& \leqq\left\|\hat{\mathbb{E}}^{n}-\hat{\mathbb{E}}^{\infty}\right\|\left(\sum_{\alpha}\left\|B^{*} \chi_{\alpha}\right\|\right)\left(\sum_{\beta}\left\|C^{*} \chi_{\beta}\right\|\right) \\
& \leqq\left\|\hat{\mathbb{E}}^{n}-\hat{\mathbb{E}}^{\infty}\right\| \cdot \operatorname{Tr}\left(\rho^{-1}\right)\|B\|_{\rho} \cdot\|C\|_{\rho},
\end{aligned}
$$

where at the last inequality we have used a special basis $\left\{\chi_{\alpha}\right\}$ with $\rho=\sum_{\alpha} \rho_{\alpha}\left|\chi_{\alpha}\right\rangle\left\langle\chi_{\alpha}\right|$ to obtain

$$
\begin{aligned}
\left(\sum_{\alpha}\left\|B^{*} \chi_{\alpha}\right\|\right)^{2} & =\left(\sum_{\alpha}\left\|B^{*} \chi_{\alpha}\right\| \rho_{\alpha}^{1 / 2} \cdot \rho_{\alpha}^{-1 / 2}\right)^{2} \\
& \leqq\left(\sum_{\alpha} \rho_{\alpha}\left\langle\chi_{\alpha}, B B^{*} \chi_{\alpha}\right\rangle\right) \sum_{\alpha} \rho_{\alpha}^{-1}=\|B\|_{\rho}^{2} \operatorname{Tr}\left(\rho^{-1}\right) .
\end{aligned}
$$

(2) Again by 5.8 we have

$$
\left\langle\Gamma_{\ell+m+r}(B),\left(\mathbb{1}_{\ell} \otimes A \otimes \mathbb{1}_{r}\right) \Gamma_{\ell+m+r}(C)\right\rangle=\sum_{\alpha, \beta}\left\langle\chi_{\alpha}, \hat{\mathbb{E}}^{\ell} \mathbb{E}_{A}^{(m)} \hat{\mathbb{E}}^{r}\left(B^{*}\left|\chi_{\alpha}\right\rangle\left\langle\chi_{\beta}\right| C\right) \chi_{\beta}\right\rangle .
$$

Writing the product of the three $\mathbb{E}$-operators as

$$
\hat{\mathbb{E}}^{\infty} \mathbb{E}_{A}^{(m)} \hat{\mathbb{E}}^{\infty}+\hat{\mathbb{E}}^{\ell} \mathbb{E}_{A}^{(m)}\left(\hat{\mathbb{E}}^{r}-\hat{\mathbb{E}}^{\infty}\right)+\left(\hat{\mathbb{E}}^{\ell}-\hat{\mathbb{E}}^{\infty}\right) \mathbb{E}_{A}^{(m)} \hat{\mathbb{E}}^{\infty},
$$

we obtain the leading term

$$
\sum_{\alpha} \operatorname{Tr}\left(\rho \hat{\mathbb{E}}_{A}^{(m)}(\mathbb{1})\right) \operatorname{Tr}\left(\rho B^{*}\left|\chi_{\alpha}\right\rangle\left\langle\chi_{\alpha}\right| C\right)=\omega(A)\langle B, C\rangle_{\rho}
$$

and two remainder terms, which are estimated exactly as in (1).

As a consequence of Lemma 5.2(1), the maps $\Gamma_{n}$ are injective for all sufficiently large $n$. However, the bound given does not exclude that this property holds sporadically for some small $n$, but fails for some larger $n^{\prime}$ before becoming valid universally. The following lemma excludes this possibility by showing a quantity to be monotone, which vanishes iff $\Gamma_{n}$ is not injective.

\subsection{Lemma. The quantity}

$$
a_{-}(n)=\inf \operatorname{spec}\left(\Gamma_{n}^{*} \Gamma_{n}\right):=\inf _{B \neq 0} \frac{\left\|\Gamma_{n}(B)\right\|^{2}}{\|B\|_{\rho}^{2}} \geqq 1-a(n)
$$

is non-decreasing in $n$.

Proof. Since $\left(\left\|\Gamma_{n}(B)\right\|^{2}-\|B\|_{\rho}^{2}\right) \geqq-a(n)\|B\|_{\rho}^{2}$, it is clear that $a_{-}(n) \geqq 1-a(n)$. The monotonicity of $a_{-}$follows from the estimate

$$
\begin{aligned}
\left\|\Gamma_{n+1}(B)\right\|^{2} & =\sum_{\mu_{n+1}} \sum_{\mu_{1}, \ldots, \mu_{n}}\left|\operatorname{Tr}\left(\left(B v\left(\mu_{n+1}\right)^{*}\right) v\left(\mu_{n}\right)^{*} \cdots v\left(\mu_{1}\right)^{*}\right)\right|^{2} \\
& \geqq \sum_{\mu_{n+1}} a_{-}(n) \operatorname{Tr}\left(\rho\left(B v\left(\mu_{n+1}\right)^{*}\right)^{*}\left(B v\left(\mu_{n+1}\right)^{*}\right)\right)
\end{aligned}
$$




$$
\begin{aligned}
& =a_{-}(n) \operatorname{Tr}\left(\rho \sum_{\mu} v(\mu) B^{*} B v(\mu)^{*}\right) \\
& =a_{-}(n) \operatorname{Tr}\left(\rho \hat{\mathbb{E}}\left(B^{*} B\right)\right)=a_{-}(n) \operatorname{Tr}\left(\rho B^{*} B\right),
\end{aligned}
$$

i.e. $a_{-}(n+1) \geqq a_{-}(n)$.

5.4 Definition. The smallest $\ell \in \mathbb{N}$ such that $\Gamma_{\ell}: \mathscr{B} \rightarrow \mathscr{H}^{\otimes \ell}$ has rank $k^{2}$ is called the interaction length $\ell_{0}$ of the purely generated state $\omega$. A positive operator $h \in \mathscr{A}^{\otimes \ell}$ is called an interaction exposing $\omega$, if $\ell>\ell_{0}$, and the kernel of $h$ coincides with $\mathscr{G}_{\ell}=\Gamma_{\ell}(\mathscr{B})$. The Hamiltonian of the system is then the formal expression

$$
H=\sum_{i \in \mathbb{Z}} \alpha_{i}(h)
$$

where $\alpha_{i}(h) \in \mathscr{A}_{\{i, i+1, \ldots, i+\ell-1\}}$ is the $i^{\text {th }}$ translate of $h$.

The reason for this terminology is that $\omega(h)$ represents the energy density of the Hamiltonian. By Lemma 5.1 the $\ell$-step density matrix of $\omega$ has support in $\mathscr{G}_{\ell}$, so $\omega(h)=0$, realizes the smallest possible energy density, and $\omega$ is a ground state in this sense. This is analogous to a state on a $C^{*}$-algebra being contained in the set $\{\varphi \mid \varphi(H)=0\}$ for some positive element $H$, which is usually called the face "exposed" by $H$. The "typical" interaction length of purely generated states can be obtained by a simple counting of dimensions: in the space of $k^{2} \times d^{n}$-matrices the matrices of maximal rank form an open set. Therefore, we expect $\Gamma_{n}$ to be non-singular as soon as $k^{2} \leqq d^{n}$, i.e. we expect $\ell_{0}$ to be the least integer with $\ell_{0} \geqq 2 \log k / \log d$.

It is clear that if $h$ exposes $\omega$, the $\omega$-expectations of the "finite size Hamiltonians"

$$
H_{\{n+1, \ldots, n+m\}}=\sum_{i=0}^{m-\ell} \alpha_{n+i}(h) \in \mathscr{A}_{\{n+1, \ldots, n+m\}}
$$

for $m>\ell$ also vanish. The kernel of $H_{\{1, \ldots, m\}} \in \mathscr{A}^{\otimes m}$ is clearly equal to the intersection of the kernels of the positive operators $h_{k}$. On the other hand, since $\omega\left(H_{\{1, \ldots, m\}}\right)=0$, the support $\mathscr{G}_{m}$ of the $m$-step density matrix must be contained in the kernel of $H_{\{1, \ldots, m\}}$. The following lemma asserts that these two spaces are, in fact, equal. Hence if $h \in \mathscr{A}^{\otimes \ell}$ exposes $\omega$, then so does $H_{\{1, \ldots, m\}} \in \mathscr{A}^{\otimes m}$.

5.5 Lemma. For all $m \geqq \ell>\ell_{0}$,

$$
\mathscr{G}_{m}=\bigcap_{i=0}^{m-\ell} \mathscr{H}^{\otimes i} \otimes \mathscr{G}_{\ell} \otimes \mathscr{H}^{\otimes(m-\ell-i)} .
$$

Proof. Proceeding by induction over $m$, beginning with the trivial statement for $m=\ell$, we have to show that $\mathscr{G}_{\ell+1}=\mathscr{G}_{\ell} \otimes \mathscr{H} \cap \mathscr{H} \otimes \mathscr{G}_{\ell}$, provided that $\ell>\ell_{0}$. The latter condition means that $\Gamma_{\ell-1}: \mathscr{B} \rightarrow \mathscr{G}_{\ell-1}$ is injective, i.e. that $\operatorname{Tr}\left(B v\left(\mu_{1}\right) \cdots v\left(\mu_{\ell-1}\right)\right)=0$ for all $(\ell-1)$-tuples $\left(\mu_{1}, \ldots, \mu_{\ell-1}\right)$ implies $B=0$. Now the vectors $\Phi=\sum \Phi\left(\mu_{1}, \ldots, \mu_{\ell+1}\right) \psi_{\mu_{1}} \otimes \cdots \psi_{\mu_{\ell+1}}$ in $\mathscr{G}_{\ell} \cap \mathscr{H}$ are precisely those with

$$
\Phi\left(\mu_{1}, \ldots, \mu_{\ell+1}\right)=\operatorname{Tr}\left(B\left(\mu_{\ell+1}\right) v\left(\mu_{\ell}\right)^{*} \cdots v\left(\mu_{1}\right)^{*}\right),
$$

with $B\left(\mu_{\ell+1}\right)$ an arbitrary $\mu_{\ell+1}$-dependent matrix, which is uniquely determined by $\Phi$ because $\Gamma_{\ell}$ is injective. The condition $\Phi \in \mathscr{H} \otimes \mathscr{G}_{\ell}$ can be expressed similarly 
with a $\mu_{1}$-dependent matrix $C\left(\mu_{1}\right)$. Then $\Phi \in \mathscr{G}_{\ell} \otimes \mathscr{H} \cap \mathscr{H} \otimes \mathscr{G}_{\ell}$ iff

$$
\begin{aligned}
0 & =\operatorname{Tr}\left(B\left(\mu_{\ell+1}\right) v\left(\mu_{\ell}\right)^{*} \cdots v\left(\mu_{1}\right)^{*}\right)-\operatorname{Tr}\left(C\left(\mu_{1}\right) v\left(\mu_{\ell+1}\right)^{*} v\left(\mu_{\ell}\right)^{*} \cdots v\left(\mu_{2}\right)^{*}\right) \\
& =\operatorname{Tr}\left(\left\{v\left(\mu_{1}\right)^{*} B\left(\mu_{\ell+1}\right)-C\left(\mu_{1}\right) v\left(\mu_{\ell+1}\right)^{*}\right\} v\left(\mu_{\ell}\right)^{*} \cdots v\left(\mu_{2}\right)^{*}\right) .
\end{aligned}
$$

Since this relation holds for all $(\ell-1)$-tuples $\left(\mu_{\ell}, \ldots, \mu_{2}\right)$, the expression in braces must vanish for all $\mu_{\ell+1}, \mu_{1}$. Hence using (5.3.b):

$$
B(\mu)=\sum_{v} v(v) v(v)^{*} B(\mu)=\sum_{v} v(v) C(v) v(\mu)^{*}=D v(\mu)^{*},
$$

with $D=\sum_{v} v(v) C(v)$. Hence $\Phi=\Gamma_{\ell+1}(D) \in \mathscr{G}_{\ell+1}$. The converse inclusion is trivial, since for given $D$ we can take $B(\mu)=D v(\mu)^{*}$ and $C(\mu)=v(\mu)^{*} D$.

This lemma points out an interesting feature of the structure we investigate here. Given an arbitrary subspace $\mathscr{G}_{\ell} \subset \mathscr{H}^{\otimes \ell}$ we could take the intersection in the statement of the lemma as a definition of a subspace $\mathscr{G}_{m} \subset \mathscr{H}^{\otimes m}$. Then $\mathscr{G}_{m}$ is the kernel of any positive $h \in \mathscr{A}^{\otimes \ell}$ with kernel $\mathscr{G}_{\ell}$. Obviously, the definition of "exposing interactions" depends only on these spaces, and one might try to set up a general theory of such interactions and their ground states. The problem with this is that for a generic subspace $\mathscr{G}_{\ell} \subset \mathscr{H}^{\otimes \ell}$ the intersection $\mathscr{G}_{m}$ simply becomes empty for large $m$. In fact, for $n$ generic subspaces $R_{i}$ of a vector space $R$ the inequality

$$
\operatorname{dim}\left(\bigcap_{i} R_{i}\right) \geqq \sum_{i} \operatorname{dim} R_{i}-(n-1) \operatorname{dim} R
$$

is an equality, whenever the right-hand side is non-zero. Therefore, a naive estimate of the above intersection would be

$$
\operatorname{dim} \mathscr{G}_{m} \approx(m-\ell+1) d^{m}\left(d^{-\ell} \operatorname{dim} \mathscr{G}_{\ell}-\frac{m-\ell}{m-\ell+1}\right),
$$

and this certainly becomes negative for large $m$. Thus the spaces $\mathscr{G}_{\ell}=\Gamma_{\ell}(\mathscr{B})$ are special in that these intersections stay non-empty, and it is precisely this property, which makes the existence of "exposed" states possible.

With the help of Lemma 5.5 we can now give a concise characterization of the different interactions exposing $\omega$. Since $\omega$, considered as a state on the chain $\left(\mathscr{A}^{\otimes p}\right)_{\mathbb{Z}}$ satisfies the general assumptions of this section, we may also look for interactions $h^{\prime} \in\left(\mathscr{A}^{\otimes p}\right)^{\otimes \ell^{\prime}} \equiv \mathscr{A}^{\otimes p \ell^{\prime}}$ exposing $\omega$. All these interactions are equivalent in the following strong sense.

5.6 Lemma. Let $h \in \mathscr{A}^{\otimes \ell}$ be an interaction exposing $\omega$, and let $p, \ell^{\prime} \in \mathbb{N}$ with $p \ell^{\prime}>\ell_{0}$. For $h^{\prime} \in \mathscr{A}^{\otimes p \ell^{\prime}}$, and $m \in \mathbb{N}$ let

$$
H_{\{1, \ldots, p m\}}^{\prime}=\sum_{i=0}^{m-\ell^{\prime}} \alpha_{p i}\left(h^{\prime}\right) .
$$

Suppose that $h^{\prime}$ is an interaction exposing $\omega$ considered as a state on $\left(\mathscr{A}^{\otimes p}\right)_{\mathbb{Z}}$. Then there are constants $C_{ \pm}$such that for $p m \geqq \ell+p-1$ and $m \geqq \ell^{\prime}$,

$$
C_{-} H_{\{1, \ldots, p m\}} \leqq H_{\{1, \ldots, p m\}}^{\prime} \leqq C_{+} H_{\{1, \ldots, p m\}} .
$$

Proof. Let $m_{0}$ be the smallest $m$ with $m \geqq \ell^{\prime}$ and $m \geqq(\ell+p-1) / p$. Then the 
Hamiltonians $H_{0} \equiv H_{\left\{1, \ldots, p m_{0}\right\}}$ and $H_{0}^{\prime} \equiv H_{\left\{1, \ldots, p m_{0}\right\}}^{\prime}$ are both defined in $\mathscr{A}_{\left\{1, \ldots, p m_{0}\right\}}$. Since both interactions expose $\omega$, both $H_{0}$ and $H_{0}^{\prime}$ have the same kernel, namely $\mathscr{G}_{p m_{0}}$. Hence

$$
H_{0} \leqq\left\|H_{0}\right\|\left(\mathbb{1}-G_{p m_{0}}\right) \leqq\left\|H_{0}\right\|\left(\eta^{\prime}\right)^{-1} H_{0}^{\prime},
$$

where $\eta^{\prime}$ is the smallest non-zero eigenvalue of $H_{0}^{\prime}$. Similarly, we obtain the estimate $H_{0}^{\prime} \leqq \eta^{-1}\left\|H_{0}^{\prime}\right\|$. Now for $m \geqq m_{0}$ we have

$$
\begin{aligned}
& H_{\{1, \ldots, p m\}} \leqq \sum_{i=0}^{m-m_{0}} \alpha_{p i}\left(H_{0}\right) \leqq m_{0} H_{\{1, \ldots, p m\}}, \\
& H_{\{1, \ldots, p m\}}^{\prime} \leqq \sum_{i=0}^{m-m_{0}} \alpha_{p i}\left(H_{0}^{\prime}\right) \leqq\left(m_{0}-\ell^{\prime}+1\right) H_{\{1, \ldots, p m\}}^{\prime} .
\end{aligned}
$$

This estimate follows simply by inserting the definitions of $H_{0}, H_{0}^{\prime}$, and counting how often each translate $\alpha_{i}(h), \alpha_{p i}\left(h^{\prime}\right)$ occurs in the sum in the middle. Combining the estimates we find the inequality stated in the lemma with $C_{+}=\eta^{-1}\left\|H_{0}^{\prime}\right\| m_{0} / \eta$, and $C_{-}=\eta^{\prime}\left\|H_{0}\right\|^{-1}\left(m_{0}-\ell+1\right)^{-1}$.

5.7 Theorem. Let $h \in \mathscr{A}^{\otimes \ell}$ be an interaction exposing $\omega$. Then $\omega$ is the unique state on $\mathscr{A}_{\mathbb{Z}}$ such that

$$
\omega\left(\alpha_{i}(h)\right)=0
$$

for all $i \in \mathbb{Z}$.

Proof. Let $\tilde{\omega}$ be a state with $\tilde{\omega} \alpha_{i}(h) \equiv 0$. Then for all $i \in \mathbb{Z}$ and $n \geqq \ell$ the density matrix $W_{\{i+1, \ldots, i+n\}}$ of $\tilde{\omega} \mid \mathscr{A}_{\{i+1, \ldots, i+n\}}$ is supported by the subspace $\bigcap_{s=0} \mathscr{H}^{\otimes s} \otimes \mathscr{G}_{\ell} \otimes \mathscr{H}^{\otimes(n-s-\ell)}$. Hence by Lemma $5.5 W_{\{i+1, \ldots, i+n\}}$ is supported by $\mathscr{G}_{n}$ for all $i, n$. Thus we have a representation $W_{\{i+1, \ldots, i+n\}}=\sum_{s}\left|\Gamma_{n}\left(B_{s}\right)\right\rangle\left\langle\Gamma_{n}\left(B_{s}\right)\right|$ with $B_{s} \in \mathscr{B}$, and $\sum_{s}\left\|\Gamma_{n}\left(B_{s}\right)\right\|^{2}=1$. For $A \in \mathscr{A}_{\{j+1, \ldots, j+m\}}$ we apply this representation and Lemma 5.2(2) with sufficiently small $i$ and sufficiently large $n$ to obtain

$$
\begin{aligned}
|\tilde{\omega}(A)-\omega(A)| & =\left|\sum_{s}\left\{\left\langle\Gamma_{n}\left(B_{s}\right), A \Gamma_{n}\left(B_{s}\right)\right\rangle-\omega(A)\left\langle\Gamma_{n}\left(B_{s}\right), \Gamma_{n}\left(B_{s}\right)\right\rangle\right\}\right| \\
& \leqq(a(j-i)+a(n+i-j-m))\|A\| \sum_{s}\left\|B_{s}\right\|_{\rho}^{2} \\
& \leqq(a(j-i)+a(n+i-j-m)) a_{-}(n)^{-1}\|A\| .
\end{aligned}
$$

For small $i$ and large $n$ the bracket can be made arbitrarily small.

5.8 Definition. $A$ VBS interaction is an interaction $h$ of finite range $\ell, h \in \mathscr{A}^{\otimes \ell}$, with the following property: there exists a $C^{*}$-finitely correlated state $\omega$ such that

(1) $h \geqq 0$ and $\omega(h)=0$,

(2) for all $n \geqq \ell$ let $H_{\{1, \ldots, n\}}=\sum_{i=1} \alpha_{i}(h)$ be the local Hamiltonian corresponding to

the interaction $h$ and let $\eta$ be any ground state of $H_{\{1, \ldots, n\}}$, i.e a state of $\mathscr{A}_{\{1, \ldots, n\}}$ such that $\eta\left(H_{\{1, \ldots, n\}}\right)=0$; then there exists a constant $C>0$ such that $\eta \leqq\left. C \omega\right|_{\mathscr{A}\{1, \ldots, n\}}$. 
At this point we want to remark that for a given purely generated and exponentially clustering $C^{*}$-finitely correlated state $\omega$, Theorem 5.7 guarantees the existence of a VBS interaction $h$ such that $\omega$ is the unique zero energy ground state for the corresponding Hamiltonian. If the interaction length of $\omega$ is $\ell_{0}$, there always exists an interaction $h$ of range $\ell_{0}+1$ exposing $\omega$. Such an interaction is a good VBS interaction in the sense of Definition 5.8. In some cases the intersection property of Lemma 5.5 already holds for $\ell=\ell_{0}$. In such cases one can replace the Hamiltonian of Theorem 5.7 by an equivalent VBS interaction of range $\ell_{0}$. The family of examples introduced in Example 1 turns out to have this property as will become clear in Example 7.

Moreover note that the fact that for a given interaction all ergodic infinite volume ground states are $C^{*}$-finitely correlated, does not imply that it is a VBS interaction. The simplest example of this situation is given by the spin $1 / 2$ Heisenberg ferromagnet and we will consider this in more detail in Example 5.

In view of Corollary 3.4 and the definition of VBS models of above we must conclude that only certain types of symmetry breaking in the ground state can occur. Indeed a $C^{*}$-finitely correlated state can be decomposed into at most a finite number of ergodic (or periodic) components. In particular one cannot have genuine breaking of a continuous local symmetry. Breaking of a continuous symmetry can occur but in such a case also the translation symmetry is fully broken (no periodicity is left) and residual entropy is generated. A detailed analysis of such an example is given in [31]. Examples of breaking of translation symmetry into periodic states are given in Examples 4 and 6 (the Majumdar-Ghosh model).

Example 5. Any pure translation invariant product state of a spin $1 / 2$ chain is a ground state for the Heisenberg ferromagnet. For this model to be a VBS model there should exist a $C^{*}$-finitely correlated ground state $\omega$, which locally dominates each of these ground states. We will show that this is not the case. Let us first recall some well-known facts about the ground states of this model [40]. The local Hamiltonians $H_{\{1, \ldots, n\}}$ are given as:

$$
H_{\{1, \ldots, n\}}=\sum_{i=1}^{n-1} P_{i, i+1}^{0},
$$

where $P^{0}$ is the orthogonal projection on the singlet state in $\mathbb{C}^{2} \otimes \mathbb{C}^{2}$ which can equivalently be written as $P^{0}=\frac{1}{2}(\mathbb{1}-U)$. Here $U$ is the unitary operator which flips the factors in $\mathbb{C}^{2} \otimes \mathbb{C}^{2}$. It is easy to check that there is a state $\omega$ such that $\omega\left(P_{i, i+1}^{0}\right)=0$ and these equations characterize the ground states of the ferromagnet. In order to get the general solution of this equation observe that $\omega\left(P_{i, i+1}^{0}\right)=0$ implies that for any local observable $A$ and for any $i \in \mathbb{Z}: \omega(A)=\omega\left(U_{i, i+1} A\right)=$ $\omega\left(U_{i, i+1} A U_{i, i+1}\right)$. Therefore any ground state $\omega$ will be fully symmetric, i.e. $\omega$ is invariant under arbitrary finite permutations of sites. It is then a standard result by St $\phi$ rmer [61] that such an $\omega$ can be uniquely written as:

$$
\omega=\int_{\mathscr{T}_{1}^{+}} \mu(d \sigma) \bigotimes_{i \in \mathbb{Z}} \sigma_{i}
$$

In this formula $\mathscr{T}_{1}^{+}$denotes the $2 \times 2$ density matrices, $\mu(d \sigma)$ a probability measure on $\mathscr{T}_{1}^{+}$and $\underset{i \in \mathbb{Z}}{\bigotimes} \sigma_{i}$ is the product state on the spin chain determined by the density matrix $\sigma$. As $\sigma \otimes \sigma\left(P^{0}\right)=\frac{1}{2}\left((\operatorname{Tr} \sigma)^{2}-\operatorname{Tr} \sigma^{2}\right)$, it follows that $\sigma$ has to be pure in order 
that $\bigotimes_{i \in \mathbf{Z}} \sigma_{i}$ be a ground state. We will now contradict the assumption that the Heisenberg ferromagnet is a VBS model. More precisely we will show that there is no $C^{*}$-finitely correlated ground state $\omega_{0}$ that locally dominates all the other ground states. Indeed, suppose that $\omega_{0}$ is such a $C^{*}$-finitely correlated state, then let $\mu_{0}(d \sigma)$ be the probability measure that defines $\omega_{0}$ as in formula (5.12) and $K_{0}$ the support of $\mu_{0}$. The minimal representation of $\omega_{0}$ is then given as follows:

(1) $\mathscr{B}=\mathscr{C}\left(K_{0}\right)$, the continuous, complex-valued functions on $K_{0}$.

(2) $\mathbb{E}(A \otimes f)(\sigma)=\sigma(A) f(\sigma), \mathscr{A} \in \mathscr{M}_{2}$ and $f \in \mathscr{C}\left(K_{0}\right)$.

As $\mathscr{B}$ is abelian and $\sigma$ is a density matrix $\mathbb{E}$ is completely positive.

(3) To complete the triple $(\mathbb{E}, \rho, e)$ we take $e=1$ and $\rho(f)=\int \mu_{0}(d \sigma) f(\sigma)$. The conditions (2.2) are now easily verified:

$$
\begin{aligned}
\mathbb{E}(\mathbb{1} \otimes 1)(\sigma) & =\sigma(\mathbb{1}) 1=1, \\
\rho(\mathbb{E}(\mathbb{1} \otimes f)) & =\int \mu_{0}(d \sigma) \sigma(\mathbb{1}) f(\sigma)=\rho(f) .
\end{aligned}
$$

With these definitions it is straightforward to verify that we recover the state $\omega_{0}$ using the defining formula (2.1). Finally the minimality of the representation follows from the Weierstraß Theorem. As $\omega_{0}$ is $C^{*}$-finitely correlated $\mathscr{B}$ has to be finite-dimensional, i.e. $K_{0}$ is a finite set. As $\omega_{0}$ is a ground state the elements of $K_{0}$ have to be pure states. It now follows that the restriction of $\omega_{0}$ to any finite volume is a convex combination of at most $\#\left(K_{0}\right)$ vector states. As the degeneracy of the ground state of the Hamiltonian $H_{\{1, \ldots, n\}}$ is $n+1$ clearly $\omega_{0}$ cannot dominate all these ground states.

Example 6. The Majumdar-Ghosh Model [53,54]. This model lives on a spin 1/2 chain and the formal Hamiltonian $H$ is given by:

$$
H_{\mathrm{MG}}=\sum_{i \in \mathbb{Z}} P_{i, i+1, i+2}^{3 / 2},
$$

where $P^{3 / 2}$ is the orthogonal projection onto the spin $3 / 2$ subspace of $\mathbb{C}^{2} \otimes \mathbb{C}^{2} \otimes \mathbb{C}^{2}$ which can be expressed in terms of the generators of $S U(2)$ as:

$$
P_{1,2,3}^{3 / 2}=\frac{2}{3}\left(\mathbf{S}_{1} \cdot \mathbf{S}_{2}+\mathbf{S}_{2} \cdot \mathbf{S}_{3}+\mathbf{S}_{1} \cdot \mathbf{S}_{3}\right)+\frac{1}{2} \mathbb{1} \text {. }
$$

We now specify the triple $(\mathbb{E}, \rho, \mathbb{1})$ that will determine the unique translation invariant ground state $\omega_{\mathrm{MG}}$ of this model [5]. As auxiliary algebra $\mathscr{B}$ we take $\mathscr{M}_{2} \oplus \mathbb{C}$. Denote by $\varphi$ the singlet state on $\mathscr{M}_{2} \otimes \mathscr{M}_{2}$. We now define the completely positive map $\mathbb{E}$ by

$$
\begin{aligned}
\mathbb{E}: \mathscr{M}_{2} \otimes\left(\mathscr{M}_{2} \oplus \mathbb{C}\right) & \rightarrow \mathscr{M}_{2} \oplus \mathbb{C}, \\
A \otimes\left(B_{1} \oplus B_{2}\right) & \rightarrow B_{2} A \otimes \varphi\left(A \otimes B_{1}\right) .
\end{aligned}
$$

The state $\rho$ on $\mathscr{M}_{2} \oplus \mathbb{C}$ has to be taken as

$$
\rho\left(B_{1} \oplus B_{2}\right)=\frac{1}{2} B_{2}+\frac{1}{2} \varphi\left(\mathbb{1} \otimes B_{1}\right) .
$$

Again we have to check the relations (2.2). It is obvious that $\mathbb{E}$ is unity preserving. We still have to verify that for all $B \in \mathscr{M}_{2} \oplus \mathbb{C}, \rho(\mathbb{E}(\mathbb{1} \otimes B))=\rho(B)$. Indeed

$$
\begin{aligned}
\rho\left(\mathbb{E}\left(\mathbb{1} \otimes\left(B_{1} \oplus B_{2}\right)\right)\right. & =\rho\left(B_{2} \mathbb{1} \oplus \varphi\left(\mathbb{1} \otimes B_{1}\right)\right)=\frac{1}{2} \varphi\left(\mathbb{1} \otimes B_{1}\right)+\frac{1}{2} \varphi\left(B_{2} \mathbb{1} \otimes \mathbb{1}\right) \\
& =\frac{1}{2} \varphi\left(\mathbb{1} \otimes B_{1}\right)+\frac{1}{2} B_{2}=\rho(B) .
\end{aligned}
$$

To see that the $C^{*}$-finitely correlated state $\omega_{M G}$ is indeed a ground state of the 
Hamiltonian $H_{\mathrm{MG}}$ one checks that the state $\omega_{\mathrm{MG}}$ is rotation invariant and belongs to spin $\leqq 1 / 2$ on any set of an odd number of consecutive sites. Therefore it must be a ground state. For a more complete discussion see e.g. [5]. The diagonalization of $\hat{\mathbb{E}}$ is given by:

$$
\hat{\mathbb{E}}(\mathbb{1} \oplus 1)=\mathbb{1} \oplus 1, \quad \hat{\mathbb{E}}(\mathbb{1} \oplus-1)=-(\mathbb{1} \oplus-1), \quad \hat{\mathbb{E}}\left(S^{\alpha} \oplus 0\right)=0, \quad \alpha=x, y, z .
$$

So we find two eigenvalues with modulus 1, and by Proposition $3.3 \omega_{\mathrm{MG}}$ can be decomposed into two ergodic 2-periodic states. The two components are pure and are the ground states of the Majumdar-Ghosh model as they appear in the original papers.

Example 7. We show that for our family of models the interaction length $\ell_{0}=2$ and that the intersection property of Lemma 5.5 holds for $\ell=2$.

(1) $\cos \theta \sin \theta \neq 0$. The range $\mathscr{G}_{2}$ of $\Gamma_{2}$ is spanned by the vectors:

$$
\begin{gathered}
\sqrt{\frac{1}{2}}(|1,0\rangle-|0,1\rangle), \quad \sqrt{\frac{1}{2}}(|1,-1\rangle-|-1,1\rangle), \quad \sqrt{\frac{1}{2}}(|0,-1\rangle-|-1,0\rangle), \\
\frac{1}{\sqrt{2 \cos ^{4} \theta+4 \sin ^{4} \theta}}\left(\cos ^{2} \theta|1,-1\rangle-2 \sin ^{2} \theta|0,0\rangle+\cos ^{2} \theta|-1,1\rangle\right) .
\end{gathered}
$$

It follows that the interaction length $\ell_{0}$ of $\omega_{\theta}$ equals 2 . As mentioned above the intersection property of Lemma 5.5 holds for $\ell=2$, i.e. $\mathscr{G}_{3}=\mathscr{G}_{2} \otimes \mathbb{C}^{3} \cap \mathbb{C}^{3} \otimes \mathscr{G}_{2}$. By Lemma $5.34 \geqq \operatorname{dim} \mathscr{G}_{3} \geqq \operatorname{dim} \mathscr{G}_{2}=4$ and, as $\mathscr{G}_{3} \subset \mathscr{G}_{2} \otimes \mathbb{C}^{3} \cap \mathbb{C}^{3} \otimes \mathscr{G}_{2}$, it is sufficient to show that $\operatorname{dim}\left(\mathscr{G}_{2} \otimes \mathbb{C}^{3} \cap \mathbb{C}^{3} \otimes \mathscr{G}_{2}\right) \leqq 4$. This is straightforward to check using the obvious symmetries of $\mathscr{G}_{2}$ : rotations about the $z$-axis, spin flip and space reflection.

It is now clear that any positive nearest neighbor interaction $h_{\theta}$ with $\operatorname{ker} h_{\theta}=\mathscr{G}_{2}$, will lead to a VBS model having $\omega_{\theta}$ as its unique ground state. In particular we can take the projection operator on the orthogonal complement of $\mathscr{G}_{2}$. This operator can be expressed in terms of the spin operators. Thus we obtain the Hamiltonians given in (1.2):

$$
\begin{aligned}
H_{\theta}= & \sum_{i}\left\{\frac{1}{2} \mathbf{S}_{i} \cdot \mathbf{S}_{i+1}+\left(\frac{1}{6}+\frac{1}{3} \eta\right)\left(\mathbf{S}_{i} \cdot \mathbf{S}_{i+1}\right)^{2}+\left(\frac{1}{3}-\frac{4}{3} \eta\right)\right. \\
& \left.+\eta\left(S_{i}^{z}\right)^{2}-\frac{1-t}{1+t} \eta\left(S_{i}^{z} S_{i+1}^{z}\right)^{2}+\frac{1-t}{1+t} \frac{\eta}{2}\left\{S_{i}^{z} S_{i+1}^{z}, \mathbf{S}_{i} \cdot \mathbf{S}_{i+1}\right\}\right\},
\end{aligned}
$$

where $t=\sin ^{2} \theta$ and $\eta=\left(4 \sin ^{4} \theta-\cos ^{4} \theta\right) /\left(2\left(\cos ^{4} \theta+2 \sin ^{4} \theta\right)\right)$.

(2) $\theta=0$. The spaces $\mathscr{G}_{n}$ are two-dimensional and spanned by the vectors

$$
|1\rangle \otimes|-1\rangle \otimes \cdots\left|(-1)^{n+1}\right\rangle, \quad|-1\rangle \otimes|1\rangle \otimes \cdots\left|(-1)^{n}\right\rangle .
$$

These are the ground states of the following Hamiltonian:

$$
H_{\{1, \ldots, n\}}=\sum_{i=1}^{n-1}\left\{S_{i}^{z} S_{i+1}^{z}+1\right\} .
$$

Clearly this Hamiltonian is a VBS model in the sense of Definition 5.8. Although it is not the limit for $\theta \rightarrow 0$ of $H_{\theta}$, we know by Lemma 5.6 that both models are equivalent. 
(3) $\theta=\pi / 2$. The spaces $\mathscr{G}_{n}$ are now one-dimensional and determined by the vectors $|0\rangle \otimes|0\rangle \otimes \cdots|0\rangle$. There exists in this case a completely trivial VBS interaction:

$$
H_{\{1, \ldots, n\}}=\sum_{i=1}^{n}\left(S_{i}^{z}\right)^{2} .
$$

Again it is equivalent with but not equal to $H_{\pi / 2}$.

We close this section with a collection of properties of $\omega$, which are immediate consequences of the foregoing.

5.9 Proposition. $\omega$ is a pure state on $\mathscr{A}_{\mathbb{Z}}$ with zero entropy density. The non-zero eigenvalues of the density matrix of $\omega \mid \mathscr{A}^{\otimes n}$ converge to the numbers $\left\{\rho_{\alpha} \cdot \rho_{\beta}\right\}_{\alpha, \beta=1}^{k}$, where $\left\{\rho_{\alpha}\right\}_{\alpha=1}^{k}$ are the eigenvalues of $\rho$. The limiting absolute entropy of $\omega$ is twice the entropy of $\rho$.

Proof. Any convex component $\tilde{\omega} \leqq \lambda \omega$ of $\omega$ satisfies the condition of the theorem, and is hence equal to $\omega$. Since the $n$-step density matrix is supported by $\mathscr{G}_{n}$, which has dimension $k^{2}$ for large $n$, its entropy is bounded by $2 \ln (k)$, so the entropy per site vanishes as $n \rightarrow \infty$. By Lemma 5.1.(1) the $n$-step density matrix is $\Gamma_{n} W_{\infty} \Gamma_{n}{ }^{*}$, and since $\Gamma_{n}$ becomes an isometry in the limit, we merely have to compute the eigenvalues of $W_{\infty}(B)=\rho B \rho$. The eigenvectors of $W_{\infty}$ are $B=\left|\chi_{\alpha}\right\rangle\left\langle\chi_{\beta}\right|$, where $\left\{\chi_{\alpha}\right\}$ is an eigenbasis of $\rho$, so the eigenvalues are $\rho_{\alpha} \rho_{\beta}$. The limiting entropy of $\omega \mid \mathscr{A}^{\otimes n}$ is the entropy of $W_{\infty} \cong \rho \otimes \rho$.

One can show that a $C^{*}$-finitely correlated state with vanishing entropy density is necessarily purely generated [33]. One can also find VBS interactions with a (non-unique) $C^{*}$-finitely correlated ground state having positive entropy density [31].

\section{The Ground State Energy Gap}

There are two natural ways of looking at the infinite sum in the formal Hamiltonian $H=\sum_{n \in \mathbb{Z}} \alpha_{n}(h)$. The first is to discuss only energy densities, i.e. the expectations of the individual terms in this sum. For example, in the last section we considered states, in which each term had zero expectation so that we never had to consider the convergence of the sum. Another natural approach is to consider the Hamiltonian not as an observable, but as the generator of the dynamical automorphism group $t \mapsto \tau_{t} \in$ Aut $\mathscr{A}_{\mathbb{Z}}$. More precisely, the generator of this group is the closure of $X \mapsto i[H, X]$, defined on strictly local operators $X$. For such $X$ only a finite number of terms in the Hamiltonian contributes to the commutator. The notion of "ground state" corresponding to the latter view of the Hamiltonian is the inequality

$$
\omega\left(X^{*}[H, X]\right) \geqq 0 \text { for all local } X \in \mathscr{A}_{\mathbb{Z}} .
$$

This is equivalent to the positivity of the Hamiltonian $H_{\omega}$, which is defined in the GNS-representation $\left(\pi_{\omega}, \mathscr{H}_{\omega}, \Omega_{\omega}\right)$ of the state $\omega$ by

$$
\pi_{\omega}\left(\tau_{t}(X)\right) \Omega_{\omega}=e^{i t H_{\omega}} \pi_{\omega}(X) \Omega_{\omega} .
$$


When $h \in \mathscr{A}^{\otimes \ell}$ is an interaction exposing $\omega$ in the sense of Definition 5.4, we have for $X \in \mathscr{A}_{\{n, \ldots, n+m\}}$ :

$$
\omega\left(X^{*}[H, X]\right)=\omega\left(X^{*}\left[H_{\{n-\ell, \ldots, n+m+\ell\}}, X\right]\right)=\omega\left(X^{*} H_{\{n-\ell, \ldots, n+m+\ell\}} X\right),
$$

since for all $n \in \mathbb{Z}$ we have $\omega\left(X^{*} X \alpha_{n}(h)\right)=0$. Therefore, the positivity of $H_{\{n-\ell, \ldots, n+m+\ell\}}$ implies that the ground states considered in the previous section are also ground states in the sense of inequality (6.1). It is known [16] that, conversely, inequality (6.1) implies the minimum energy density property for translation invariant states.

In this section we want to investigate the existence of gaps above the ground state. Again there will be two notions of "gap." The first is to replace the positivity of $H_{\{n, \ldots, n+m\}}$ by the stronger requirement that the first non-zero eigenvalue of this operator is bounded below by a constant $\gamma>0$, independently of $n$ and $m \geqq \ell$. The second notion is to postulate that $H_{\omega}$ has a spectral gap, i.e. that the eigenvalue zero is isolated from the remainder of the spectrum by an interval of length $\gamma$. This is equivalent to the inequality

$$
\omega\left(X^{*}[H, X]\right) \geqq \gamma\left\{\omega\left(X^{*} X\right)-|\omega(X)|^{2}\right\}
$$

for all local $X \in \mathscr{A}_{\mathbb{Z}}$. Again we can use Eq. (6.3) to simplify this expression, so that only the finite volume Hamiltonians $H_{\{n, \ldots, n+m\}}$ appear. The first notion of gap is meaningless as such. Indeed, even if there is a unique global ground state, boundary terms in local Hamiltonians that still lead to the same global dynamics may produce degeneracies or perturb the local gaps [37].

The following lemma shows that for the states under consideration a gap in the first sense implies the inequality (6.4).

6.1 Lemma. Let $h \in \mathscr{A}^{\otimes \ell}$ be an interaction exposing the $C^{*}$-finitely correlated state $\omega$. Suppose that for infinitely many $m \in \mathbb{N}$ the first non-zero eigenvalue of $H_{\{1, \ldots, m\}}$ is larger than $\gamma>0$. Then inequality (6.4) holds.

Proof. Let $X \in \mathscr{A}_{\mathbb{Z}}$ be local. Then by translation invariance of $H$ and $\omega$ we may assume $X \in \mathscr{A}_{\{1, \ldots, m\}}$. Since neither side of inequality (6.4) changes, if we replace $X$ by $X-\omega(X) \mathbb{1}$, we may also assume that $\omega(X)=0$. Consider for each $L$ the vector

$$
\Psi_{L}=\left(\mathbb{1}_{L} \otimes X \otimes \mathbb{1}_{L} \Gamma_{L+m+L}(\mathbb{1})\right) \in \mathscr{H}^{\otimes L} \otimes \mathscr{H}^{\otimes m} \otimes \mathscr{H}^{\otimes L} .
$$

We abbreviate by $H^{L}$ the Hamiltonian $H_{\{1-L, \ldots, m+L\}}$ acting in this space, and its ground state projection by $G^{L}$. By assumption, $H^{L} \geqq \gamma\left(\mathbb{1}-G^{L}\right)$ for infinitely many $L$. Then by Lemma 5.2.(2) we have $\left\|\Psi_{L}\right\|^{2}=\omega\left(X^{*} X\right)+\mathbf{O}(a(L))$, and for an arbitrary vector $\Gamma_{2 L+m}(B)$ in the range of $G^{L}$ we have

$$
\begin{aligned}
\left\langle\Gamma_{2 L+m}(B), \Psi_{L}\right\rangle & =\omega(X)\langle B, \mathbb{1}\rangle_{\rho}+\|B\|_{\rho} \cdot \mathbf{O}(a(L)) \\
& =\left\|\Gamma_{2 L+m}(B)\right\| \cdot \mathbf{O}(a(L)) .
\end{aligned}
$$

Hence $\left\|G^{L} \Psi_{L}\right\|=\mathbf{O}(a(L))$. Using Lemma 5.2.(2) once more we find

$$
\begin{aligned}
\omega\left(X^{*}[H, X]\right) & =\omega\left(X^{*} H_{\{1-\ell, \ldots, m+\ell\}} X\right)=\left\langle\Psi_{L}, H_{\{1-\ell, \ldots, m+\ell\}} \Psi_{L}\right\rangle-\mathbf{O}(a(L-\ell)) \\
& =\left\langle\Psi_{L}, H^{L} \Psi_{L}\right\rangle-\mathbf{O}(a(L)) \\
& \geqq \gamma\left\langle\Psi_{L},\left(\mathbb{1}-G^{L}\right) \Psi_{L}\right\rangle-\mathbf{O}(a(L))=\gamma \omega\left(X^{*} X\right)-\mathbf{O}(a(L)) .
\end{aligned}
$$

The result follows by letting $L \rightarrow \infty$. 
It is clear from Lemma 5.6 and Eq. (6.3) that if one interaction exposing $\omega$ has a non-zero gap, then all other such interactions will have the same property. The special interaction, for which we shall prove this property in Theorem 6.4 will be of the form $\left(\mathbb{1}-G_{2 p}\right)$ for some $p$. The following lemma establishes the basic estimate for ground state projections needed in the proof of 6.4 .

6.2 Lemma. For all $\ell, m, r \in \mathbb{N}$, with $m \geqq \ell_{0}$, and $a(m), a_{-}(m)$ as in Lemma 5.2:

$$
\left\|\left(G_{\ell+m} \otimes \mathbb{1}_{r}\right)\left(\mathbb{1}_{\ell} \otimes G_{m+r}\right)-G_{\ell+m+r}\right\| \leqq a(m) \frac{1+a(m)}{a_{-}(m)}
$$

Proof. Since $G_{\ell+m+r} \leqq\left(G_{\ell+m} \otimes \mathbb{1}_{r}\right)$, we can write $\left(G_{\ell+m} \otimes \mathbb{1}_{r}\right)\left(\mathbb{1}_{\ell} \otimes G_{m+r}\right)-$ $G_{\ell+m+r}=\left(G_{\ell+m} \otimes \mathbb{1}_{r}-G_{\ell+m+r}\right)\left(\mathbb{1}_{\ell} \otimes G_{m+r}-G_{\ell+m+r}\right)$. Therefore, we have to prove the following statement: for any vectors $\Phi \in \mathscr{G}_{\ell+m} \otimes \mathscr{H}^{\otimes r}$ and $\Psi \in \mathscr{H}^{\otimes l} \otimes$ $\mathscr{G}_{m+r}$ such that $\Phi, \Psi \perp \mathscr{G}_{\ell+m+r}$, we have $|\langle\Phi, \Psi\rangle| \leqq R H S \cdot\|\Phi\| \cdot\|\Psi\|$. We shall write all vectors in components with respect to a basis $\left\{\psi_{\mu}\right\}_{\mu=1}^{\mu} \subset \mathscr{H}$, grouping the $(\ell+m+r)$-tuple of indices into three tuples $\mu^{\ell}, \mu^{m}, \mu^{r}$ of lengths $\ell, m, r$, respectively. We use the abbreviation $v\left(\mu^{m}\right)=v\left(\mu_{\ell+1}\right) v\left(\mu_{\ell+2}\right) \cdots v\left(\mu_{\ell+m}\right)$, and similar ones for $v\left(\mu^{\ell}\right)$ and $v\left(\mu^{r}\right)$. Then by definition of $\Gamma_{n}$ we can write the components of $\Phi$ and $\Psi$ in the form

$$
\begin{aligned}
& \Phi\left(\mu^{\ell}, \mu^{m}, \mu^{r}\right)=\operatorname{Tr}\left(\hat{\Phi}\left(\mu^{r}\right) v\left(\mu^{m}\right)^{*} v\left(\mu^{\ell}\right)^{*}\right), \\
& \Psi\left(\mu^{\ell}, \mu^{m}, \mu^{r}\right)=\operatorname{Tr}\left(\hat{\Psi}\left(\mu^{\ell}\right) v\left(\mu^{r}\right)^{*} v\left(\mu^{m}\right)^{*}\right),
\end{aligned}
$$

where $\hat{\boldsymbol{\Phi}}\left(\mu^{r}\right), \hat{\boldsymbol{\Psi}}\left(\mu^{\ell}\right) \in \mathscr{M}_{k}$ for each tuple $\mu^{r}$ or $\mu^{\ell}$.

We show first an estimate of $\langle\Phi, \Psi\rangle$, which does not use the orthogonality of these vectors to $\mathscr{G}_{\ell+m+r}$, namely

$$
\left|\langle\Phi, \Psi\rangle-\left\langle\Delta^{\Phi}, \Delta_{\Psi}\right\rangle_{\rho}\right| \leqq \frac{a(m)}{a_{-}(m)}\|\Phi\| \cdot\|\Psi\|,
$$

where

$$
\Delta^{\Phi}=\sum_{\mu^{r}} \hat{\Phi}\left(\mu^{r}\right) \rho v\left(\mu^{r}\right) \rho^{-1} \quad \text { and } \quad \Delta_{\Psi}=\sum_{\mu^{\prime}} v\left(\mu^{\ell}\right) \hat{\Psi}\left(\mu^{\ell}\right)
$$

Upon noting that

$$
\langle\Phi, \Psi\rangle=\sum_{\mu^{\prime}, \mu^{r}}\left\langle\Gamma_{m}\left(v\left(\mu^{\ell}\right)^{*} \hat{\Phi}\left(\mu^{r}\right)\right), \quad \Gamma_{m}\left(\hat{\Psi}\left(\mu^{\ell}\right) v\left(\mu^{r}\right)^{*}\right)\right\rangle,
$$

we can use Lemma 5.2 to write this as

$$
\begin{gathered}
\sum_{\mu^{\prime}, \mu^{r}}\left\langle v\left(\mu^{\ell}\right)^{*} \hat{\Phi}\left(\mu^{r}\right), \quad \hat{\Psi}\left(\mu^{\ell}\right) v\left(\mu^{r}\right)^{*}\right\rangle_{\rho^{\prime}}=\sum_{\mu^{\prime}, \mu^{r}} \operatorname{Tr}\left(\rho \hat{\Phi}\left(\mu^{r}\right)^{*} v\left(\mu^{\ell}\right) \hat{\Psi}\left(\mu^{\ell}\right) v\left(\mu^{r}\right)^{*}\right) \\
=\sum_{\mu^{\ell}, \mu^{r}} \operatorname{Tr}\left(\rho\left\{\hat{\Phi}\left(\mu^{r}\right) \rho v\left(\mu^{r}\right) \rho^{-1}\right\}^{*}\left\{v\left(\mu^{\ell}\right) \hat{\Psi}\left(\mu^{\ell}\right)\right\}\right)=\left\langle\Delta^{\Phi}, \Delta_{\Psi}\right\rangle_{\rho},
\end{gathered}
$$

and a remainder, which is bounded by

$$
a(m) \sum_{\mu^{\prime}, \mu^{r}}\left\|v\left(\mu^{\ell}\right)^{*} \hat{\Phi}\left(\mu^{r}\right)\right\|_{\rho} \cdot\left\|\hat{\boldsymbol{\Psi}}\left(\mu^{\ell}\right) v\left(\mu^{r}\right)^{*}\right\|_{\rho}
$$

This sum is estimated with the Cauchy-Schwarz inequality, using

$$
\sum_{\mu^{\ell}, \mu^{r}}\left\|v\left(\mu^{\ell}\right)^{*} \hat{\Phi}\left(\mu^{r}\right)\right\|_{\rho}^{2}=\sum_{\mu^{\ell}, \mu^{r}} \operatorname{Tr}\left(\rho \hat{\Phi}\left(\mu^{r}\right)^{*} v\left(\mu^{\ell}\right) v\left(\mu^{\ell}\right)^{*} \hat{\Phi}\left(\mu^{r}\right)\right)
$$




$$
\begin{aligned}
& =\sum_{\mu^{r}} \operatorname{Tr}\left(\rho \hat{\Phi}\left(\mu^{r}\right)^{*} \hat{\Phi}\left(\mu^{r}\right)\right) \leqq a_{-}(\ell+m)^{-1} \sum_{\mu^{r}}\left\|\Gamma_{\ell+m}\left(\hat{\Phi}\left(\mu^{r}\right)\right)\right\|^{2} \\
& =a_{-}(\ell+m)^{-1}\|\Phi\|^{2} \leqq a_{-}(m)^{-1}\|\Phi\|^{2}
\end{aligned}
$$

and a similar computation for $\Psi$, using $\sum_{\mu^{r}} v\left(\mu^{r}\right)^{*} \rho v\left(\mu^{r}\right)=\rho$. This yields the error estimate given in (6.5).

Equation (6.5) takes a particularly simple form if $\Phi$ (respectively $\Psi$ ) is in the subspace $\mathscr{G}_{\ell+m+r}$, say equal to $\chi=\Gamma_{\ell+m+r}(\hat{\chi})$ with $\hat{\chi} \in \mathscr{M}_{k}$. This condition is equivalent to the special form $\hat{\Phi}\left(\mu^{r}\right)=\hat{\chi} v\left(\mu^{r}\right)^{*}$ (respectively $\left.\Psi\left(\mu^{\prime}\right)=v\left(\mu^{\prime}\right)^{*} \hat{\chi}\right)$. We then have $\Delta^{\Phi}=\hat{\chi}$ (respectively $\left.\Delta_{\psi}=\hat{\chi}\right)$, and that the sum $\sum_{\mu^{\ell}, \mu^{r}}\left\|v\left(\mu^{\ell}\right)^{*} \hat{\Phi}\left(\mu^{r}\right)\right\|_{\rho}^{2}$ appearing in the error estimate of (6.5) is equal to $\|\hat{\chi}\|_{\rho}^{2}$.

If $\Psi \perp \mathscr{G}_{\ell+m+r}$, we then find that for all $\hat{\chi} \in \mathscr{M}_{k}$, $\left|\left\langle\hat{\chi}, \Delta_{\Psi}\right\rangle_{\rho}\right| \leqq a(m) a_{-}(m)^{-1 / 2}\|\hat{\chi}\|_{\rho} \cdot\|\Psi\|$.

In other words, $\|\Delta \psi\|_{\rho} \leqq a(m) a_{-}(m)^{-1 / 2}\|\Psi\|$. Together with the analogous estimate for $\left\|\Delta^{\Phi}\right\|$ and (6.5) we finally obtain

$$
\begin{aligned}
|\langle\Phi, \Psi\rangle| & \leqq\left|\left\langle\Delta^{\Phi}, \Delta_{\Psi}\right\rangle\right|+a(m) / a_{-}(m)\|\Phi\|\|\Psi\| \\
& \leqq\left(a(m)^{2} / a_{-}(m)+a(m) / a_{-}(m)\right)\|\Phi\|\|\Psi\| .
\end{aligned}
$$

In the following Lemma $E \wedge F$ and $E \vee F$ denote the largest lower bound and least upper bound in the lattice of projections, respectively.

6.3 Lemma. Let $E$ and $F$ be orthogonal projections on a finite-dimensional Hilbert space $\mathscr{H}$ then:

(1) $\|E F-E \wedge F\|=\|(\mathbb{1}-E)(\mathbb{1}-F)-(\mathbb{1}-E) \wedge(\mathbb{1}-F)\|$,

(2) $E F+F E \geqq-\|E F-E \wedge F\|(E+F)$.

Proof. Both $E \vee F$ and $E \wedge F$ reduce all the operators that appear in the statement of the lemma. The inequality (2) is trivially satisfied on $(E \vee F)^{\perp}$ and on $E \wedge F$ and both $E F-E \wedge F$ and the corresponding expression for the orthogonal complements vanish on $(E \vee F)^{\perp} \mathscr{H}$ and $(E \wedge F) \mathscr{H}$. We can therefore as well suppose that $E \vee F=\mathbb{1}$ and $E \wedge F=0$.

(1) Since $\mathscr{H}$ is finite-dimensional, we can find unit vectors $\Phi, \Psi \in \mathscr{H}$, for which $\langle\Phi, E F \Psi\rangle=\|E F\| \equiv \eta$ is real and attains its maximum. Clearly, we must have $E \Phi=\Phi$ and $F \Psi=\Psi$. For fixed $\Psi, E \mathscr{H} \ni \varphi \mapsto\langle\varphi, E \Psi\rangle$ attains its maximum only when $\varphi$ is a positive multiple of $E \Psi$. Hence $E \Psi=\eta \Phi$, and $F \Phi=\eta \Psi$. Consider now the vectors $\Phi^{\prime}=\eta \Phi-\Psi$ and $\Psi^{\prime}=\eta \Psi-\Phi$. These satisfy $E \Phi^{\prime}=F \Psi^{\prime}=0$, and $\left\|\Phi^{\prime}\right\|^{2}=\left\|\Psi^{\prime}\right\|^{2}=1-\eta^{2}$. Moreover, $\left\langle\Phi^{\prime}, \Psi^{\prime}\right\rangle=\eta^{3}-\eta$. Hence

$$
\begin{aligned}
\|E F\| \cdot\left\|\Phi^{\prime}\right\| \cdot\left\|\Psi^{\prime}\right\| & =\eta\left(1-\eta^{2}\right)=-\left\langle\Phi^{\prime}, \Psi^{\prime}\right\rangle=\left\langle\Phi^{\prime},(\mathbb{1}-E)(\mathbb{1}-F) \Psi^{\prime}\right\rangle \\
& \leqq\|(\mathbb{1}-E)(\mathbb{1}-F)\| \cdot\left\|\Phi^{\prime}\right\| \cdot\left\|\Psi^{\prime}\right\|,
\end{aligned}
$$

and $\|E F\| \leqq\|(\mathbb{1}-E)(\mathbb{1}-F)\|$. The reversed inequality follows by exchanging $E \leftrightarrow(\mathbb{1}-E)$ and $F \leftrightarrow(\mathbb{1}-F)$.

(2) Since $E \vee F=\mathbb{1}$ and $E \wedge F=0$, any vector in $\mathscr{H}$ can be written uniquely as $\varphi+\psi$ with $E \varphi=\varphi, F \psi=\psi$. Consider the eigenvalue equation

$$
(E+F)(\varphi+\psi)=(1-\alpha)(\varphi+\psi) \text {. }
$$


Then by uniqueness of the decomposition we must have $E(\varphi+\psi)=(1-\alpha) \varphi$, i.e. $E \psi=-\alpha \varphi$, and, similarly, $F \varphi=-\alpha \psi$. Taking the inner product of the first equation with $\psi$ and of the second with $\varphi$, we get $\langle\varphi, \psi\rangle=-\alpha\|\varphi\|^{2}=\langle\psi, \varphi\rangle=$ $-\alpha\|\psi\|^{2}$. Hence $\alpha\|\varphi\| \cdot\|\psi\|=-\langle\varphi, \psi\rangle=-\langle\varphi, E F \psi\rangle \leqq\|E F\| \cdot\|\varphi\| \cdot\|\psi\|$. Thus $\alpha \leqq\|E F\|$, and $(E+F) \geqq(1-\|E F\|) \mathbb{1}$. Squaring the last inequality we get $E F+F E=(E+F-\mathbb{1})(E+F) \geqq-\|E F\|(E+F)$.

Combining the two parts of the proof, it is clear that the eigenvector of $E+F$ with smallest eigenvalue is $\Phi+\Psi$.

Let $h$ be a VBS interaction in the sense of Definition (5.8) which has a unique zero energy $C^{*}$-finitely correlated ground state $\omega_{0}$ generated by a triple $(\mathbb{E}, \rho, \mathbb{1})$. The state $\omega_{0}$ is purely generated and exponentially clustering. Let $0 \leqq \lambda<1$ denote the minimal rate of decay of correlations in the state $\omega_{0}$, i.e. $\lambda$ is the absolute value of the second largest eigenvalue of $\hat{\mathbb{E}}$. There exists a constant $c>0$ such that

$$
\operatorname{Tr}(\rho)^{-1}\left\|\hat{\mathbb{E}}^{n}-\hat{\mathbb{E}}^{\infty}\right\| \leqq c \lambda^{n} .
$$

As in Definition 5.4 we write $\ell_{0}$ for the interaction length of $\omega$. We will denote the gap in the spectrum of a local Hamiltonian $H_{\{1, \ldots, n\}}$ by $\gamma_{n}$. Finally we denote by $\ell$ the smallest integer for which the intersection property of Lemma 5.5 holds. It follows that $\ell$ is either $\ell_{0}$ or $\ell_{0}+1$. From the remarks at the end of Sect. 5 we can assume that $h$ has range $\ell$, that is $h \in \mathscr{A}^{\otimes \ell}$. We can now estimate the gap of such a VBS model:

6.4 Theorem. With the same notations of above the gap-inequality (6.4) is satisfied for some strictly positive $\gamma$, which can be estimated from below by:

$$
\gamma \geqq \frac{1}{2} \gamma_{2 p}\left(1-2 c \lambda^{p} \frac{1+c \lambda^{p}}{1-c \lambda^{p}}\right)
$$

for $p \geqq \ell$.

Proof. The idea of the proof is the following. First we estimate from below the local Hamiltonian $H_{\{1, \ldots, m p\}}$ by a Hamiltonian $\widetilde{H}_{\{1, \ldots, m\}}$ with the same ground state space: take $p \geqq \ell$ and put

$\tilde{h}_{i, i+1}=H_{\{p i+1, \ldots, p(i+2)\}}=h_{\{p i+1, \ldots, p i+\ell\}}+h_{\{p i+2, \ldots, p i+\ell+1\}}+\cdots h_{\{p(i+2)-\ell+1, \ldots, p(i+2)\}}$ and define

$$
\tilde{H}_{\{1, \ldots, m\}}=\sum_{i=1}^{m-1} \tilde{h}_{i, i+1} .
$$

As $h$ is positive we have the following inequality

$$
H_{\{1, \ldots, m p\}} \geqq \frac{1}{2} \tilde{H}_{\{1, \ldots, m\}} \text {. }
$$

$\tilde{H}_{\{1, \ldots, m\}}$ has to be considered as a nearest neighbor VBS model on an interval $\{1, \ldots m\}$ of a regrouped chain where the one-site algebra in now $\mathscr{A}^{\otimes p}$. Next the positive operator $h$ can be bounded from below by a multiple of the projection $\mathbb{1}-G_{2 p}$ with the same kernel, i.e.

$$
\tilde{h} \geqq \gamma_{2 p}\left(\mathbb{1}-G_{2 p}\right) .
$$


We now estimate the gap of the equivalent Hamiltonian

We will prove

$$
K_{\{1, \ldots, m\}}=\sum_{i=1}^{m-1}\left(\mathbb{1}-G_{2 p}\right)_{i, i+1} .
$$

$$
\left(K_{\{1, \ldots, m\}}\right)^{2} \geqq\left(1-2 c \lambda^{p} \frac{1+c \lambda^{p}}{1-c \lambda^{p}}\right) K_{\{1, \ldots, m\}} .
$$

Combining the inequalities (6.6)-(6.8) we obtain the inequality stated in the theorem. This estimate becomes strictly positive for $p$ large enough. Using Lemma 6.1 we have therefore shown the existence of a non-zero spectral gap in the sense of (6.4).

It remains to prove (6.8). So we have to find a lower bound for the sum of the $(m-1)^{2}$ terms in $\left(K_{\{1, \ldots, m\}}\right)^{2}$. The sum of the diagonal terms in this square just reproduces $K_{\{1, \ldots, m\}}$. Since $\left(\mathbb{1}-G_{2 p}\right)_{i, i+1}$ and $\left(\mathbb{1}-G_{2 p}\right)_{j, j+1}$ commute for $|i-j|>1$, we can bound the sum of all such cross terms by zero. To the projections $E \equiv\left(\mathbb{1}-G_{2 p}\right)_{i, i+1}$ and $F \equiv\left(\mathbb{1}-G_{2 p}\right)_{j, j+1}$ with $|i-j|=1$ we apply successively Lemmas 6.3.(2), 6.3.(1), 5.5, and 6.2, obtaining

$$
\begin{aligned}
E F+F E & \geqq-\|E F-E \wedge F\|(E+F) \\
& =-\left\|\left(G_{2 p} \otimes \mathbb{1}_{p}\right)\left(\mathbb{1}_{p} \otimes G_{2 p}\right)-\left(G_{2 p} \otimes \mathbb{1}_{p}\right) \wedge\left(\mathbb{1}_{p} \otimes G_{2 p}\right)\right\|(E+F) \\
& \geqq-a(p) \frac{1+a(p)}{a_{-}(p)}(E+F) \geqq-c \lambda^{p} \frac{1+c \lambda^{p}}{1+c \lambda^{p}}(E+F) .
\end{aligned}
$$

Since each $\left(\mathbb{1}-G_{2 p}\right)_{i, i+1}$ occurs in at most two of these cross terms, we get (6.8).

Example 8. As a matter of illustration we evaluate the estimate for the gap in the case of the AKLT model. $\lambda$ and $c$ of the theorem are easily determined: $\lambda=\frac{1}{3}$, $c=4$. Hence the smallest value for $p$ leading to a non-trivial estimate is $p=3$. So we need the finite volume gap for six sites. In [50] the value $\gamma_{6}=.398451$ is given. Combining these numbers we find $\gamma \geqq .119$.

As such Theorem 6.4 is not applicable to VBS models where the ground state is not unique. We believe however that for VBS models with a finite ground state degeneracy, the above arguments can be modified to obtain the existence of a spectral gap. In the Examples two such models have been mentioned: the Majumdar-Ghosh model for which the existence of a gap has been obtained in [5], and the model introduced in Example 1 with $\theta=0$ where the existence of a spectral gap is also obvious.

Of course the theorem cannot be applied to the Heisenberg ferromagnet which does not have a gap, simply because it is not a VBS model as was shown in Example 5.

\section{Applications}

7.1 Classical Systems. In this section we consider $C^{*}$-finitely correlated states for which both algebras $\mathscr{A}$ and $\mathscr{B}$ are abelian and finite-dimensional. Hence $\mathscr{A}=\mathscr{C}(\Omega)$ is the set of complex valued functions on a finite set $\Omega$, say $\Omega=\{1, \ldots d\}$. Thus as 
a vector space $\mathscr{A}$ is just $\mathbb{C}^{d}$, and its hermitian part $\mathbb{R}^{d}$ is ordered componentwise. The projections $e_{i} \in \mathscr{A}$ with $e_{i}(j)=\delta_{i j}$ obviously form a basis of $\mathscr{A}$. Similarly, $\mathscr{B} \equiv \mathscr{C}(\{1, \ldots k\})$ for some $k<\infty$. The map $\mathbb{E}: \mathscr{A} \times \mathscr{B} \rightarrow \mathscr{B}$ is best decomposed into the $d$ operators $\mathbb{E}_{i}: \mathscr{B} \mapsto \mathbb{E}\left(e_{i} \otimes B\right)$. Since a map from or into an abelian $C^{*}$-algebra is completely positive iff it is positive [60], this constraint on $\mathbb{E}$ just means that each $\mathbb{E}_{i}$, written as a $k \times k$-matrix with respect to the canonical basis of $\mathscr{B} \equiv \mathbb{C}^{k}$ has positive matrix elements. In order to get a $C^{*}$-finitely correlated state we further need a vector $e \in \mathscr{B}=\mathbb{C}^{k}$ with positive components (which we can take as $\mathbb{1}$ by Lemma 2.5), and another vector $\rho$ with positive components. With the notations $\langle\cdot, \cdot\rangle$ for the scalar product of $\mathbb{C}^{k}, X^{\top}$ for the transpose in $\mathscr{M}_{k}$, and $\hat{\mathbb{E}}=\sum_{i=1}^{d} \mathbb{E}_{i}$ these objects have to satisfy $\hat{\mathbb{E}} e=e$, and $\hat{\mathbb{E}}^{\top} \rho=\rho$.

In probability theory a state on the chain $\mathscr{C}(\Omega)_{\mathbb{Z}}$ is usually called a "stochastic process" with state space $\Omega$, and the state is usually expressed via the Riesz representation theorem as a cylinder measure $\mu$ on $\Omega^{\mathbb{Z}}$. In our construction this measure is given by

$$
\mu\left(\left\{k_{n}, \ldots, k_{m}\right\}\right)=\left\langle\rho, \mathbb{E}_{k_{n}} \cdots \mathbb{E}_{k_{m}} e\right\rangle,
$$

where $\left\{k_{n}, \ldots, k_{m}\right\}$ denotes the cylinder in $\Omega^{\mathbb{Z}}$ consisting of those configurations of the chain that coincide with $\left\{k_{n}, \ldots, k_{m}\right\}$ at the sites $\{n, n+1, \ldots m\}$. We shall also call $\mu$ a $C^{*}$-finitely correlated measure (in [28] these were called "manifestly positive").

It is straightforward to see that any finite state space $m$-step Markovian measure is manifestly positive and finitely correlated. We demonstrated in Sect. 4 that a general $C^{*}$-finitely correlated state can be obtained from a purely generated $C^{*}$-finitely correlated state by embedding the one-site algebra of the given state homomorphically into the one-site algebra of the purely generated state. In the context of classical theories such homomorphisms are induced by continuous mappings between configuration spaces. In probabilistic terminology one process is a "function" of the other. We can now state the following result:

7.1 Theorem. Let $\mu$ be a $C^{*}$-finitely correlated measure on $\Omega^{\mathbb{Z}}$. Then there exists a finite set $\Omega_{1}$, a Markovian measure $\mu_{1}$ on $\Omega_{1}^{\mathbb{Z}}$ and a function $\Phi: \Omega \rightarrow \Omega_{1}$ such that $\mu=\mu_{1} \circ \Phi^{\mathbb{Z}}$. Moreover we can choose $\Omega_{1}$ in such a way that \# $\Omega_{1} \leqq(\# \Omega)^{4}$.

Our next aim is to give an expression for the entropy density of the measure. Such an expression has been obtained by [15] and was extensively studied in [28]. For technical convenience we assume the rather strong irreducibility condition that all matrix elements of the $\mathbb{E}_{k},\{k=1, \ldots d\}$ are strictly positive. This implies that $\hat{\mathbb{E}}$ has trivial peripheral spectrum, and hence that the measure $\mu$ has no non-trivial periodic components. Much weaker conditions are discussed in [28]. We first introduce a dynamical system for the purpose of describing the structure of the "conditionings" of the process $\mu$. So let us denote by $\mathscr{B}_{e}$ the set of positive elements $v$ in $\mathbb{C}^{k}$ such that $\langle v, e\rangle=1$. Thus if we take $e=\mathbb{1}_{\mathscr{B}}$, as we may, $\mathscr{B}_{e}$ is just the state space of $\mathscr{B}$.

An operator $T_{\mu}$ is now defined on the space $\mathscr{C}\left(\mathscr{B}_{e}\right)$ of continuous complex-valued functions on $\mathscr{B}_{e}$ :

$$
\left(T_{\mu} f\right)(v)=\sum_{a \in \Omega}\left\langle v, \mathbb{E}_{a} e\right\rangle f\left(\Gamma_{a}(v)\right) \quad f \in \mathscr{C}\left(\mathscr{B}_{e}\right),
$$


where $\Gamma_{a}: \mathscr{B}_{e} \rightarrow \mathscr{B}_{e}$ is defined by

$$
\Gamma_{a}(v)=\frac{\mathbb{E}_{a}^{\top} v}{\left\langle v, \mathbb{E}_{a} e\right\rangle}
$$

7.2 Theorem. With the above notations there exists a unique probability measure $\bar{\varphi}$ on $\mathscr{B}_{e}$, which is invariant under $T_{\mu}$. The mean entropy $s(\mu)$ of the measure $\mu$ is given by:

$$
s(\mu)=\sum_{a \in \Omega} \int_{\mathscr{B} \boldsymbol{e}} \bar{\varphi}(d v) h_{a}(v),
$$

where $h_{a}(v)=-\left\langle v, \mathbb{E}_{a} e\right\rangle \log \left\langle v, \mathbb{E}_{a} e\right\rangle$.

The $C^{*}$-finitely correlated states described in this section may, of course, be used to generate finitely correlated states on chains $\tilde{\mathscr{A}}_{\mathbf{Z}}$ with non-commutative $\tilde{\mathscr{A}}$ by applying a completely positive map $\mathbb{F}: \mathscr{C}(\Omega) \rightarrow \tilde{\mathscr{A}}$ at each site. These $C^{*}$-finitely correlated states, which could be called non-classical functions of Markov processes, exhaust only a small subset of the $C^{*}$-finitely correlated states. In such a state the correlations across any bond will be "classically correlated" in the sense of [64], i.e. the state can be decomposed as an integral over states, in which the right and left halves of the chain are completely uncorrelated. It is easy to see that non-trivial purely generated states, as studied in Sects. 5 and 6 cannot have this property.

It would be interesting to have examples for states over a classical chain $(\mathscr{A}$ abelian), generated with a non-abelian algebra $\mathscr{B}$. More generally, one might look for finitely correlated states over a classical chain, which are not even $C^{*}$-finitely correlated. We did not succeed in settling the question whether this is possible.

7.2. Integrable Systems. Since $C^{*}$-finitely correlated states are easy to construct, it is natural to use them as trial states in the ground state variational problem of a given interaction. Here we prove a general result, which illuminates the nature of this variation. It also allows a neat one-line proof of the fact that the ground state of the antiferromagnetic spin 1/2 Heisenberg chain with nearest neighbor interaction $h=\sum_{\mu=1}^{3} \sigma^{\mu} \otimes \sigma^{\mu} \in \mathscr{M}_{2} \otimes \mathscr{M}_{2}$ and of some of its generalizations $[63,11]$ are not $C^{*}$-finitely correlated.

7.3 Proposition. Let $h \in\left(\mathscr{M}_{d}\right)^{\otimes \ell}$ be hermitian, and suppose that with respect to some basis $\left\{\varphi_{\mu}\right\}_{\mu=1}^{d}$ the real and imaginary parts of all matrix elements

$$
\left\langle\varphi_{\mu_{1}} \otimes \cdots \varphi_{\mu_{n}}, h \varphi_{v_{1}} \otimes \cdots \varphi_{v_{n}}\right\rangle
$$

are in some subfield $\mathbb{F} \subset \mathbb{R}$. Suppose that $h_{\min } \equiv \inf \{\omega(h) \mid \omega \in \mathscr{T}\}$ is attained at a $C^{*}$-finitely correlated state. Then $h_{\min }$ is algebraic over $\mathbb{F}$.

Proof. We may suppose that the minimizing state $\omega$ is generated by $\mathbb{E}: \mathscr{M}_{d} \otimes \mathscr{M}_{k} \rightarrow \mathscr{M}_{k}$ and $\rho: \mathscr{M}_{k} \rightarrow \mathbb{C}$. In particular, this state has minimal energy density among all states generated by different maps $\mathbb{E}, \rho$ acting on the same spaces. We have to show that minimizing the energy functional over this set leads to an algebraic minimal value. 
Since $\left\{\pi\left(\mathscr{M}_{d}\right) \otimes \mathscr{M}_{k} V \mathbb{C}^{k}\right\}$ is total in the Stinespring dilation space $\mathscr{H} \otimes \mathbb{C}^{k}$ of $\mathbb{E}, \mathscr{H}$ has at most dimension $d^{2} k^{2} \cdot k<\infty$. We may therefore fix a sufficiently large dimensional space $\mathscr{H}$ and a representation $\pi: \mathscr{M}_{d} \rightarrow \mathscr{H}$, and the map $V$ of Proposition 4.2(1) and a matrix $R \in \mathscr{M}_{k}$, with $\rho(B)=\operatorname{Tr}\left(B R^{*} R\right)$ to parametrize all $C^{*}$-finitely correlated states generated in $\mathscr{M}_{k}$.

Using this parametrization there are no positivity constraints, but only the constraints $\mathbb{E}(\mathbb{1})=\mathbb{1}$, and $\rho(\mathbb{E}(\mathbb{1} \otimes B)=\rho(B)$, which are a set of polynomial identities with integer coefficients in the (real and imaginary parts) of the matrix elements of $V$ and $R$. The energy functional

$$
(V, R) \mapsto \operatorname{Tr}\left(R \mathbb{E}^{(\ell)}\left(h \otimes \mathbb{1}_{\mathscr{M}_{k}}\right) R^{*}\right)
$$

is a polynomial of degree 2 in $R$ and degree $2 \ell$ in $V$, with coefficients in $\mathbb{F}$. Since the constraints force $V$ and $R$ to lie in given compact sets, minimizers of the constrained variational problem exist. Introducing as additional variables the Lagrange multipliers $\lambda_{i}$ for the constraints, we obtain a system of polynomial equations for the minimizing $(V, R, \lambda)$. We can cut down the set of minimizing $(V, R, \lambda)$ by further arbitrary polynomial conditions (with coefficients in $\mathbb{F})$, until we have one isolated solution of a system of algebraic equations, which represents a minimizer. We can separate this solution from possible further solutions of the same system (which might not minimize the energy) by some polynomial inequalities. The resulting system of polynomial equations and inequalities thus has a unique solution in the real field. By Tarski's Theorem [45, Sect. 5.6] we can find a set of integer polynomial conditions on the coefficients of all these polynomials, which decides the existence of solutions of the system for any real closed field. Since there is a solution in real variables, this condition is satisfied for the given coefficients. Hence there must also be a solution in the real closed extension of $\mathbb{F}$, i.e. the unique solution is algebraic over $\mathbb{F}$. Therefore also the value of the energy functional must be algebraic.

Recently the exact ground state energy density has been computed for a class of models generalizing the usual spin $1 / 2$ Heisenberg antiferromagnet $[63,11]$. These models are spin $J$ chains with isotropic nearest neighbor Hamiltonians and the matrix elements of the interaction are algebraic numbers.

It follows from the computations that the ground state energy density $e_{0}$ is given by:

$$
\begin{aligned}
& e_{0}=-\sum_{k=0}^{J-1} \frac{1}{2 k+1} \text { for integer } J \\
& e_{0}=-\log 2-\sum_{k=1}^{J-1 / 2} \frac{1}{2 k} \text { for half-integer } J
\end{aligned}
$$

Applying Proposition 7.3 we therefore have:

7.4 Corollary. The ground state of the spin $\frac{1}{2}$ Heisenberg antiferromagnet and of its generalizations $[63,11]$ to higher half-integer spins is not finitely correlated.

7.3 Gauge Invariant States. It is clear that under suitable covariance conditions $\mathbb{E}$ and $\rho$ will generate a state $\omega$, which is invariant under the action of some gauge 
group $G$. For simplicity, let us take $\mathscr{A}=\mathscr{M}_{d}$ and consider two additional Hilbert spaces $\mathscr{K}$ and $\mathscr{K}^{\prime}$. Let $\mu, \lambda$ and $\lambda^{\prime}$ denote three unitary representations of $G$ on $\mathbb{C}^{d}, \mathscr{K}$ and $\mathscr{K}^{\prime}$ respectively. We suppose that there exists a non-zero intertwining isometry $V: \mathscr{K} \rightarrow \mathbb{C}^{d} \otimes \mathscr{K}^{\prime} \otimes \mathscr{K}$ satisfying

$$
V \lambda(g)=\left(\mu(g) \otimes \lambda^{\prime}(g) \otimes \lambda(g)\right) V
$$

for all $g \in G$. Set $\mathscr{B}=\mathscr{B}(\mathscr{K}), \mathbb{E}(A \otimes B)=V^{*}\left(A \otimes \mathbb{1}_{\mathscr{K}} \otimes B\right) V, e=\mathbb{1} \in \mathscr{B} \equiv \mathscr{B}(\mathscr{K})$, and choose an $\hat{\mathbb{E}}$-invariant state $\rho$ on $\mathscr{B}(\mathscr{K})$, e.g. the normalized trace. Then $\hat{\mathbb{E}}$ satisfies the covariance relation: $\lambda(g) \mathbb{E}(X) \lambda(g)^{*}=\mathbb{E}\left((\mu(g) \otimes \lambda(g)) X(\mu(g) \otimes \lambda(g))^{*}\right)$ From $(2.1)$ it then follows that $(\mathbb{E}, \rho, e)$ generates a state $\omega$, which is invariant under the gauge group $G$ :

$$
\omega\left(A_{m} \otimes \cdots A_{n}\right)=\omega\left(\mu(g) A_{m} \mu(g)^{*} \otimes \cdots \mu(g) A_{n} \mu(g)^{*}\right) .
$$

If the map $\mathbb{E}$ is pure then $\mathscr{K}^{\prime}$ is one-dimensional. Thus, in order to construct purely generated gauge invariant states by this formula, we only have to pick the representations $\lambda$ and $\mu$. Note that the intertwining relation does not automatically imply that $\hat{\mathbb{E}}$ has only one fixed vector, so this condition has to be checked by hand. If it is satisfied, however, the theory of Sects. 5 and 6 applies. It can be shown that the scheme of above is essentially the only possibility for constructing $C^{*}$-finitely correlated states invariant under a local gauge group [33].

In the AKLT model (see Example 1) the gauge group is $S U(2) . \mathscr{B}=\mathscr{M}_{2}$, $\mathscr{A}=\mathscr{M}_{3}$ and $\lambda$ and $\mu$ are the irreducible representations of $S U(2)$ on $\mathbb{C}^{2}$ and $\mathbb{C}^{3}$ respectively. This determines uniquely the intertwiner $V$. We will now study a generalization of this example to arbitrary integer spin.

Let $\mathscr{A}=\mathscr{M}_{2 J+1}$, where $J$ is the value of the spin at each site. $J$ is assumed to be integer for reasons that will become apparent immediately. The algebra $\mathscr{B}$ will be chosen as $\mathscr{M}_{2 j+1}$ for some not necessarily integer $j \in \frac{1}{2} \mathbb{N}$, satisfying $j \geqq J / 2$. These are precisely the constraints on $j$ and $J$ for an interwining operator

$$
V: \mathbb{C}^{2 j+1} \rightarrow \mathbb{C}^{2 J+1} \otimes \mathbb{C}^{2 j+1} \quad \text { with } \quad\left(\mathscr{D}_{g}^{(J)} \otimes \mathscr{D}_{g}^{(j)}\right) V=V \mathscr{D}_{g}^{(j)}
$$

to exist. In this case $V$ is unique up to a scalar factor, and we can, and will choose this factor so that $V$ is an isometry. Then $\mathbb{E}: \mathscr{A} \otimes \mathscr{B} \rightarrow \mathscr{B}$, given by $\mathbb{E}(X)=V^{*} X V$ is completely positive and unit preserving. Let us denote by $\tau$ the normalized trace on $\mathscr{B}$, which is the only rotation invariant state on that algebra. Since $\hat{\mathbb{E}}$ obviously maps rotation invariant into rotation invariant states, it is clear that $\tau \circ \hat{\mathbb{E}}=\tau$. Consequently, $(\mathbb{E}, \tau, \mathbb{1})$ generate a $S U(2)$-invariant $C^{*}$-finitely correlated state $\omega_{j}$. Note also that $\mathbb{E}$ is pure, so $\omega_{j}$ is purely generated, and since the eigenvalue 1 of $\hat{\mathbb{E}}$ is non-degenerate (see below) the whole theory of Sects. 5 and 6 applies.

7.5 Proposition. Any correlation function $n \in \mathbb{N} \mapsto c(n) \equiv \omega_{j}\left(X_{1} \alpha_{n}\left(X_{2}\right)\right)$ with $X_{\#} \in \mathscr{A}_{\mathbb{N}}$, $X_{b} \in \mathscr{A}_{\mathbb{Z} \backslash \mathbb{N}}$ is of the form $c(n)=\sum_{k=0}^{2 j} a_{k} \lambda_{k}^{n}$ for some constants $a_{k}$, where $\lambda_{k}$ is the $k^{\text {th }}$ eigenvalue of $\hat{\mathbb{E}} . \lambda_{k}$ is $(2 k+1)$-fold degenerate, and equal to

$$
\lambda_{k}=(-1)^{k}(2 j+1)\left\{\begin{array}{lll}
j & j & J \\
j & j & k
\end{array}\right\} \quad k=0, \ldots 2 j,
$$

where the symbol between braces is a Wigner 6j-symbol using the conventions of [26]. 
Proof. In order to get at the behavior of the correlation functions we must diagonalize $\hat{\mathbb{E}}$. There is a natural identification of the $k \times k$ matrices $\mathscr{M}_{k}$ with $\mathbb{C}^{k} \otimes \mathbb{C}^{k}$ : the rank 1 operator $|\psi\rangle\langle\varphi|$ is mapped onto $\psi \otimes \bar{\varphi}$ where $\varphi \mapsto \bar{\varphi}$ is a complex conjugation on $\mathbb{C}^{k}$. This is in fact a unitary transformation if we equip $\mathscr{M}_{k}$ with the Hilbert-Schmidt inner product $\langle A, B\rangle \equiv \operatorname{Tr} A^{*} B$. The representation $g \in S U(2) \mapsto \alpha_{g}^{(k)}(\cdot) \equiv \mathscr{D}_{g}^{(k)} \cdot \mathscr{D}_{g}^{(k)^{*}}$ in the automorphisms of $\mathscr{M}_{k}$ is transported by this unitary transformation into the representation $g \in S U(2) \mapsto \mathscr{D}_{g}^{(k)} \otimes \bar{D}_{g}^{(k)}$, where $\overline{\mathscr{D}_{g}^{(k)}} \varphi \equiv \overline{\mathscr{D}_{g}^{(k)} \bar{\varphi}}$ but, as there is up to unitary equivalence only one irreducible spin $k$ representation of $S U(2), \mathscr{D}^{(k)}$ and $\overline{\mathscr{D}^{(k)}}$ are unitarily equivalent.

As we have to consider decompositions of tensor representations of $S U(2)$ we recall the usual conventions [26]:

- $\{|k, m\rangle \mid m=-k,-k+1, \ldots k\}$ denotes the standard basis of $\mathbb{C}^{2 k+1}$ which corresponds to the spin $k$ representation of $S U(2):|k, m\rangle$ is the normalized eigenvector of the $z$-component of the spin corresponding to the eigenvalue $m$ and the successive $|k, m\rangle$ are obtained by applying the lowering operator to the highest spin vector $|k, k\rangle$ and normalizing with a positive factor.

- $\left|\left(k_{1}, k_{2}\right) k_{3}, m_{3}\right\rangle$ denotes the $\left|k_{3}, m_{3}\right\rangle$ vector in the spin $k_{3}$ subrepresentation of $\mathscr{D}^{\left(k_{1}\right)} \otimes \mathscr{D}^{\left(k_{2}\right)}$. The overall phase in each $\mathscr{D}^{\left(k_{3}\right)}$ subrepresentation is fixed by requiring that $\left|k_{1}, k_{1}\right\rangle \otimes\left|k_{2}, k_{3}-k_{1}\right\rangle$ appears with a positive coefficient in $\left|\left(k_{1}, k_{2}\right) k_{3}, k_{3}\right\rangle$.

As $V$ intertwines $\mathscr{D}^{(j)}$ and $\mathscr{D}^{(J)} \otimes \mathscr{D}^{(j)}$ we have with the notations of above that the matrix elements of $V$ are precisely the Clebsch-Gordan coefficients:

$$
\begin{aligned}
\left\langle J, m_{1}\right| \otimes\left\langle j, m_{2}|V| j, m_{1}+m_{2}\right\rangle & =\left\langle J, m_{1}\right| \otimes\left\langle j, m_{2} \mid(J, j) j, m_{1}+m_{2}\right\rangle \\
& \equiv\left\langle J, m_{1}, j, m_{2} \mid(J, j) j, m_{1}+m_{2}\right\rangle .
\end{aligned}
$$

As $\hat{\mathbb{E}} \circ \alpha_{g}^{(j)}=\alpha_{g}^{(j)} \circ \hat{\mathbb{E}}, \hat{\mathbb{E}}$ will be constant on each of the subspaces of $\mathscr{M}_{2 j+1}$ that carries an irreducible subrepresentation of $\alpha_{g}^{(j)}$. Using the identifications of above the spectrum of $\hat{\mathbb{E}}$ consists of eigenvalues $\left\{\lambda_{k} \mid k=0,1, \ldots 2 j\right\}$, and the multiplicity of $\lambda_{k}$ is $2 k+1$ which is the dimension of the spin $k$ irreducible representation of $\alpha_{g}^{(j)}$. In order to compute the values of the $\lambda_{k}$ it is useful to make the following explicit choice for the complex conjugation:

$$
\overline{|k, m\rangle} \equiv(-1)^{k-m}|k,-m\rangle \quad m=-k,-k+1, \ldots k .
$$

With this choice $\overline{\mathscr{D}_{g}^{(k)}}=\mathscr{D}_{g}^{(k)}$. As $\mathbb{1} \in \mathscr{M}_{2 J+1}$ carries the spin 0 subrepresentation of $\alpha_{g}^{(J)}$ and has Hilbert-Schmidt norm $\sqrt{1+2 J}$ it can be identified with $\sqrt{2 J+1}$ $|(J, J) 0,0\rangle$ also the spin $k$ subspace of $\mathscr{M}_{2 j+1}$ is generated by $\{|(j, j) k, m\rangle \mid m=$ $-k,-k+1, \ldots k\}$. It is now straightforward to write down the eigenvalue equation for $\hat{\mathbb{E}}$ and to compute the $\lambda_{k}$ using the conventions of [26]. As the eigenvalue 1 is non-degenerate $\omega_{j}$ is pure.

We can now proceed to construct interactions exposing these states. Restricting, for simplicity, to the case $j \leqq J \leqq 2 j$, it is not difficult to see that the range of $\Gamma_{2}$ has its maximal value $k^{2}=(2 j+1)^{2}$. Hence by Definition 5.4 the interaction length of all these states is 2 , and we know that we can find exposing interactions in $\mathscr{A}^{\otimes 3}$, i.e. an exposing next-nearest neighbor interaction. When $j<J, \mathscr{G}_{2}$ is a proper subspace of $\mathscr{H}^{\otimes 2}$. This subspace is easily described in terms of the representation theory. Given two representations $\mathscr{D}^{\left(s_{2}\right)}, i=1,2$, let us denote by $\mathscr{R}_{s_{1}, s_{2}}^{s}$ the subspace 
of $\mathbb{C}^{2 s_{2}+1} \otimes \mathbb{C}^{2 s_{2}+1}$ carrying representations with spin less than or equal to $s$, and, similarly, denote by $\mathscr{R}_{s_{1}, s_{2}, s_{3}}^{s_{3}}$ the subspace of $\mathbb{C}^{2 s_{1}+1} \otimes \mathbb{C}^{2 s_{2}+1} \otimes \mathbb{C}^{2 s_{3}+1}$ with spin $\leqq s$. Then since $V^{(2)} \stackrel{s_{1}, s_{2}, s_{3}}{=}(\mathbb{1} \mathscr{H} \otimes V) V: \mathscr{K} \rightarrow \mathscr{H} \otimes \mathscr{H} \otimes \mathscr{K}$ intertwines $\mathscr{D}^{(j)}$ with $\mathscr{D}^{(J)} \otimes \mathscr{D}^{(J)} \otimes \mathscr{D}^{(j)}$, it is clear that $V^{(2)} \mathscr{K} \subset \mathscr{R}_{J J}^{2 j}$. Similarly, $V^{(3)} \mathscr{K} \subset \mathscr{R}_{J J J}^{2 j}$. Thus if $P_{2}^{s}$ denotes the projection on $\mathscr{H} \otimes \mathscr{H}$ onto the subspace carrying the spin $s$ representation, we have $\omega_{j}\left(\alpha_{i}\left(k^{j}\right)\right) \equiv 0$ with $k^{j} \in \mathscr{A}^{\otimes 2}$ given by

$$
k^{j}=\sum_{s=2 j+1}^{2 J} P_{2}^{s} .
$$

Note that $k^{j}$ cannot be an exposing interaction for $j>J / 2$, since also $\omega_{J / 2}\left(\alpha_{i}\left(k^{j}\right)\right) \equiv 0$, contradicting the uniqueness theorem 5.7. However, for the smallest possible value $j=J / 2, h=k^{j}$ is indeed an interaction exposing $\omega_{j}$. This reduction from a next-nearest neighbor to a nearest neighbor interaction follows from the following proposition (inserting $s_{i}=s_{i j}=J$ ), which is a direct application of the technique used in $[9,46]$.

7.6 Proposition. Let $s_{1}, s_{2}, s_{3}, s_{12}, s_{23} \in \frac{1}{2} \mathbb{N}$. Let $\left(s_{12}-\left|s_{1}-s_{2}\right|\right),\left(s_{23}-\left|s_{2}-s_{3}\right|\right) \in \mathbb{N}$. Then

$$
\mathscr{R}_{s_{1}, s_{2}}^{s_{12}} \otimes \mathbb{C}^{2 s_{3}+1} \cap \mathbb{C}^{2 s_{1}+1} \otimes \mathscr{R}_{s_{2}, s_{3}}^{s_{23}} \subset \mathscr{R}_{s_{1}, s_{2}, s_{3}}^{s_{12}},
$$

provided that $s_{12}+s_{23}-s_{2} \leqq s_{123}$.

Proof. It is most convenient to realize $\mathbb{C}^{2 s+1}$ as the space of complex polynomials in two variables $u$ and $v$, which are homogeneous of degree $2 s$. The elements of $\mathbb{C}^{2 s_{1}+1} \otimes \mathbb{C}^{2 s_{2}+1}$ thus become polynomials in four variables $u_{1}, v_{1}, u_{2}, v_{2}$, and so on for higher tensor products. Then $\psi \in \mathscr{R}_{s_{1}, s_{2}}^{j}$ iff the polynomial $\psi$ can be factorized as

$$
\psi\left(u_{1}, v_{1}, u_{2}, v_{2}\right)=\left(u_{1} v_{2}-v_{1} u_{2}\right)^{s_{1}+s_{2}-j} \varphi\left(u_{1}, v_{1} ; u_{2}, v_{2}\right),
$$

for a polynomial $\varphi$, which is homogeneous of degree $s_{1}-s_{2}+j$ in the variables $\left(u_{1}, v_{1}\right)$, and of degree $s_{2}-s_{1}+j$ in the second set of variables. For a discussion of this structure see $[39$, p. $369 \mathrm{ff}]$. Consider now a polynomials in six variables, which is in the intersection described in the proposition, that is a polynomial with two factorizations

$$
\begin{aligned}
\psi\left(u_{1}, v_{1} ; u_{2}, v_{2} ; u_{3}, v_{3}\right) & =\left(u_{1} v_{2}-v_{1} u_{2}\right)^{s_{1}+s_{2}-s_{12}} \varphi\left(u_{1}, v_{1} ; u_{2}, v_{2} ; u_{3}, v_{3}\right) \\
& =\left(u_{2} v_{3}-v_{2} u_{3}\right)^{s_{2}+s_{3}-s_{23}} \chi\left(u_{1}, v_{1} ; u_{2}, v_{2} ; u_{3}, v_{3}\right),
\end{aligned}
$$

with polynomials $\varphi, \chi$. Clearly the factors $\left(u_{1} v_{2}-v_{1} u_{2}\right)$ cannot be further factorized into polynomials. Hence by the prime factorization theorem for many variablepolynomials $[45$, Sect. 2.16$]$ we find that there must be a polynomial $\tilde{\psi}$ such that

$$
\psi\left(u_{1}, \ldots v_{3}\right)=\left(u_{1} v_{2}-v_{1} u_{2}\right)^{s_{1}+s_{2}-s_{12}}\left(u_{2} v_{3}-v_{2} u_{3}\right)^{s_{2}+s_{3}-s_{23}} \tilde{\psi}\left(u_{1}, \ldots v_{3}\right) .
$$

Clearly, $\tilde{\psi}$ is homogeneous of total degree $2\left(s_{1}+s_{2}+s_{3}-\left(s_{1}+s_{2}-s_{12}\right)-\right.$ $\left.\left(s_{2}+s_{3}-s_{23}\right)\right)=2\left(s_{12}+s_{23}-s_{2}\right)$. Consider now a simultaneous transformation of each variable pair by an $S U(2)$-transformation $\left(u_{i}, v_{i}\right) \mapsto\left(a u_{i}+b v_{i},-b^{*} u_{i}+a^{*} v_{i}\right)$ with $a a^{*}+b b^{*}=1$. Since the factor multiplying $\tilde{\psi}$ is invariant under such transformations, this degree is also the homogeneous power, with which $a, a^{*}, b, b^{*}$ appear in the transformed polynomial. That is to say, $\psi$ is supported by the subspace of spins less than $s_{12}+s_{23}-s_{2}$. 
The simplest example of this situation occurs when $J=1$ and $j=1 / 2$. In this case the nearest neighbor interaction $h$ is precisely the AKLT model. For examples of half-integer spin models we refer to [31].

\section{Appendix: Matrix Order and Conditions for Positivity}

The concept of matrix order originated in the theory of operator algebras $[10,23,24,27,57]$. As a starting point one might take the observation that the order structure of a $C^{*}$-algebra almost determines the algebraic structure, in the sense that an order isomorphism between $C^{*}$-algebras can be split in a certain sense into a homomorphism and an antihomomorphism. Antihomomorphisms like the transpose map on a matrix algebra behave strangely also in that the tensor product of such a map with the identity map of another algebra fails to be positive. However, if one imposes on (iso-)morphisms the requirement of "complete positivity," i.e. the stability of positivity under tensoring with identity maps, then "order isomorphism" implies algebraic isomorphism. A matrix ordering of a vector space is just the "enhanced order structure," corresponding to this more restrictive notion of order isomorphism. The reason this structure appears in the present context is that an ordered linear subspace or quotient of a $C^{*}$-algebra automatically inherits a matrix ordering from the algebra, but, unless it is a sub-algebra, it carries no canonical product operation. We now proceed with the formal definitions.

For any complex vector space $\mathscr{B}$, we shall denote by $\mathscr{M}_{n}(\mathscr{B})$ the space of $n \times n$-matrices with entries in $\mathscr{B}$. We shall also identify this space with $\mathscr{M}_{n} \otimes \mathscr{B}$, where we have written $\mathscr{M}_{n}$ for $\mathscr{M}_{n}(\mathbb{C})$. $\mathscr{M}_{n, m}$ will denote the space of complex $n \times m$ matrices $V=\left(V_{i j}\right)_{i=1}^{n}{ }_{j=1}^{m}$, and for any $B \in \mathscr{M}_{n}(\mathscr{B}), V \in \mathscr{M}_{n, m}$ we define $V^{*} B V \in \mathscr{M}_{m}(\mathscr{B})$ by

$$
\left(V^{*} B V\right)_{j j^{\prime}}=\sum_{i, i^{\prime}} \overline{V_{i j}} B_{i i^{\prime}} V_{i^{\prime} j^{\prime}}
$$

When $\mathscr{B}$ has an antilinear involution $B \mapsto B^{*}$, an ordering of $\mathscr{B}$ is defined by a proper generating cone $\mathscr{B}_{+} \subset \mathscr{B}_{h}=\left\{B \in \mathscr{B} \mid B=B^{*}\right\}$, i.e. $\mathscr{B}_{+}$is closed under addition and multiplication with positive scalars, $\mathscr{B}_{+} \cap\left(-\mathscr{B}_{+}\right)=\{0\}$, and $\mathscr{B}_{+}$generates $\mathscr{B}$ as a vector space. $\mathscr{M}_{n}(\mathscr{B})$ will then always be taken with the involution $\left(B^{*}\right)_{i j}=\left(B_{j i}\right)^{*}$. A matrix ordered space $\mathscr{B}$ is by definition a complex vector space with involution, such that every $\mathscr{M}_{n}(\mathscr{B})$ is ordered by a proper generating cone $\mathscr{M}_{n}(\mathscr{B})_{+} \subset \mathscr{M}_{n}(\mathscr{B})_{h}$, and these cones have the property that for all $n, m \in \mathbb{N}, B \in \mathscr{M}_{n}(\mathscr{B})_{+}, V \in \mathscr{M}_{n m}$ we have $V^{*} B V \in \mathscr{M}_{m}(\mathscr{B})_{+}$. A linear map $\mathbb{F}: \mathscr{A} \rightarrow \mathscr{B}$ between matrix ordered spaces is called completely positive, if the maps $\mathbb{F}_{n}=\mathrm{id} \mathscr{M}_{n} \otimes \mathbb{F}$, i.e. the maps defined by $\left(\mathbb{F}_{n} A\right)_{i j}=\mathbb{F}\left(A_{i j}\right), i, j=1, \ldots n$, are positive for all $n$.

If $\mathscr{B}$ is matrix ordered, and $\mathscr{A}$ is a finite-dimensional $C^{*}$-algebra, then $\mathscr{A} \otimes \mathscr{B}$ is matrix ordered in a canonical way: since $\mathscr{A}=\bigoplus_{\alpha} \mathscr{M}_{n_{\alpha}}$, for some finite set of (possibly equal) numbers $n_{\alpha} \in \mathbb{N}$, we can set

$$
\begin{aligned}
\mathscr{M}_{n}(\mathscr{A} \otimes \mathscr{B})_{+} & =\bigoplus_{\alpha}\left(\mathscr{M}_{n} \otimes \mathscr{M}_{n_{\alpha}} \otimes \mathscr{B}\right)_{+} \\
& =\bigoplus_{\alpha}\left(\mathscr{M}_{n \cdot n_{\alpha}}(\mathscr{B})\right)_{+} \cdot
\end{aligned}
$$


Therefore, it makes sense to demand in Proposition 2.3 that $\mathbb{E}: \mathscr{A} \otimes \mathscr{B} \rightarrow \mathscr{B}$ is completely positive.

It is evident that the composition of completely positive maps is completely positive. Moreover, if $\mathbb{F}: \mathscr{B}_{1} \rightarrow \mathscr{B}_{2}$ is completely positive, and $\mathscr{A}$ is a finite-dimensional $C^{*}$-algebra, the map id $\mathscr{A} \otimes \mathbb{F}: \mathscr{A} \otimes \mathscr{B}_{1} \rightarrow \mathscr{A} \otimes \mathscr{B}_{2}$ is completely positive. Note that this is all that is needed for the argument given after Proposition 2.3, which shows that complete positivity of $\mathbb{E}$ is indeed sufficient to ensure positivity of the state generated by $\mathbb{E}$ and positive elements $e \in \mathscr{B}, \rho \in \mathscr{B}^{*}$.

The second direction of Proposition 2.3 is now contained in the following lemma:

A.1 Lemma. Let $\mathscr{A}$ be a finite-dimensional $C^{*}$-algebra, and let $\omega$ be a finitely correlated state on $\mathscr{A}_{\mathbf{Z}}$. Let $\mathscr{B}$ denote the unique minimal space characterized in Proposition 2.1. Then $\mathscr{B}$ can be matrix ordered such that $\mathbb{E}$ is completely positive.

Proof. Clearly, $\mathscr{B}$ inherits an involution from $\mathscr{A}_{\#}$ by setting $[\hat{A}]^{*}=\left[\hat{A}^{*}\right]$. We define $B \in \mathscr{M}_{n}(\mathscr{B})$ to be positive, if there is some $\hat{A} \in \mathscr{M}_{n}\left(\mathscr{A}_{\#}\right)_{+}$, such that $B_{i j}=\left[\hat{A}_{i j}\right]$. Clearly, this defines a generating cone in $\mathscr{M}_{n}(\mathscr{B})_{h}$. It is also proper, because if both $B \in \mathscr{M}_{n}(\mathscr{B})_{+}$and $-B \in \mathscr{M}_{n}(\mathscr{B})_{+}$, we have $A, A^{\prime} \in \mathscr{M}_{n}\left(\mathscr{A}_{\#}\right)$ such that for all $0 \leqq X \in \mathscr{A}_{\{n \mid n \leqq 0\}}$ the $n \times n$-matrix $\omega\left(X \otimes A_{i j}\right)=\Phi_{X}\left(B_{i j}\right)=\omega\left(X \otimes A_{i j}^{\prime}\right)$ is both positive and negative semidefinite. Thus $\Phi_{X}\left(B_{i j}\right)$ vanishes for all positive $X$, hence for all $X \in \mathscr{A}_{\{n \mid n \leqq 0\}}$, hence $B=0$. The compatibility of these cones for different $n$

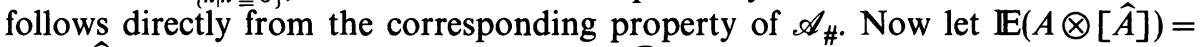
$[A \otimes \hat{A}]$ as in Lemma 1.1 and let $\mathscr{A}=\bigoplus_{\alpha} \mathscr{M}_{n_{\alpha}}$ as above. Then by definition $X=\bigoplus_{\alpha} X^{\alpha} \in \mathscr{M}_{n}(\mathscr{A} \otimes \mathscr{B})=\bigoplus_{\alpha} \mathscr{M}_{n}\left(\mathscr{M}_{n_{\alpha}}(\mathscr{B})\right)$ is positive iff for each $\alpha$ there is a positive $\hat{X}^{\alpha} \in \mathscr{M}_{n}\left(\mathscr{M}_{n_{\alpha}}\left(\mathscr{A}_{\#}\right)\right)$ such that for $i, j=1, \ldots n, \mu, v=1, \ldots n_{\alpha}$ we have $\left(X_{i i}^{\alpha}\right)_{\mu \nu}=\left[\left(\hat{X}_{i i}^{\alpha}\right)_{\mu \nu}\right]$. Hence $\bigoplus_{\alpha} \hat{X}^{\alpha} \in \mathscr{M}_{n}\left(\mathscr{A} \otimes \mathscr{A}_{\#}\right)$ is also positive, and so is its equivalence class $[\hat{X}]=\stackrel{a}{\mathbb{E}}(X)$.

Note that the matrix order for $\mathscr{B}$ is defined completely in terms of $\omega$. This has an important consequence: if there is some automorphism $\alpha$ of $\mathscr{A}$, such that $\omega$ is invariant under sitewise application of $\alpha$, formally $\omega^{\circ} \alpha^{\infty}$, then $\beta([A])=\left[\alpha^{\infty}(A)\right]$ defines an invertible linear map on $\mathscr{B}$. Obviously, $\mathbb{E}(\alpha(A) \otimes \beta(B))=\alpha(\mathbb{E}(A \otimes B))$. And by simply transforming every step in the construction with $\alpha$ or $\beta$, we find that $\beta$ is even completely positive. Clearly, this would be a very useful fact for the discussion of gauge groups, as in Sect. 7.3, were it not for the intractability of the theory of group representations on general matrix ordered spaces.

We remark that some of the results stated in the paper for $C^{*}$-finitely correlated states can be proven for general finitely correlated states as well. Among these are Proposition 2.6, and a variant of Proposition 3.1. However, Proposition 3.3 explicitly uses the product in $\mathscr{B}$, and all of Sect. 4-6 would be very difficult to generalize, since there seems to be no dilation theory for completely positive maps between general matrix ordered spaces.

Acknowledgements. Parts of this work was completed while M.F. visited the Dublin Institute for Advanced Study. It is a pleasure for him to thank DIAS for the warm hospitality. He also acknowledges a helpful discussion with J. Denef on Sect. 7.2. B.N. acknowledges support from 
the Fondo Nacional de Desarrollo Científico y Tecnologico (Chile, Fondecyt project Nr 90-1156). R.F.W. would like to thank the Alexander von Humboldt-Foundation, and the Deutsche Forschungsgemeinschaft for supporting him with fellowships.

\section{References}

1. Accardi, L.: Topics in quantum probability. Phys. Rep. 77, 169-192 (1981)

2. Accardi, L., Frigerio, A.: Markovian Cocycles. Proc. R. Ir. Acad. 83A(2), 251-263 (1983)

3. Affleck, I.: Large- $n$ limit of $S U(n)$ quantum "spin" chains. Phys. Rev. Lett. 54, 966-969(1985)

4. Affleck, I., Lieb, E. H.: A proof of part of Haldane's conjecture on quantum spin chains. Lett. Math. Phys. 12, 57-69 (1986)

5. Affleck, I., Kennedy, T., Lieb, E. H., Tasaki, H.: Valence bond ground states in isotropic quantum antiferromagnets. Commun. Math. Phys. 115, 477-528 (1988)

6. Affleck, I., Lieb, E. H., Kennedy, T., Tasaki, H.: Rigorous results on valence-bond ground states in antiferromagnets. Phys. Rev. Lett. 59, 799-802 (1987)

7. Anderson, $\mathrm{P}$.: The resonating valence bond state in $\mathrm{La}_{2} \mathrm{CuO}_{4}$ and superconductivity. Science 235, 1196-1198 (1987)

8. Araki, H.: Gibbs states of a one-dimensional quantum lattice. Commun. Math. Phys. 14, $120-157$ (1969)

9. Arovas, D. P., Auerbach, A., Haldane, F. D. M.: Extended Heisenberg models of antiferromagnetism: Analogies to the fractional quantum Hall effect. Phys. Rev. Lett. 60, 531-534 (1988)

10. Arveson, W.: Subalgebras of $C^{*}$-algebras. Acta Math. 123, 141-224 (1969)

11. Babujian, H. M.: Exact solution of the one-dimensional isotropic Heisenberg chain with arbitrary spins. Phys. Lett. 90A, 479-482 (1982)

12. Batchelor, M. T., Barber, M. N.: Spin-s quantum chains and Temperley-Lieb algebras. J. Phys. A23, L15-L21 (1990)

13. Batchelor, M.T., Mezincescu, L., Nepmechie, R., Rittenberg, V.: q-deformations of the $O$ (3)symmetric spin 1 chain. J. Phys. A23, L141-L144 (1990)

14. Bethe, H.: Zur Theorie der Metalle. I. Eigenwerte und Eigenfunktionen der linearen Atomkette. Z. Phys. 71, 205-226 (1931)

15. Blackwell, D.: The entropy of functions of finite Markov chains. Transactions of the first Prague conference on information theory, statistical decision functions and random processes. Publishing House of the Czechoslovak Academy of Sciences, Prague 1957

16. Bratteli, O., Robinson, D. W.: Operator algebras and quantum statistical mechanics, 2 vols. Berlin, Heidelberg, New York: Springer 1979 and 1981

17. van den Broeck, P. M.: Exact value of the ground state energy of the linear antiferromagnetic Heisenberg chain with nearest and next-nearest neighbor interactions. Phys. Lett. 77A, 261-262 (1980)

18. Chang, K., Affleck, I., Hayden, G. W., Soos, Z. G.: A study of the bilinear-biquadratic spin 1 antiferromagnetic chain using the valence-bond basis. J. Phys. C1, 153-167 (1989)

19. Caspers, W. J.: Exact ground states for a class of linear antiferromagnetic spin systems. Physica 115A, 275-280 (1982)

20. Caspers, W. J., Magnus, W.: Exact ground states for a class of linear quantum spin systems. Physica 119A, 291-294 (1983)

21. Caspers, W. J., Magnus, W.: Some exact excited states in a linear antiferromagnetic spin system. Phys. Lett. 88A, 103-105 (1982)

22. Chayes, J., Chayes, L., Kivelson, S.: Valence bond ground states in a frustrated two-dimensional spin 1/2 Heisenberg antiferromagnet. Commun. Math. Phys. 123, 53-83 (1989)

23. Choi, M. D.: A Schwarz inequality for positive linear maps on $C^{*}$-algebras. Illinois J. Math. 18, 565-574 (1974)

24. Choi, M. D., Effros, E.: Injectivity and operator spaces. J. Funct. Anal. 24, 156-209 (1977)

25. Dyson, F. J. Lieb, E. H., Simon, B.: Phase transitions in quantum spin systems with isotropic and nonisotropic interactions. J. Stat. Phys. 18, 335-383 (1978) 
26. Edmonds, A. R.: Angular momentum in quantum mechanics. Princeton, NJ: Princeton University Press 1957

27. Effros, E.: "Aspects of non-commutative order." In: Araki, H., Kadison, R. V. (eds). $C^{*}$-algebras and applications to physics. Lect. Notes Math. vol. 650, Berlin Heidelberg New York: Springer 1978

28. Fannes, M., Nachtergaele, B., Slegers, L.: Functions of Markov processes and algebraic measures. Rev. Math. Phys. (in press)

29. Fannes, M., Nachtergaele, B., Werner, R. F.: Exact ground states of quantum spin chains. Europhys. Lett. 10, 633-637 (1989)

30. Fannes, M., Nachtergaele, B., Werner, R. F.: Valence bond states on quantum spin chains as ground states with spectral gap. J. Phys. A, Math. Gen. 24, L185-L190 (1991)

31. Fannes, M., Nachtergaele, B., Werner, R. F.: Entropy estimates for finitely correlated states. Preprint KUL-TF-91/09

32. Fannes, M., Nachtergaele, B., Werner, R. F.: Ground states of VBS models on Cayley trees. J. Stat. Phys. (in press)

33. Fannes, M., Nachtergaele, B., Werner, R. F.: Finitely correlated pure states and their symmetries. In preparation

34. Fannes, M., Verbeure, A.: On solvable models in classical lattice systems. Commun. Math. Phys. 96, 115-124 (1984)

35. Freitag, W.-D., Müller-Hartmann, E.: Complete analysis of two-spin correlations of valence bond solid chains for all integer spins. Z. Physik B 83, 381-390 (1991)

36. Groh, U.: The peripheral point spectrum of Schwarz operators on $C^{*}$-algebras. Math. Z. 176, 311-318 (1981)

37. Hagiwara, M., Katsuma, K., Affleck, I., Halperin, B. I., Renard, J. P.: Observation of $S=1 / 2$ degrees of freedom in an $S=1$ linear chain Heisenberg antiferromagnet. Phys. Rev. Lett. 25, 3181-3184 (1990)

38. Haldane, F. D. M.: Continuum dynamics of the 1-D Heisenberg antiferromagnet: Identification with the $O(3)$ nonlinear sigma model. Phys. Lett. 93A, 464-468 (1983)

39. Hammermesh, M.: Group theory and its applications to physical problems. Reading, Mass.: Addison-Wesley 1962

40. Hudson, R. L., Moody, G. R.: Locally normal symmetric states and an analogue of de Finetti's theorem. Z. Wahrsch. Verw. Gebiete 33, 343-351 (1976)

41. Hulthén, L.: Arkiv. Math. Astron. Fysik 26A,

42. Ionescu-Tulcea, A., Ionescu-Tulcea, C.: Topics in the theory of lifting. Berlin, Heidelberg, New York: Springer 1970

43. Iske, P. L., Caspers, W. J.: Exact ground states of one-dimensional valence-bond-solid Hamiltonians. Mod. Phys. Lett. B1, 231-237 (1987)

44. Iske, P. L., Caspers, W. J.: Scaling properties of valence-bond-solids with a linear structure. Mod. Phys. Lett. B2, 1223-1233 (1988)

45. Jacobson, N.: Basic Algebra I. San Francisco: Freeman 1974

46. Kennedy, T., Lieb, E. H., Tasaki, H.: A two-dimensional isotropic quantum antiferromagnet with unique disordered ground state. J. Stat. Phys. 53, 383-415 (1988)

47. Kennedy, T., Lieb, E. H., Shastry, B. S.: Existence of Néel order in some spin $1 / 2$ Heisenberg antiferromagnets. J. Stat. Phys. 53, 1019-1030 (1988)

48. Klein, D. J.: Exact ground states for a class of antiferromagnetic Heisenberg models with short range interactions. J. Phys. A15, 661-671 (1982)

49. Klümper, A.: The spectra of $q$-state vertex models and related antiferromagnetic quantum spin chains. J. Phys. A23, 809-823 (1990).

50. Knabe, S.: Energy gaps and elementary excitations for Certain VBS-Quantum Antiferromagnets. J. Stat. Phys. 52, 627-638 (1988)

51. Lieb, E., Schulz, T., Mattis, D.: Two soluble models of an antiferromagnetic chain. Ann. Phys. 16, 407-466 (1961)

52. Lieb, E., Mattis, D.: Ordering Energy Levels of Interacting Spin Systems. J. Math. Phys. 3, 749-751 (1962)

53. Majumdar, C. K., Ghosh, D. K.: On next nearest-neighbor interaction in linear chains, I, II. J. Math. Phys. 10, 1388-1398, and 1399-1402 (1969)

54. Majumdar, C. K.: Antiferromagnetic model with known ground state. J. Phys. C3, 911-915 (1970) 
55. Matsui, T.: Uniqueness of the translationally invariant ground state in quantum spin systems. Commun. Math. Phys. 126, 453-467 (1990)

56. Nachtergaele, B.: Working with Quantum Markov States and their Classical Analogues. In: Accardi, L., von Waldenfelds, W. (eds.). Quantum probability and applications V, Lecture Notes in Mathematics, vol. 442. Berlin, Heidelberg, New York: Springer 1990

57. Schmitt, L., Wittstock, G.: Kernel representation of completely positive Hilbert-Schmidt operators on standard forms. Arch. Math. 38, 453-458 (1982)

58. Spitzer, F.: Markov random fields and Gibbs ensembles. Am. Math. Monthly 78, 142-154 (1971)

59. Stinespring, W. F.: Positive functions on $C^{*}$-algebras. Proc. Am. Math. Soc. 6, 211-216(1955)

60. St $\phi$ rmer, E.: Positive maps of $C^{*}$-algebras. In: Hartkämper, A., Neumann, H. (eds.). Foundations of quantum mechanics and ordered linear spaces. Lecture Notes in Physics, vol. 29, Berlin, Heidelberg, New York: Springer 1974

61. St $\phi$ rmer, E.: Symmetric states of infinite tensor products of $C^{*}$-algebras. J. Funct. Anal. 3, 48-68 (1969)

62. Takesaki, M.: Theory of operator algebras I. Berlin, Heidelberg, New York: Springer 1979

63. Takhtajan, L. A.: The picture of low-lying excitations in the isotropic Heisenberg chain of arbitrary spins. Phys. Lett. 87A, 479-482 (1982)

64. Werner, R. F.: Quantum states with Einstein-Rosen-Podolsky correlations admitting a hidden-variable model. Phys. Rev. A 40, 4277-4281 (1989)

65. Werner, R. F.: Remarks on a quantum state extension problem. Lett. Math. Phys. 19, 319-326 (1990)

Communicated by M. Aizenman 